\title{
Combinatorial proofs in bordered Heegaard Floer homology
}

\author{
BOHUA ZHAN
}

\begin{abstract}
Using bordered Floer theory, we give a combinatorial construction and proof of invariance for the hat version of Heegaard Floer homology. As part of the proof, we also establish combinatorially the invariance of the linear-categorical representation of the strongly based mapping class groupoid given by the same theory.
\end{abstract}

57R58; 57R56

\section{Introduction}

Heegaard Floer homology, introduced by Ozsváth and Szabó [14; 15], gives several kinds of invariants for closed 3-manifolds. The invariants are defined using holomorphic curves, so in general they are not directly computable from their definitions. However, for the hat version of the invariant, denoted $\widehat{\mathrm{HF}}$, there are ways to give combinatorial definitions. There are two steps in this process. First, we want to give to a particular kind of description of a 3-manifold (such as a Heegaard splitting) a description of $\widehat{\mathrm{HF}}$ associated to that 3-manifold. This means that, at least in principle, the invariant can be computed algorithmically for any 3-manifold. Second, we want to give combinatorial proofs for the main properties of $\widehat{\mathrm{HF}}$, beginning with the statement that it depends only on the diffeomorphism class of the 3-manifold, rather than on a particular description of it.

Bordered Floer theory gives a way to extend the hat version of Heegaard Floer homology to 3-manifolds with one or two boundary components. The theory is also defined using holomorphic curves. However, some of the invariants associated to certain simple types of 3-manifolds with boundary have been computed. By breaking an arbitrary closed 3-manifold into simpler pieces, the theory gives a combinatorial description of $\widehat{\mathrm{HF}}$ (see Lipshitz, Ozsváth and Thurston [11]) achieving the first step in the process described above.

In this paper, we give the second step of the process; namely, we prove combinatorially that the construction of $\widehat{\mathrm{HF}}$ given by bordered Floer theory in fact produces an invariant of the 3-manifold. One main result we use is an alternative description of $\widehat{\mathrm{CFA} A}\left(\mathbb{I}_{\mathcal{Z}}\right)$ given by Zhan [21]. This allows us to use a combinatorial construction that is easier to reason about. 
An intermediate statement in the proof, which may be of independent interest, is that bordered Floer theory gives a linear-categorical representation of the strongly based mapping class groupoid, which contains the strongly based mapping class group. By a linear-categorical representation of a group or groupoid, we mean assigning homotopy equivalence classes of bimodules to each element of the group (resp. groupoid), in such a way that composition in the group (resp. groupoid) corresponds to taking an appropriate tensor product of bimodules.

Sarkar and Wang [16] gave the first combinatorial description of $\widehat{\mathrm{HF}}$, giving a systematic way to convert any Heegaard diagram into a nice diagram, in which counting holomorphic curves is combinatorial. Ozsváth, Stipsicz, and Szabó [13] gave the first combinatorial proof of invariance for $\widehat{\mathrm{HF}}$, using another way to convert general Heegaard diagrams into convenient diagrams - a more restricted kind of nice diagram and by studying Heegaard moves on convenient diagrams.

Linear-categorical representations of important groups in topology have also been investigated before. Bordered Floer theory actually gives a family of representations of the strongly based mapping class groupoid. For a given genus $g$, the representations are indexed by an integer $w$, called the weight, between $-g$ and $g$. The representation that is relevant for 3-manifold invariants, and that we will focus on in this paper, corresponds to $w=0$. The cases $w= \pm g$ are trivial. The cases $w= \pm(g-1)$ are described combinatorially by Lipshitz, Ozsváth and Thurston [9], and a combinatorial proof of invariance is given by Siegel [19]. Linear-categorical representations of other groups occurring in topology have also been studied; see the introduction by Khovanov and Thomas [7] for a review and a list of references. One major example is linearcategorical representations of the braid group, studied by, for example, Khovanov and Seidel [6], Seidel and Thomas [18], Cautis and Kamnitzer [3], Seidel and Smith [17], and Khovanov [5].

In Section 2, we review the structure of bordered Floer theory, and describe its combinatorial construction as considered here. In Section 3, we prove some preliminary results on type $D A$ bimodules and our construction of the type $D A$ invariants. Using these results, we prove in Section 4 the intermediate statement on the linear-categorical representation of the strongly based mapping class groupoid. Finally, we complete the proof of invariance for closed 3-manifolds in Section 5.

Acknowledgements I would like to thank Peter Ozsváth for offering the ideas which led to this paper, and to Zoltán Szabó and Robert Lipshitz for many suggestions. I also want to thank the Simons Center for Geometry and Physics for their hospitality while part of this work is done. Finally, I thank the referee for many helpful comments. 


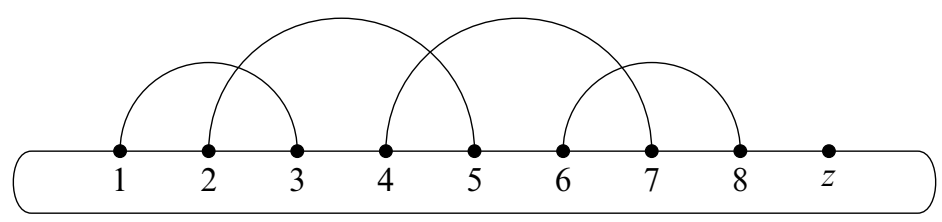

Figure 1: Linear pointed matched circle with $k=2$

\section{Overview of the construction}

In the first part of this section, we briefly review the structure of bordered Floer theory, as is defined analytically in $[10 ; 12]$. In the second part, we describe some of the existing combinatorial constructions given in [11], and then the construction that will be studied in this paper.

\section{A Pointed matched circles and strand algebras}

In bordered Floer theory, the connected, compact, orientable surfaces that serve as boundary components of 3-manifolds are specified using pointed matched circles. A pointed matched circle is a quadruple $\mathcal{Z}=(Z, z, \boldsymbol{a}, M)$, consisting of a circle $Z$, a point $z \in Z$, a set of $4 k$ points $\boldsymbol{a} \subset Z \backslash\{z\}$, and a two-to-one map $M$ from $\boldsymbol{a}$ to $\{1,2, \ldots, 2 k\}$, pairing the points in $\boldsymbol{a}$, that satisfies the following condition: if we thicken the circle $Z$ to an annulus $Z \times[0,1]$ and attach a 1 -handle to the outside boundary $Z \times\{1\}$ of the annulus joining each pair of points in $\boldsymbol{a}$, then the new outside boundary must be a single circle. Given this requirement, we may glue a disk onto that boundary, obtaining a genus $k$ surface $F^{\circ}(\mathcal{Z})$ with one boundary component $Z \times\{0\}$ and a basepoint $z$ on the boundary. We say that the pointed matched circle $\mathcal{Z}$ parametrizes $F^{\circ}(\mathcal{Z})$. Let $-\mathcal{Z}$ be the pointed matched circle obtained by reversing orientation on $\mathcal{Z}$. Then $F^{\circ}(-\mathcal{Z})$ is the orientation reversal of $F^{\circ}(\mathcal{Z})$.

Let $F(\mathcal{Z})$ be the result of filling the boundary of $F^{\circ}(\mathcal{Z})$ with a disk. Then $F(\mathcal{Z})$ is a closed surface of genus $k$, marked with a homotopically trivial circle $Z$ and a basepoint $z \in Z$. We will also say $F(\mathcal{Z})$ is parametrized by $\mathcal{Z}$.

An example of a pointed matched circle for $k=2$ is shown in Figure 1.

To each pointed matched circle $\mathcal{Z}$, bordered Floer theory associates a combinatorially defined DG algebra $\mathcal{A}(\mathcal{Z})$. We refer to the original papers for the description of $\mathcal{A}(\mathcal{Z})$. Here we just fix some notations and terminologies used in this paper. For any generator $a \in \mathcal{A}(\mathcal{Z})$, the multiplicity of $a$, denoted $\operatorname{mult}(a)$, is an element in $H_{1}(Z \backslash\{z\}, \boldsymbol{a})$ recording how many times the strands in $a$ cover each nonbasepoint interval on $Z$. The length of $a$ is the sum of coefficients in mult $(a)$. Equivalently, it is the sum of 
lengths of the strands in $a$. It is clear from the definitions that the algebra $\mathcal{A}(-\mathcal{Z})$ is the opposite algebra of $\mathcal{A}(\mathcal{Z})$. In particular, there is a canonical identification of their generators. For any $a \in \mathcal{A}(\mathcal{Z})$, let $\bar{a}$ denote the corresponding element in $\mathcal{A}(-\mathcal{Z})$. If $i \in \mathcal{A}(\mathcal{Z})$ is an idempotent, let $o(i) \in \mathcal{A}(\mathcal{Z})$ denote the idempotent complementary to $i$. A chord is a single strand on $Z$. For any given chord $\xi$, we define $a(\xi) \in \mathcal{A}(\mathcal{Z})$ to be the sum of all generators that result from adding horizontal strands to $\xi$.

Given a 3-manifold $Y$ with one boundary component $\partial Y$, a parametrization of $\partial Y$ by a pointed matched circle $\mathcal{Z}=(Z, z, \boldsymbol{a}, M)$ is a diffeomorphism $\phi: F(\mathcal{Z}) \rightarrow \partial Y$. This marks $\partial Y$ with a circle and a basepoint on the circle, which by abuse of notation we will also call $Z$ and $z$. Bordered Floer theory associates two invariants to a 3-manifold $Y$ with boundary $\partial Y$ parametrized by $\mathcal{Z}$ :

- A type $A$ invariant $\widehat{\mathrm{CFA}}(Y)_{\mathcal{A}(\mathcal{Z})}$ is a right $A_{\infty}$-module over $\mathcal{A}(\mathcal{Z})$.

- A type $D$ invariant ${ }^{A(-Z)} \widehat{\mathrm{CFD}}(Y)$ is a left type $D$ module over $\mathcal{A}(-\mathcal{Z})$.

They are invariants of $Y$ up to homotopy equivalence of $A_{\infty}$-modules or type $D$ modules. We use the following standard convention in expressing types of actions on the module: each algebra is written on the side it acts on, subscripts indicate $A_{\infty}-$ actions, and superscripts indicate type $D$ actions. These may be omitted when there is no danger of confusion.

These invariants satisfy the following pairing theorem: let $Y_{1}$ and $Y_{2}$ be two 3manifolds with boundaries parametrized by $\mathcal{Z}$ and $-\mathcal{Z}$, respectively. Let $Y=Y_{1} \cup_{\partial} Y_{2}$ be the closed 3-manifold obtained by gluing them along their boundaries (with the gluing map induced by the parametrizations). Then the chain complex $\widehat{\mathrm{CF}}(Y)$, whose homology is $\widehat{\mathrm{HF}}(Y)$, is given by

$$
\widehat{\mathrm{CF}}(Y) \simeq \widehat{\mathrm{CFA}}\left(Y_{1}\right)_{\mathcal{A}(\mathcal{Z})} \otimes^{\mathcal{A}(\mathcal{Z})} \widehat{\mathrm{CFD}}\left(Y_{2}\right)
$$

[10, Theorem 1.3].

The theory extends to 3-manifolds with two boundary components as follows: given $Y$ with two boundary components $\partial_{L} Y$ and $\partial_{R} Y$, fix parametrizations $\phi_{1}: F\left(\mathcal{Z}_{1}\right) \rightarrow \partial_{L} Y$ and $\phi_{2}: F\left(\mathcal{Z}_{2}\right) \rightarrow \partial_{R} Y$. This induces circles $Z_{1}$ and $Z_{2}$ on $\partial_{L} Y$ and $\partial_{R} Y$, and basepoints $z_{1} \in Z_{1}, z_{2} \in Z_{2}$. We further fix a map $\gamma$ from the framed cylinder $\left(S^{1}, z\right) \times[0,1]$ into $Y$, so that $\left(S^{1}, z\right) \times\{0\}$ and $\left(S^{1}, z\right) \times\{1\}$ map to $\left(Z_{1}, z_{1}\right)$ and $\left(Z_{2}, z_{2}\right)$, respectively. We call the totality of the data $\left(Y, \partial_{L} Y, \partial_{R} Y, \phi_{1}, \phi_{2}, \gamma\right)$ a strongly bordered 3-manifold with two boundary components. From now on whenever we mention a 3-manifold $Y$ with two boundary components, we mean a strongly bordered 3-manifold, omitting the other data when they are clear from context. To a 3-manifold $Y$ with two boundary components, bordered Floer theory associates the following invariants: 
- A type $A A$ invariant $\widehat{\mathrm{CFA}}(Y)_{\mathcal{A}\left(\mathcal{Z}_{1}\right), \mathcal{A}\left(\mathcal{Z}_{2}\right)}$ is a right $A_{\infty}$-bimodule over $\mathcal{A}\left(\mathcal{Z}_{1}\right)$ and $\mathcal{A}\left(\mathcal{Z}_{2}\right)$.

- A type $D D$ invariant $\mathcal{A}\left(-\mathcal{Z}_{1}\right), \mathcal{A}\left(-Z_{2}\right) \widehat{C} \widehat{\mathrm{FDD}}(Y)$ is a left type $D$ bimodule over $\mathcal{A}\left(-\mathcal{Z}_{1}\right)$ and $\mathcal{A}\left(-\mathcal{Z}_{2}\right)$.

- A type $D A$ invariant ${ }^{A\left(-Z_{1}\right)} \widehat{\mathrm{CFDA}}(Y)_{\mathcal{A}\left(\mathcal{Z}_{2}\right)}$ is a left type $D$, right $A_{\infty}$-bimodule over $\mathcal{A}\left(-\mathcal{Z}_{1}\right)$ and $\mathcal{A}\left(\mathcal{Z}_{2}\right)$.

- A type $A D$ invariant ${ }^{A\left(-Z_{2}\right)} \widehat{\mathrm{CFAD}}(Y)_{\mathcal{A}\left(\mathcal{Z}_{1}\right)}$ is a right $A_{\infty}$, left type $D$ bimodule over $\mathcal{A}\left(\mathcal{Z}_{1}\right)$ and $\mathcal{A}\left(-\mathcal{Z}_{2}\right)$.

These bimodules satisfy similar pairing theorems, as described in [12, Section 7.1]. The general rule is that box tensor products can be taken between a right $A_{\infty}$-action and a left type $D$ action over the same algebra $\mathcal{A}(\mathcal{Z})$. Taking this box tensor product corresponds to gluing two boundaries parametrized by $\mathcal{Z}$ and $-\mathcal{Z}$.

Following the convention in [21], we will write actions on the various kinds of modules and bimodules as sums of arrows. For example, if the coefficient of $\boldsymbol{y}$ is 1 in $m_{1, i, j}\left(\boldsymbol{x} ; a_{1}, \ldots, a_{i} ; b_{1}, \ldots, b_{j}\right)$, where each $a_{k}, 1 \leq k \leq i$ and $b_{l}, 1 \leq l \leq j$ is a generator of the appropriate algebra, we say there is an arrow

$$
m_{1, i, j}:\left(\boldsymbol{x} ; a_{1}, \ldots, a_{i} ; b_{1}, \ldots, b_{j}\right) \rightarrow \boldsymbol{y} .
$$

Likewise, an arrow in the type $D A$ action is of the form

$$
\delta_{1+i}^{1}:\left(\boldsymbol{x}, a_{1}, \ldots, a_{i}\right) \rightarrow b \otimes \boldsymbol{y},
$$

and an arrow in the type $D D$ action is of the form

$$
\delta^{1}: \boldsymbol{x} \rightarrow a \otimes b \otimes \boldsymbol{y} .
$$

We will also need the concept of duality on bimodules, called opposite structures in [12, Definition 2.2.31, 2.2.53]. For a left type $D D$ bimodule ${ }^{A, B} M$, its dual $\bar{M}^{A, B}$ is the type $D D$ bimodule over the same generators, where each arrow $\delta_{M}^{1}: \boldsymbol{x} \rightarrow a_{1} \otimes a_{2} \otimes \boldsymbol{y}$ in the type $D D$ action of $A, B M$ corresponds to an arrow $\delta_{\bar{M}}^{1}: \boldsymbol{y} \rightarrow a_{1} \otimes a_{2} \otimes \boldsymbol{x}$ in the type $D D$ action of $\bar{M}^{A, B}$. In this way the left actions by $A$ and $B$ (equivalently left actions by $A^{\text {opp }}$ and $B^{\text {opp }}$ ) become right actions. Thus, we will also write the dual as $A^{\text {opp }}, B^{\text {opp }} \bar{M}$. Similarly, we can define duals on type $D A$ and type $A A$ bimodules. The dual commutes with box tensor product, that is,

$$
\overline{M_{A} \otimes^{A} N}=\bar{N}^{A} \otimes_{A} \bar{M}=\bar{M}_{A^{\text {opp }}} \otimes^{A^{\mathrm{opp}}} \bar{N},
$$

where $M$ and $N$ may have additional actions. 


\section{B Gradings on bordered invariants}

In this section we give a brief overview of gradings on the bordered invariants. For details, see [10, Chapter 10] and [12, Section 6.5].

We begin with gradings on the DG algebra $\mathcal{A}(\mathcal{Z})$. There are two kinds of gradings: one by a larger group $G^{\prime}(\mathcal{Z})$, and a refined grading by a smaller group $G(\mathcal{Z})$. Both $G(\mathcal{Z})$ and $G^{\prime}(\mathcal{Z})$ are noncommutative, equipped with a distinguished central element $\lambda$.

An element of $G^{\prime}(\mathcal{Z})$ is specified by a pair $(k, \alpha)$, where $k \in \frac{1}{2} \mathbb{Z}$ and $\alpha \in H_{1}\left(Z^{\prime}, \boldsymbol{a}\right)$. With points of $\boldsymbol{a}$ labeled $1, \ldots, 4 k$, we can write $\alpha$ as a sequence of integers $\alpha_{i}, 1 \leq i \leq$ $4 k-1$, where $\alpha_{i}$ is the multiplicity of $\alpha$ at the interval $[i, i+1]$. Then multiplication on $G^{\prime}(\mathcal{Z})$ is defined by

$$
(k, \alpha) \cdot(l, \beta)=(k+l+L(\alpha, \beta), \alpha+\beta),
$$

where

$$
L(\alpha, \beta)=\sum_{i=1}^{4 k-2} \frac{1}{2}\left(\alpha_{i} \beta_{i+1}-\alpha_{i+1} \beta_{i}\right) .
$$

Actually, the grading lies in an index 2 subgroup of $G^{\prime}(\mathcal{Z})$, but we will not make use of this here.

For later use, we define an antihomomorphism

$$
R: G^{\prime}(\mathcal{Z}) \rightarrow G^{\prime}(-\mathcal{Z})
$$

given by

$$
R\left(k, \alpha_{1}, \ldots, \alpha_{4 k-1}\right)=\left(k,-\alpha_{4 k-1}, \ldots,-\alpha_{1}\right) .
$$

To define the grading of a generator of $\mathcal{A}(\mathcal{Z})$, we first define a map

$$
m: H_{1}\left(Z^{\prime}, \boldsymbol{a}\right) \times H_{0}(\boldsymbol{a}) \rightarrow \frac{1}{2} \mathbb{Z} .
$$

For an interval $\alpha$ (with orientation from $Z$ ) and a point $p$, let $m(\alpha, p)$ equal 1 if $p$ is in the interior of $\alpha, \frac{1}{2}$ if $p$ is on the boundary, and 0 otherwise. This is then extended bilinearly to all of $H_{1}\left(Z^{\prime}, \boldsymbol{a}\right) \times H_{0}(\boldsymbol{a})$ to define $m$.

Given a generator $a \in \mathcal{A}(\mathcal{Z})$, let $\rho$ be the nonhorizontal strands of $a$. Let $\operatorname{inv}(\rho)$ be the number of inversions in $\rho, S \in H_{0}(\boldsymbol{a})$ be the starting points of $\boldsymbol{\rho}$, and $[a] \in H_{1}\left(Z^{\prime}, \boldsymbol{a}\right)$ be the multiplicity of $a$. Then

$$
\operatorname{gr}^{\prime}(a)=(\operatorname{inv}(\rho)-m([a], S),[a]) .
$$

Next, we consider relative gradings on the type $D$ invariant. Fix a bordered Heegaard diagram $\mathcal{H}$. Let $\boldsymbol{x}, \boldsymbol{y}$ be generators and $B \in \pi_{2}(\boldsymbol{x}, \boldsymbol{y})$, define $g^{\prime}(B) \in G^{\prime}(\mathcal{Z})$ as

$$
g^{\prime}(B)=\left(-e(B)-n_{\boldsymbol{x}}(B)-n_{\boldsymbol{y}}(B), \partial^{\partial}(B)\right) .
$$


Here $e(B)$ is the Euler measure of $B$, and $n_{x}(B), n_{y}(B)$ are multiplicities of $B$ at $\boldsymbol{x}, \boldsymbol{y}$ (each corner around $\boldsymbol{x}$ or $\boldsymbol{y}$ counts as multiplicity $\frac{1}{4}$ ), and $\partial^{\partial}(B)$ is the boundary of $B$ on $H_{1}\left(Z^{\prime}, \boldsymbol{a}\right)$.

There is a grading set for each $\operatorname{spin}^{\mathrm{c}}$ class on $\mathcal{H}$ (in most bordered cases we consider here, there is just one $\operatorname{spin}^{\mathrm{c}}$ class). The grading set $S_{D}^{\prime}(\mathcal{H}, \mathfrak{s})$ for the Heegaard diagram $\mathcal{H}$ and $\operatorname{spin}^{\mathrm{c}}$ class $\mathfrak{s}$ is defined as follows: choose a base generator $\boldsymbol{x}_{0}$ with $\operatorname{spin}^{\mathrm{c}}$ class $\mathfrak{s}$. Let $P^{\prime}\left(x_{0}\right)$ be the set of $g^{\prime}(P)$ for all $P \in \pi_{2}\left(x_{0}, x_{0}\right)$ (the domains in $\pi_{2}\left(x_{0}, x_{0}\right)$ are called periodic domains). Then

$$
S_{D}^{\prime}(\mathcal{H}, \mathfrak{s})=G^{\prime}(-\mathcal{Z}) / R\left(P^{\prime}\left(x_{0}\right)\right) .
$$

This grading set has an obvious left action by $G^{\prime}(\mathcal{Z})$. For another generator $\boldsymbol{x}$ in the same $\operatorname{spin}^{\mathrm{c}}$ class, choose a domain $B_{0} \in \pi_{2}\left(\boldsymbol{x}_{0}, \boldsymbol{x}\right)$, and set

$$
\operatorname{gr}^{\prime}(\boldsymbol{x})=R\left(g^{\prime}\left(B_{0}\right)\right) \cdot R\left(P^{\prime}\left(\boldsymbol{x}_{0}\right)\right) .
$$

The type $D$ action respects this relative grading in the sense that, for each arrow $\delta^{1}: \boldsymbol{x} \rightarrow a \otimes \boldsymbol{y}$ in the action, we have

$$
\lambda^{-1} \operatorname{gr}^{\prime}(\boldsymbol{x})=\operatorname{gr}^{\prime}(a) \operatorname{gr}^{\prime}(\boldsymbol{y}) .
$$

Relative gradings on type $A$ invariants are similar. The grading set is

$$
S_{A}^{\prime}(\mathcal{H}, \mathfrak{s})=P^{\prime}\left(\boldsymbol{x}_{0}\right) \backslash G^{\prime}(\mathcal{Z}) .
$$

This carries a natural right action by $G^{\prime}(\mathcal{Z})$. For any generator $\boldsymbol{x}$ in the $\operatorname{spin}^{\mathrm{c}}$ class $\mathfrak{s}$, choose a domain $B_{0} \in \pi_{2}\left(x_{0}, \boldsymbol{x}\right)$ and set

$$
\operatorname{gr}^{\prime}(x)=P^{\prime}\left(x_{0}\right) \cdot g^{\prime}\left(B_{0}\right) .
$$

The $A_{\infty}$-action respects the relative grading in the sense that, for each arrow

we have

$$
m_{1, k}:\left(\boldsymbol{x} ; a_{1}, \ldots, a_{k}\right) \rightarrow \boldsymbol{y}
$$

$$
\lambda^{k-1} \operatorname{gr}^{\prime}(\boldsymbol{x}) \operatorname{gr}^{\prime}\left(a_{1}\right) \cdots \operatorname{gr}^{\prime}\left(a_{k}\right)=\operatorname{gr}^{\prime}(\boldsymbol{y}) .
$$

Gradings on bimodules are defined similarly. In particular, a domain in a Heegaard diagram with two boundary components parametrized by $\mathcal{Z}_{1}$ and $\mathcal{Z}_{2}$ gives rise to an element of

$$
G^{\prime}\left(\mathcal{Z}_{1}\right) \times_{\lambda} G^{\prime}\left(\mathcal{Z}_{2}\right)=G^{\prime}\left(\mathcal{Z}_{1}\right) \times G^{\prime}\left(\mathcal{Z}_{2}\right) /\left(\lambda_{1}=\lambda_{2}\right) .
$$

The grading set is a certain coset of $G^{\prime}\left(\mathcal{Z}_{1}\right) \times_{\lambda} G^{\prime}\left(\mathcal{Z}_{2}\right)$.

Now we briefly discuss refined gradings, which contain essentially the same information, but are cleaner to work with theoretically. 
The group $G(\mathcal{Z})$ can be considered as a subgroup of $G^{\prime}(\mathcal{Z})$, generated by $\lambda$ and elements of the form $(0,[p, q])$, where $p, q$ is a pair of matched points, and $[p, q]$ denotes the interval in $H_{1}\left(Z^{\prime}, \boldsymbol{a}\right)$ between $p$ and $q$. An element $(k, \alpha)$ of $G^{\prime}(\mathcal{Z})$ is in $G(\mathcal{Z})$ if and only if $M_{*}(\partial \alpha)=0$, where $\partial: H_{1}\left(Z^{\prime}, \boldsymbol{a}\right) \rightarrow H_{0}(\boldsymbol{a})$ is the boundary operator and $M_{*}: H_{0}(\boldsymbol{a}) \rightarrow \mathbb{Z}^{2 k}$ is a map sending each matched pair of points to the same basis element of $\mathbb{Z}^{2 k}$.

To construct the refined grading on $\mathcal{A}(\mathcal{Z})$, we first choose a base idempotent $s_{0}$ in $\mathcal{A}(\mathcal{Z})$. Then for every idempotent $s$, choose a grading element $\psi(s)=(k, \alpha) \in G^{\prime}(\mathcal{Z})$ such that $M_{*}(\partial \alpha)=s-s_{0}$. For an algebra element $a$ with left idempotent $s$ and right idempotent $t$, we set

$$
\operatorname{gr}(a)=\psi(s) \operatorname{gr}^{\prime}(a) \psi(t)^{-1} .
$$

It is easy to check that this element lies in $G(\mathcal{Z})$ and that the two conditions on the grading are satisfied.

Similarly, we can refine the grading on the bordered invariants to use $G(\mathcal{Z})$ rather than $G^{\prime}(\mathcal{Z})$. We will omit the details here.

We will not perform any detailed grading computations in this paper, but will simply note that all such computations can be done combinatorially from the Heegaard diagram. For a module (or bimodule) $M$ of any type, grading imposes a constraint on what kind of arrows can appear in the $A_{\infty}$ or type $D$ action on $M$. One such constraint is this: if a domain in a Heegaard diagram with two boundary components touches the two boundaries at intervals $i$ and $i^{\prime}$, respectively, then for each arrow in the algebra action of a bimodule corresponding to that Heegaard diagram, its multiplicities at $i$ and at $i^{\prime}$ must be the same. Such constraints are crucial in establishing uniqueness properties of bimodule invariants, to be discussed in the following sections.

\section{C The strongly based mapping class groupoid}

An important class of 3-manifolds with two boundary components is the mapping cylinders of surface diffeomorphisms. Gluing with these 3-manifolds can be considered as changing the parametrization on the boundary of a bordered 3-manifold.

The strongly based mapping class groupoid of genus $g$ is a category whose objects are pointed matched circles with $4 g$ points. Each object $\mathcal{Z}$ corresponds to a surface $F^{\circ}(\mathcal{Z})$ of genus $g$, with standard parametrization by $\mathcal{Z}$. The morphisms from $\mathcal{Z}_{1}$ to $\mathcal{Z}_{2}$ in the category are isotopy classes of diffeomorphisms $\phi: F^{\circ}\left(\mathcal{Z}_{1}\right) \rightarrow F^{\circ}\left(\mathcal{Z}_{2}\right)$, sending the basepoint $z_{1} \in F^{\circ}\left(\mathcal{Z}_{1}\right)$ to the basepoint $z_{2} \in F^{\circ}\left(\mathcal{Z}_{2}\right)$. Identity and composition in the category correspond to the identity diffeomorphism and composition of diffeomorphisms, respectively. 
If we fix a pointed matched circle $\mathcal{Z}$ and only consider morphisms from $\mathcal{Z}$ to itself, we obtain the strongly based mapping class group of $F_{g, 1}$ (where $F_{g, 1}$ denotes a genus $g$ surface with one circle boundary). This is simply the group of isotopy classes of boundary-preserving self-diffeomorphisms of $F_{g, 1}$.

Given a diffeomorphism $\phi: F^{\circ}\left(\mathcal{Z}_{1}\right) \rightarrow F^{\circ}\left(\mathcal{Z}_{2}\right)$, we can construct its mapping cylinder $Y(\phi)=F\left(\mathcal{Z}_{2}\right) \times[0,1]$ as a strongly bordered 3-manifold with two boundary components, parametrized by $-\mathcal{Z}_{1}$ and $\mathcal{Z}_{2}$. The left boundary $\partial_{L} Y(\phi)=F\left(\mathcal{Z}_{2}\right) \times\{0\}$ is parametrized by the induced map $\phi_{*}:-F\left(\mathcal{Z}_{1}\right) \rightarrow-F\left(\mathcal{Z}_{2}\right)$ (reverse orientation and extend over the disk filling the boundary), while the right boundary is parametrized by the identity map on $F\left(\mathcal{Z}_{2}\right)$. The map $\gamma:\left(S^{1}, z\right) \times[0,1] \rightarrow Y(\phi)$ is simply the inclusion $(Z, z) \times[0,1] \rightarrow F\left(\mathcal{Z}_{2}\right) \times[0,1]$.

This establishes a one-to-one correspondence between strongly bordered 3-manifolds that are topologically $F_{g} \times[0,1]$, and morphisms in the strongly based mapping class groupoid with genus $g$. For a morphism $\phi: F^{\circ}\left(\mathcal{Z}_{1}\right) \rightarrow F^{\circ}\left(\mathcal{Z}_{2}\right)$, we write $\widehat{\mathrm{CFAA}}(\phi)_{\mathcal{A}\left(-\mathcal{Z}_{1}\right), \mathcal{A}\left(\mathcal{Z}_{2}\right)}$ to denote the type $A A$ invariant $\widehat{\mathrm{CFA} A}(Y(\phi))_{\mathcal{A}\left(-\mathcal{Z}_{1}\right), \mathcal{A}\left(\mathcal{Z}_{2}\right)}$ associated to the mapping cylinder of $\phi$. Likewise, we use notations $\mathcal{A}\left(\mathcal{Z}_{1}\right) \widehat{C F D A}(\phi)_{\mathcal{A}\left(\mathcal{Z}_{2}\right)}$ and $\mathcal{A}\left(\mathcal{Z}_{1}\right), \mathcal{A}\left(-\mathcal{Z}_{2}\right) \widehat{\mathrm{CDD}}(\phi)$ for the other invariants corresponding to $Y(\phi)$.

For future reference, we write down the pairing theorems involving $D A$ invariants. For morphisms $\phi_{1}: F^{\circ}\left(\mathcal{Z}_{1}\right) \rightarrow F^{\circ}\left(\mathcal{Z}_{2}\right)$ and $\phi_{2}: F^{\circ}\left(\mathcal{Z}_{2}\right) \rightarrow F^{\circ}\left(\mathcal{Z}_{3}\right)$, the $D A$ invariant for $\phi_{2} \circ \phi_{1}: F^{\circ}\left(\mathcal{Z}_{1}\right) \rightarrow F^{\circ}\left(\mathcal{Z}_{3}\right)$ is given by

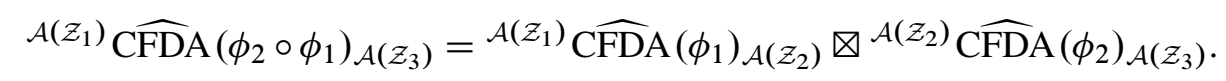

For a morphism $\phi: F^{\circ}\left(\mathcal{Z}_{1}\right) \rightarrow F^{\circ}\left(\mathcal{Z}_{2}\right)$ and a 3 -manifold $Y$ with boundary parametrized by $\psi: F\left(-\mathcal{Z}_{2}\right) \rightarrow \partial Y$, let $Y^{\prime}$ be the same manifold with boundary parametrized by $\psi \circ \phi_{*}: F\left(-\mathcal{Z}_{1}\right) \rightarrow \partial Y$, then

$$
\mathcal{A}\left(\mathcal{Z}_{1}\right) \widehat{\mathrm{CFD}}\left(Y^{\prime}\right)=\mathcal{A}\left(\mathcal{Z}_{1}\right) \widehat{\mathrm{CFDA}}(\phi)_{\mathcal{A}\left(\mathcal{Z}_{2}\right)} \otimes^{\mathcal{A}\left(\mathcal{Z}_{2}\right)} \widehat{\mathrm{CFD}}(Y) .
$$

\section{D Invariants of the identity diffeomorphism}

Let $\mathbb{I}_{\mathcal{Z}}$ be the identity morphism $F^{\circ}(\mathcal{Z}) \rightarrow F^{\circ}(\mathcal{Z})$. All bimodule invariants associated to $\mathbb{I}_{\mathcal{Z}}$ have special significance in the theory. First, it can be shown [12, Section 8.1] that

$$
\widehat{\mathrm{CPDA}}\left(\mathbb{I}_{\mathcal{Z}}\right) \simeq{ }^{\mathcal{A}(\mathcal{Z})} \mathbb{I}_{\mathcal{A}(\mathcal{Z})},
$$

where the latter denotes the identity type $D A$ bimodule over $\mathcal{A}(\mathcal{Z})$. This is the bimodule generated over $\mathbb{F}_{2}$ by idempotents of $\mathcal{A}(\mathcal{Z})$, and with the algebra action given by

$$
\delta_{2}^{1}(i, a)=a \otimes j
$$


for any generator $a \in \mathcal{A}(\mathcal{Z})$, where $i$ and $j$ are the left and right idempotents of $a$.

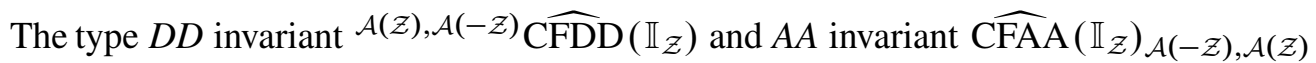
relate the type $A$ and type $D$ invariants through taking the tensor product. For any 3-manifold $Y$ with one boundary component parametrized by $\mathcal{Z}$, the relations are

$$
\begin{aligned}
& \widehat{\mathrm{CFA}}(Y)_{\mathcal{A}(\mathcal{Z})}=\widehat{\mathrm{CFA}}\left(\mathbb{I}_{\mathcal{Z}}\right)_{\mathcal{A}(-\mathcal{Z}), \mathcal{A}(\mathcal{Z})} \otimes^{\mathcal{A}(-\mathcal{Z})} \widehat{\mathrm{CFD}}(Y), \\
& \mathcal{A}(-\mathcal{Z}) \widehat{\mathrm{CFD}}(Y)=\widehat{\mathrm{CFA}}(Y)_{\mathcal{A}(\mathcal{Z})} \mathbb{A}^{\mathcal{A}(\mathcal{Z}), \mathcal{A}(-\mathcal{Z})} \widehat{\mathrm{CFDD}}\left(\mathbb{I}_{\mathcal{Z}}\right) \text {. }
\end{aligned}
$$

One implication is that $\widehat{\mathrm{CFD}}(Y)$ and $\widehat{\mathrm{CFA}}(Y)$ contain the same information about $Y$. Likewise, there are relations among the bimodule invariants, showing that all bimodule invariants also contain the same information. For any 3-manifold $Y$ with two boundary components parametrized by $\mathcal{Z}_{1}$ and $\mathcal{Z}_{2}$, we have

$$
\begin{aligned}
& \mathcal{A}\left(-\mathcal{Z}_{1}\right) \widehat{\mathrm{CFDA}}(Y)_{\mathcal{A}\left(\mathcal{Z}_{2}\right)} \\
& =\widehat{\mathrm{CFA}} \mathrm{A}\left(\mathbb{I}_{\mathcal{Z}_{2}}\right)_{\mathcal{A}\left(-\mathcal{Z}_{2}\right), \mathcal{A}\left(\mathcal{Z}_{2}\right)} \otimes_{\mathcal{A}\left(-\mathcal{Z}_{2}\right)} \mathcal{A ( - \mathcal { Z } _ { 1 } ) , \mathcal { A } ( - \mathcal { Z } _ { 2 } )} \widehat{\mathrm{CFDD}}(Y),
\end{aligned}
$$

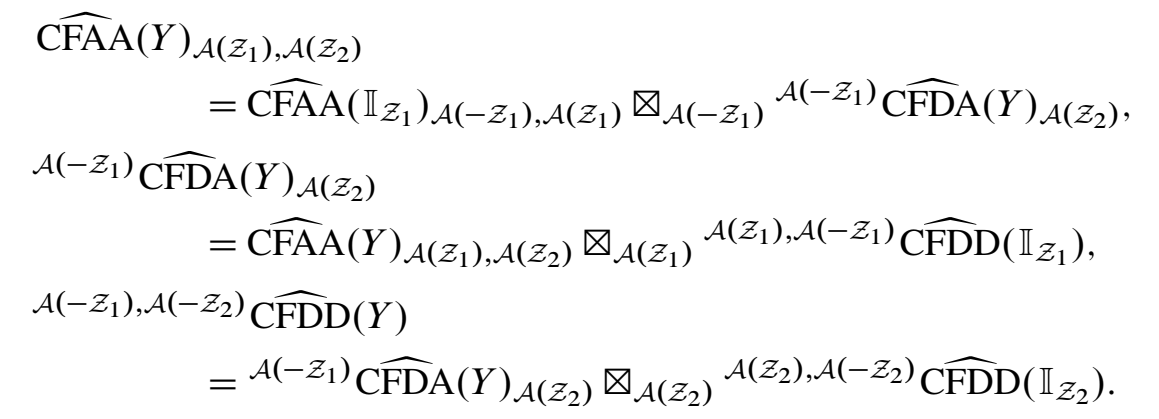

The above equations are special cases of the pairing theorems, where one of the bordered 3 -manifolds is a cylinder with trivial parametrization. They indicate the importance of finding combinatorial descriptions of $\widehat{\operatorname{CFDD}}\left(\mathbb{I}_{\mathcal{Z}}\right)$ and $\widehat{\mathrm{CFA} A}\left(\mathbb{I}_{\mathcal{Z}}\right)$, which we now consider.

First, we describe the combinatorial model $\widehat{\mathcal{D D}}\left(\mathbb{I}_{\mathcal{Z}}\right)$ of $\mathcal{A}(\mathcal{Z}), \mathcal{A}(-\mathcal{Z}) \widehat{\mathrm{CFDD}}\left(\mathbb{I}_{\mathcal{Z}}\right)$, given in [11, Theorem 1]. It is generated over $\mathbb{F}_{2}$ by the set of pairs of complementary idempotents $i \otimes i^{\prime}$, with $i \in \mathcal{A}(\mathcal{Z})$ and $i^{\prime}=\overline{o(i)} \in \mathcal{A}(-\mathcal{Z})$. The type $D D$ action is given by

$$
\delta^{1}\left(i \otimes i^{\prime}\right)=\sum_{\begin{array}{c}
\xi \in \mathcal{C} \\
i a(\xi)=a(\xi) j \\
i^{\prime} a(\xi)
\end{array}=\overline{a(\xi)} j^{\prime}}(a(\xi) \otimes \overline{a(\xi)}) \otimes\left(j \otimes j^{\prime}\right)
$$


where $\mathcal{C}$ is the set of chords on $\mathcal{Z}$ whose two endpoints are not matched. Intuitively, the arrows in the type $D D$ action are exactly those whose two algebra outputs both contain exactly one chord connecting two unpaired points, and covering corresponding intervals in $\mathcal{A}(\mathcal{Z})$ and $\mathcal{A}(-\mathcal{Z})$.

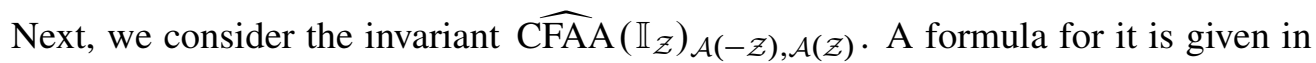
[12, Proposition 9.2] as follows (here we simplify $\mathcal{A}(\mathcal{Z})$ to $\mathcal{A}$ and $\mathcal{A}(-\mathcal{Z})$ to $\mathcal{A}^{\prime}$ ):

$$
\begin{aligned}
& \widehat{\mathrm{CFA} A}\left(\mathbb{I}_{\mathcal{Z}}\right)_{\mathcal{A}^{\prime}, \mathcal{A}}=\operatorname{Mor}^{\mathcal{A}}\left({ }_{\mathcal{A}^{\prime}} \mathcal{A}_{\mathcal{A}^{\prime}}^{\prime} \otimes_{\mathcal{A}^{\prime}}{ }^{\mathcal{A}, \mathcal{A}^{\prime}} \widehat{\operatorname{CFDD}}\left(\mathbb{I}_{\mathcal{Z}}\right),{ }^{\mathcal{A}} \mathbb{I}_{\mathcal{A}}\right)
\end{aligned}
$$

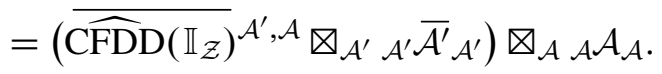

This bimodule has a large number of generators, making it difficult to use for the computations needed in this paper. The main result of [21] is to describe a bimodule $\widehat{\mathcal{A A}}\left(\mathbb{I}_{\mathcal{Z}}\right)$ homotopy equivalent to this (and hence is also a combinatorial model of $\left.\widehat{\mathrm{CFA} A}\left(\mathbb{I}_{\mathcal{Z}}\right)\right)$, but with a minimal number of generators. The bimodule $\widehat{\mathcal{A A}}\left(\mathbb{I}_{\mathcal{Z}}\right)$ is generated over $\mathbb{F}_{2}$ by the set of pairs of complementary idempotents, but with much more complex $A_{\infty}$-bimodule actions. We will briefly review this construction in Section 3C.

One of the pairing theorems imply the following relation among the combinatorial models for $\mathbb{I}_{\mathcal{Z}}$ :

$$
{ }^{\mathcal{A}(\mathcal{Z})} \mathbb{I}_{\mathcal{A}(\mathcal{Z})} \simeq \widehat{\mathcal{A A}}\left(\mathbb{I}_{\mathcal{Z}}\right)_{\mathcal{A}(-\mathcal{Z}), \mathcal{A}(\mathcal{Z})} \otimes_{\mathcal{A}(-\mathcal{Z})} \mathcal{A ( \mathcal { Z } ) , \mathcal { A } ( - \mathcal { Z } )} \widehat{\mathcal{D D}}\left(\mathbb{I}_{\mathcal{Z}}\right)
$$

The two sides are not equal but only homotopy equivalent. This homotopy equivalence is proven combinatorially as Corollary 3.10 in Section 3D.

\section{E Invariants of arcslides}

The strongly based mapping class groupoid is generated by a particularly simple class of morphisms called arcslides. We will now review their definitions and the invariants associated to them. The relations among arcslides will be described at the beginning of Section 3.

Given a pointed matched circle $\mathcal{Z}_{1}$, and two matched pairs of points $B=\left(b_{1}, b_{2}\right)$ and $C=\left(c_{1}, c_{2}\right)$ in $\boldsymbol{a} \subset Z_{1}$, such that $b_{1}$ and $c_{1}$ are adjacent in $\boldsymbol{a}$, an arcslide of $b_{1}$ over $c_{1}$ moves $b_{1}$ to be adjacent to $c_{2}$, on the side opposite to its original position with respect to $c_{1}$. This results in a new pointed matched circle $\mathcal{Z}_{2}$. Such a move corresponds to a certain diffeomorphism $F^{\circ}\left(\mathcal{Z}_{1}\right) \rightarrow F^{\circ}\left(\mathcal{Z}_{2}\right)$, which we will also call an arcslide. See Figure 2 for two examples of arcslides. The first example is an overslide meaning $b_{1}$ is outside the interval $\left[c_{1}, c_{2}\right]$. The second example is an underslide meaning $b_{1}$ is inside that interval. 


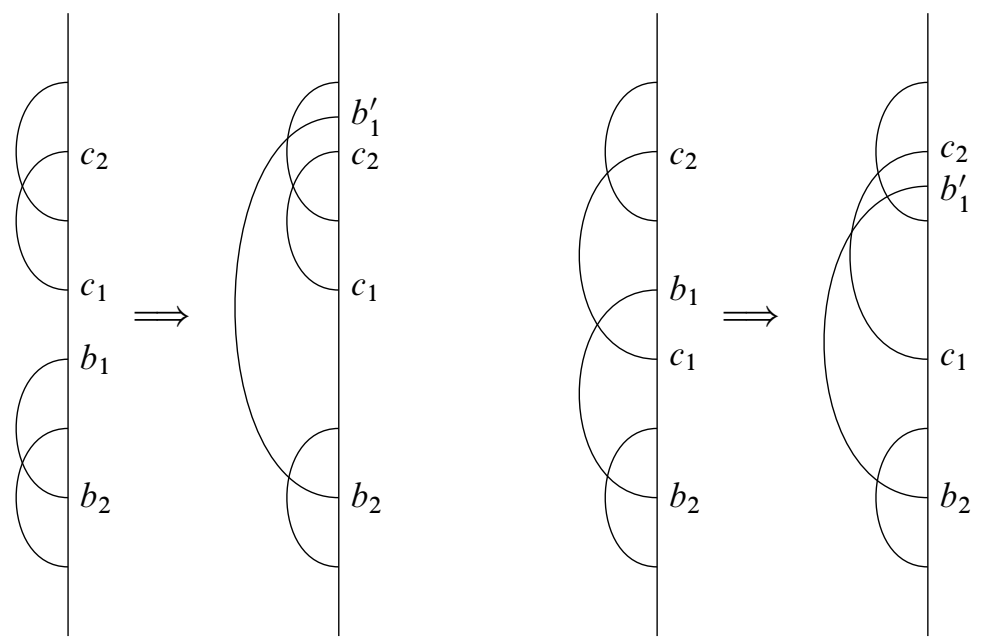

Figure 2: Two examples of arcslides
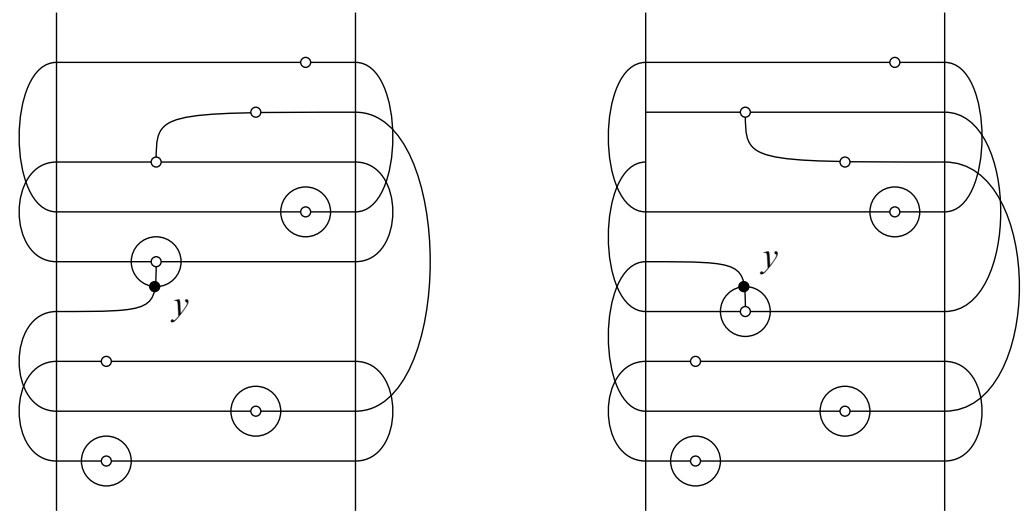

Figure 3: Examples of Heegaard diagrams of arcslides

Given an arcslide $\tau: F^{\circ}\left(\mathcal{Z}_{1}\right) \rightarrow F^{\circ}\left(\mathcal{Z}_{2}\right)$, the invariant $\widehat{\mathrm{CDD}}(\tau)$ is a left type $D D$ bimodule over $A\left(\mathcal{Z}_{1}\right)$ and $A\left(-\mathcal{Z}_{2}\right)$. Constructing a combinatorial model of this bimodule, denoted $\widehat{\mathcal{D D}}(\tau)$, is the main subject of [11]. This model is computed from a standard Heegaard diagram for the mapping cylinder $Y(\tau)$. For the two arcslides in Figure 2, these standard Heegaard diagrams are shown in Figure 3. The tiny circles in the diagrams are 1-handle attachment points, paired according to their vertical positions. The larger circles are $\beta$ circles, and all other arcs inside the boundary are $\alpha$-arcs. Later on, we will draw more schematic versions of these diagrams, omitting some of the $\beta$ circles and attaching points of 1 -handles. 
Each generator of $\widehat{\mathcal{D D}}(\tau)$ corresponds to a $g$-tuple of intersection points between $\alpha$ and $\beta$ curves, where each $\beta$ circle contains exactly one point, and each $\alpha$-arc contains at most one point. The (type $D$ ) idempotent of the generator specifies which pairs of $\alpha$-arcs are not occupied by the generator. For the standard Heegaard diagram of arcslides, a generator is uniquely specified by its idempotents on the two sides.

There is an obvious identification between pairs of points on the two sides, using which we can talk about two idempotents on different sides being complementary, etc. There are two types of generators in $\widehat{\mathcal{D D}}(\tau)$. A generator of type $X$ has complementary idempotents, and a generator of type $Y$ has idempotents that are complementary except for both containing the $C$ pair and neither containing the $B$ pair. The type $X$ generators are those that do not occupy the intersection point $y$ in Figure 3, while the type $Y$ generators do.

The type $D D$ action on the bimodule can be described as follows: given a pointed matched circle $\mathcal{Z}$, let $C(\mathcal{Z})$ denote the collection of sets of chords in $\mathcal{Z}$. For some $\xi \in C(\mathcal{Z})$, let $a(\xi) \in \mathcal{A}(\mathcal{Z})$ denote the sum of all generators of $\mathcal{A}(\mathcal{Z})$ produced by adding horizontal strands to $\xi$ (this definition extends the case where $\xi$ is a chord). For any arcslide $\tau: F^{\circ}\left(\mathcal{Z}_{1}\right) \rightarrow F^{\circ}\left(\mathcal{Z}_{2}\right)$, there is a collection of pairs $C_{\tau} \subseteq\left\{\left(\xi_{i}, \eta_{i}\right) \mid \xi_{i} \in\right.$ $\left.C\left(\mathcal{Z}_{1}\right), \eta_{i} \in C\left(\mathcal{Z}_{2}\right)\right\}$ such that

$$
\delta^{1}\left(i \otimes i^{\prime}\right)=\sum_{\begin{array}{c}
\left(\xi_{k}, \eta_{k}\right) \in C_{\tau} \\
i a\left(\xi_{k}\right)=a\left(\xi_{k}\right) j \\
i^{\prime} \overline{a\left(\eta_{k}\right)}=\overline{a\left(\eta_{k}\right)} j^{\prime} \\
j \otimes j^{\prime} \text { is a generator }
\end{array}}\left(a\left(\xi_{k}\right) \otimes \overline{a\left(\eta_{k}\right)}\right) \otimes\left(j \otimes j^{\prime}\right),
$$

where generators are represented by their type $D$ idempotents.

Intuitively, there is a term in the type $D D$ action whenever the idempotent agrees, and the moving strands part of the algebra coefficients match one of the fixed patterns. Depending on whether the arcslide is an underslide or an overslide, there are six or eight types of elements in $C_{\tau}$. See [11, Figures 21 and 28] for diagrams of these patterns. Note that not all pairs in Figure 28 are actually in $C_{\tau}$ : there is an additional choice involved. In the following computations we will only use some of the simpler pairs, involving algebra elements that have small total length. In particular we will not need to consider any pair where a choice is necessary.

The following properties of $\widehat{\mathcal{D D}}(\tau)$ can be directly verified for the above description:

Relation with Heegaard diagram Every arrow in the type $D D$ action comes from a domain in the Heegaard diagram. For a Heegaard diagram $\mathcal{H}$, write $\boldsymbol{\alpha}$ and $\boldsymbol{\beta}$ to denote the union of $\alpha$ and $\beta$ curves, respectively. A domain in $\mathcal{H}$ is a nonnegative 
integral linear combination of connected components of $\mathcal{H} \backslash\{\boldsymbol{\alpha}, \boldsymbol{\beta}\}$. Each arrow $\boldsymbol{x} \rightarrow a_{1} \otimes a_{2} \otimes \boldsymbol{y}$ in the type $D D$ action comes from a domain $B$, such that $a_{1}$ and $a_{2}$ have multiplicities equal to the multiplicities of $B$ at the corresponding boundaries. Moreover, let $\partial^{\alpha} B$ be the part of the boundary of $B$ on the $\alpha$ curves, and let $\partial\left(\partial^{\alpha} B\right)$ be the part of the boundary of $\partial^{\alpha} B$ in the interior of the diagram, as a signed sum of intersection points, then $\partial\left(\partial^{\alpha} B\right)=\boldsymbol{x}-\boldsymbol{y}$. Intuitively, the $\alpha$-boundaries of $B$ start at points of $\boldsymbol{x}$ and end at points of $\boldsymbol{y}$, and vice versa for the $\beta$-boundary.

Grading There is a refined grading on the generators of $\widehat{\mathcal{D D}}(\tau)$ to a particular grading set $S_{\tau}$, which has left-right actions by $G\left(\mathcal{Z}_{1}\right)$ and $G\left(\mathcal{Z}_{2}\right)$. Both actions are free and transitive, which means $S_{\tau}$ induces a group isomorphism $G\left(\mathcal{Z}_{1}\right) \rightarrow G\left(\mathcal{Z}_{2}\right)$. This group isomorphism is an invariant of $\tau$, up to composing by inner automorphisms of $G\left(\mathcal{Z}_{1}\right)$ and $G\left(\mathcal{Z}_{2}\right)$. In other words, $\tau$ induces an element in the set of outer isomorphisms $\operatorname{Out}\left(G\left(\mathcal{Z}_{1}\right), G\left(\mathcal{Z}_{2}\right)\right)$. In fact, this outer isomorphism corresponds to the actions of $\tau$ on the homology of the surface; see [11, Section 6.2] for details.

Stabilization Given arcslide $\tau: F^{\circ}\left(\mathcal{Z}_{1}\right) \rightarrow F^{\circ}\left(\mathcal{Z}_{2}\right)$, let $\stackrel{\circ}{\mathcal{Z}}_{1}=\mathcal{Z}_{1} \# \mathcal{Z}^{1}$ and $\stackrel{\circ}{\mathcal{Z}}_{2}=$ $\mathcal{Z}_{2} \# \mathcal{Z}^{1}$, where $\mathcal{Z}^{1}$ is the genus 1 pointed matched circle, and \# denotes connect sum on pointed matched circles. Let $\stackrel{\circ}{\tau}: F\left(\stackrel{\circ}{\mathcal{Z}}_{1}\right) \rightarrow F\left(\stackrel{\circ}{\mathcal{Z}}_{2}\right)$ be the arcslide acting as identity on the new handle, and as $\tau$ elsewhere. This is called the stabilization of $\tau$. Then $\widehat{\mathcal{D D}}(\tau)$ and $\widehat{\mathcal{D D}}(\stackrel{\circ}{\tau})$ are related as follows: fix any idempotent $i_{o}$ on $\mathcal{Z}^{1}$ (occupying one of the two possible pairs), then there is an injection from generators of $\widehat{\mathcal{D D}}(\tau)$ into generators of $\widehat{\mathcal{D D}}\left(\frac{\circ}{\tau}\right)$, sending $i \otimes i^{\prime}$ in $\widehat{\mathcal{D D}}(\tau)$ to $\left(i \# i_{o}\right) \otimes\left(i^{\prime} \# \overline{o\left(i_{o}\right)}\right)$ in $\widehat{\mathcal{D D}}\left(\frac{\circ}{\tau}\right)$. For any generator $\boldsymbol{x}$ in $\widehat{\mathcal{D D}}(\tau)$, let $\stackrel{\circ}{\boldsymbol{x}}$ be the corresponding generator in $\widehat{\mathcal{D D}}(\stackrel{\circ}{\tau})$. Then for any two generators $\boldsymbol{x}, \boldsymbol{y}$ in $\widehat{\mathcal{D D}}(\tau)$, there is a one-to-one correspondence between arrows from $\boldsymbol{x}$ to $\boldsymbol{y}$ in the $D D$ action of $\widehat{\mathcal{D D}}(\tau)$ and arrows from $\stackrel{\circ}{\boldsymbol{x}}$ to $\stackrel{\circ}{\mathrm{y}}$ in the $D D$ action of $\widehat{\mathcal{D D}}(\stackrel{\circ}{\tau})$ that do not cover any region around the adjoined $\mathcal{Z}^{1}$, with $\boldsymbol{x} \rightarrow a \otimes b \otimes \boldsymbol{y}$ corresponding to $\stackrel{\circ}{\boldsymbol{x}} \rightarrow \stackrel{\circ}{a} \otimes \stackrel{\circ}{b} \otimes \stackrel{\circ}{\boldsymbol{y}}$, where $\stackrel{\circ}{a}$ and $\stackrel{\circ}{b}$ are obtained from $a$ and $b$ by adjoining the appropriate idempotents.

Duality For any arcslide $\tau: F^{\circ}\left(\mathcal{Z}_{1}\right) \rightarrow F^{\circ}\left(\mathcal{Z}_{2}\right)$, let $\bar{\tau}: F^{\circ}\left(-\mathcal{Z}_{1}\right) \rightarrow F^{\circ}\left(-\mathcal{Z}_{2}\right)$ be the arcslide with reversed orientation. Now we have that $\mathcal{A}\left(\mathcal{Z}_{1}\right), \mathcal{A}\left(-\mathcal{Z}_{2}\right) \widehat{\mathcal{D D}}(\tau)$ and $\mathcal{A}\left(-\mathcal{Z}_{1}\right), \mathcal{A}\left(\mathcal{Z}_{2}\right) \widehat{\mathcal{D D}}(\bar{\tau})$ are dual to each other (using definition of dual at the end of Section $2 \mathrm{~A})$; that is,

$$
\overline{\mathcal{A}\left(\mathcal{Z}_{1}\right), \mathcal{A}\left(-\mathcal{Z}_{2}\right) \widehat{\mathcal{D D}}(\tau)} \simeq \mathcal{A}\left(-\mathcal{Z}_{1}\right), \mathcal{A}\left(\mathcal{Z}_{2}\right) \widehat{\mathcal{D D}}(\bar{\tau})
$$

Furthermore, the invariant $\mathcal{A}\left(\mathcal{Z}_{2}\right), \mathcal{A}\left(-\mathcal{Z}_{1}\right) \widehat{\mathcal{D D}}\left(\tau^{-1}\right)$ is homotopy equivalent to the right side of (15), after switching the two algebra actions. This comes from the fact that the mapping cylinder of $\tau^{-1}$ is the mirror image of the mapping cylinder of $\tau$. 


\section{F Main constructions}

We now summarize the combinatorial constructions that will be studied in this paper. From here on, we will no longer use the analytical definitions of invariants, but define everything combinatorially from scratch. We will use notations such as $\widehat{\mathrm{CFA}}$ A to denote (combinatorially defined) homotopy equivalence classes of bimodules, and notations such as $\widehat{\mathcal{D D}}, \widehat{\mathcal{D A}}$ to denote particular combinatorial models in the equivalence classes of bimodules. For modules with one algebra action, we will use $\widehat{\mathrm{CFA}}$ and $\widehat{\mathrm{CFD}}$ for both models and equivalence classes, as no confusion will arise there. Since all combinatorial definitions below use either constructions derived from the analytical definition, or the appropriate box tensor product, it is clear that the entire construction agrees with the analytical definitions.

First, we will use models $\widehat{\mathcal{D D}}\left(\mathbb{I}_{\mathcal{Z}}\right), \mathcal{A ( \mathcal { Z } )} \mathbb{I}_{\mathcal{A}(\mathcal{Z})}$, and $\widehat{\mathcal{A A}}\left(\mathbb{I}_{\mathcal{Z}}\right)$ to define $\widehat{\operatorname{CFDD}}\left(\mathbb{I}_{\mathcal{Z}}\right)$, $\widehat{\mathrm{CFDA}}\left(\mathbb{I}_{\mathcal{Z}}\right)$, and $\widehat{\mathrm{CFA} A}\left(\mathbb{I}_{\mathcal{Z}}\right)$, respectively. Then Corollary 3.10 shows (12) holds for our combinatorial construction. This means box tensoring with $\widehat{C F D D}\left(\mathbb{I}_{\mathcal{Z}}\right)$ and $\widehat{\mathrm{CFA} A}\left(\mathbb{I}_{\mathcal{Z}}\right)$ are inverse operations on equivalence classes of bimodules.

Next, we define $\widehat{\mathcal{D A}}(\tau)$ as the box tensor product

$$
\mathcal{A}\left(\mathcal{Z}_{1}\right) \widehat{\mathcal{D A}}(\tau)_{\mathcal{A}\left(\mathcal{Z}_{2}\right)}=\widehat{\mathcal{A A}}\left(\mathbb{I}_{\mathcal{Z}_{2}}\right)_{\mathcal{A}\left(-\mathcal{Z}_{2}\right), \mathcal{A}\left(\mathcal{Z}_{2}\right)} \otimes_{\mathcal{A}\left(-\mathcal{Z}_{2}\right)} \mathcal{A}\left(\mathcal{Z}_{1}\right), \mathcal{A}\left(-\mathcal{Z}_{2}\right) \widehat{\mathcal{D D}}(\tau)
$$

Given this, we can define $\widehat{\mathcal{D A}}(\phi)$ for an arbitrary element $\phi$ of the strongly based mapping class groupoid, by factoring $\phi$ into arcslides. The precise statement is the following.

Construction 2.1 Given an element $\phi: F^{\circ}\left(\mathcal{Z}_{1}\right) \rightarrow F^{\circ}\left(\mathcal{Z}_{n+1}\right)$ of the strongly based mapping class groupoid, with factorization $\phi=\tau_{n} \circ \cdots \circ \tau_{1}$, where $\tau_{i}: F^{\circ}\left(\mathcal{Z}_{i}\right) \rightarrow$ $F^{\circ}\left(\mathcal{Z}_{i+1}\right)$. Write $\tau$ for the sequence $\tau_{1}, \ldots, \tau_{n}$. Define

$$
\mathcal{A}\left(\mathcal{Z}_{1}\right) \widehat{\mathcal{D A}}(\phi, \tau)_{\mathcal{A}\left(\mathcal{Z}_{n+1}\right)}={ }^{\mathcal{A}\left(\mathcal{Z}_{1}\right)} \widehat{\mathcal{D A}}\left(\tau_{1}\right)_{\mathcal{A}\left(\mathcal{Z}_{2}\right)} \nabla \cdots \nabla^{\mathcal{A}\left(\mathcal{Z}_{n}\right)} \widehat{\mathcal{D A}}\left(\tau_{n}\right)_{\mathcal{A}\left(\mathcal{Z}_{n+1}\right)} .
$$

Theorem 2.2 The homotopy type of $\widehat{\mathcal{D A}}(\phi, \tau)$ does not depend on the choice of factorization $\boldsymbol{\tau}$. Hence, $\widehat{\mathcal{D A}}(\phi, \tau)$ is an invariant of $\phi$ up to homotopy equivalence.

This theorem is proven in Section 4. Given this, we can define $\widehat{\operatorname{CDDA}}(\phi)$ to be the equivalence class of $\widehat{\mathcal{D A}}(\phi, \tau)$, for any choice of factorization $\boldsymbol{\tau}$.

The other bimodule invariants $\widehat{\operatorname{CFDD}}(\phi), \widehat{\mathrm{CFA} A}(\phi)$, and $\widehat{\mathrm{CFAD}}(\phi)$ for a general morphism $\phi: F^{\circ}\left(\mathcal{Z}_{1}\right) \rightarrow F^{\circ}\left(\mathcal{Z}_{2}\right)$ can be defined as follows:

$$
\begin{aligned}
& \mathcal{A}\left(\mathcal{Z}_{1}\right), \mathcal{A}\left(-\mathcal{Z}_{2}\right) \widehat{\mathrm{CFDD}}(\phi)={ }^{\mathcal{A}\left(\mathcal{Z}_{1}\right)} \widehat{\mathrm{CFDA}}(\phi)_{\mathcal{A}\left(\mathcal{Z}_{2}\right)} \nabla_{\mathcal{A}\left(\mathcal{Z}_{2}\right)} \mathcal{A ( \mathcal { Z } _ { 2 } ) , \mathcal { A } ( - \mathcal { Z } _ { 2 } )} \widehat{\mathrm{CFDD}}\left(\mathbb{I}_{\mathcal{Z}_{2}}\right) \text {, } \\
& \widehat{\mathrm{CFA} A}(\phi)_{\mathcal{A}\left(-\mathcal{Z}_{1}\right), \mathcal{A}\left(\mathcal{Z}_{2}\right)}=\widehat{\mathrm{CFA}}\left(\mathbb{I}_{\mathcal{Z}_{1}}\right)_{\mathcal{A}\left(-\mathcal{Z}_{1}\right), \mathcal{A}\left(\mathcal{Z}_{1}\right)} \otimes_{\mathcal{A}\left(\mathcal{Z}_{1}\right)} \mathcal{A ( \mathcal { Z } _ { 1 } )} \widehat{\operatorname{CFDA}}(\phi)_{\mathcal{A}\left(\mathcal{Z}_{2}\right)}, \\
& \mathcal{A}\left(-\mathcal{Z}_{2}\right) \widehat{\mathrm{CFAD}}(\phi)_{\mathcal{A}\left(-\mathcal{Z}_{1}\right)}=\widehat{\mathrm{CFA}}\left(\mathbb{I}_{\mathcal{Z}_{1}}\right)_{\mathcal{A}\left(-\mathcal{Z}_{1}\right), \mathcal{A}\left(\mathcal{Z}_{1}\right)} \otimes_{\mathcal{A}\left(\mathcal{Z}_{1}\right)} \mathcal{A}\left(\mathcal{Z}_{1}\right), \mathcal{A}\left(-\mathcal{Z}_{2}\right) \widehat{\mathrm{CFDD}}(\phi) \text {. }
\end{aligned}
$$




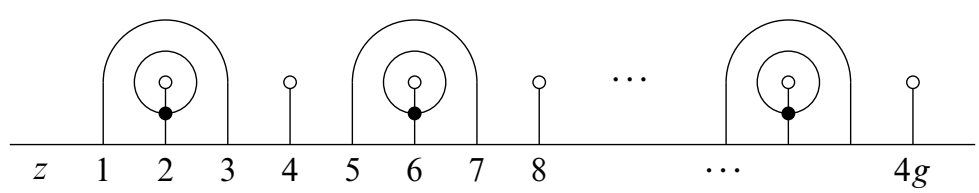

Figure 4: Heegaard diagram for the 0-framed handlebody. The numbers at bottom label points in $\mathcal{Z}^{g}$. Points in $-\mathcal{Z}^{g}$ are labeled in the reverse order.

Since $\widehat{\mathcal{D D}}\left(\mathbb{I}_{\mathcal{Z}}\right)$ is the quasi-inverse of $\widehat{\mathcal{A A}}\left(\mathbb{I}_{\mathcal{Z}}\right)$ (that is, inverse up to homotopy equivalence), we know $\widehat{\mathrm{CFDD}}(\tau)$ for an arcslide $\tau$ can also be represented by $\widehat{\mathcal{D D}}(\tau)$. Also, expanding out the definitions, we see $\widehat{\operatorname{CFAD}}(\phi) \simeq \widehat{\mathrm{CFA} A}(\phi) \otimes \widehat{\operatorname{CFDD}}\left(\mathbb{I}_{\mathcal{Z}_{2}}\right)$.

This concludes our construction of bimodule invariants (we will not need bimodule invariants other than those for mapping classes of surface diffeomorphisms). To construct invariants of closed 3-manifolds, we need one more building block: $\widehat{\mathrm{CFD}}$ of the 0 -framed handlebody $\boldsymbol{H}^{g}$. Here $\boldsymbol{H}^{g}$ is the 3 -manifold with one parametrized boundary given by the Heegaard diagram in Figure 4.

In this diagram, the small circles are 1-handle attachment points, paired consecutively. The larger circles are $\beta$ circles, and all other arcs inside the boundary are $\alpha$ arcs. From the way the $\alpha$ arcs meet the boundary, we see that the boundary of $\boldsymbol{H}^{g}$ is parametrized by the split pointed matched circle of genus $g$, denoted $\mathcal{Z}^{g}$. This is the pointed matched circle with matching

$$
(1,3),(2,4),(5,7),(6,8), \ldots,(4 g-3,4 g-1),(4 g-2,4 g) .
$$

While it is true that $-\mathcal{Z}^{g}=\mathcal{Z}^{g}$, we will usually distinguish them in order to emphasize orientation changes.

The orientation reversal $-\boldsymbol{H}^{g}$ is called the $\infty$-framed handlebody. Its boundary is parametrized by $-\mathcal{Z}^{g}$. The Heegaard diagram for $-\boldsymbol{H}^{g}$ is the reflection of that for $\boldsymbol{H}^{g}$.

The invariant $\widehat{\mathrm{CFD}}\left(\boldsymbol{H}^{g}\right)$ has left type $D$ action by $\mathcal{A}\left(-\mathcal{Z}^{g}\right)$. It can be defined using the following model: there is a single generator $\boldsymbol{x}$, corresponding to the set of intersection points indicated in Figure 4. The idempotent of $\boldsymbol{x}$ contains pairs $(2,4),(6,8), \ldots$ in $-\mathcal{Z}^{g}$ (pairs corresponding to $\alpha$-arcs not occupied by $\boldsymbol{x}$; note the labeling of points in $-\mathcal{Z}^{g}$ is reversed). The type $D$ action is

$$
\delta^{1}(\boldsymbol{x})=\sum_{\xi \in \mathcal{D}} a(\xi) \cdot \boldsymbol{x}
$$

where $\mathcal{D}$ is the set of chords $\{2 \rightarrow 4,6 \rightarrow 8, \ldots\}$. The invariant $\widehat{\mathrm{CFD}}\left(-\boldsymbol{H}^{g}\right)$ can be defined to be the dual of $\widehat{\mathrm{CFD}}\left(\boldsymbol{H}^{g}\right)$. 
We now give the combinatorial construction of $\widehat{\mathrm{HF}}(Y)$ for a closed 3-manifold $Y$, following the spirit of the construction in [11].

Construction 2.3 Let $Y$ be a closed 3-manifold. Choose a Heegaard splitting $Y_{1} \cup_{u} Y_{2}$ of $Y$, where $u: \partial Y_{1} \rightarrow-\partial Y_{2}$ is the gluing map. Fix circle and basepoint $(Z, z)$ on the gluing boundary $Y_{1} \cap Y_{2}$, and diffeomorphisms $f_{1}: \boldsymbol{H}^{g} \rightarrow Y_{1}$ and $f_{2}:-\boldsymbol{H}^{g} \rightarrow Y_{2}$, preserving $(Z, z)$, from the standard handlebodies to $Y_{1}$ and $Y_{2}$. Let $f_{1 *}: F^{\circ}\left(\mathcal{Z}^{g}\right) \rightarrow$ $\partial Y_{1}$ and $f_{2 *}: F^{\circ}\left(-\mathcal{Z}^{g}\right) \rightarrow \partial Y_{2}$ be the restrictions of $f_{1}$ and $f_{2}$ to the boundary. Let $\psi=\bar{f}_{2 *}^{-1} \circ u \circ f_{1 *}$ be the induced gluing map. This is an element of the strongly based mapping class group on $F^{\circ}\left(\mathcal{Z}^{g}\right)$. Define

$\widehat{\mathrm{HF}}\left(Y, Y_{1}, Y_{2}, u, f_{1}, f_{2}\right)$

$$
=\left(\widehat{\mathrm{CFA}} \widehat{A}(\psi)_{\mathcal{A}\left(-\mathcal{Z}^{g}\right), \mathcal{A}\left(\mathcal{Z}^{g}\right)} \otimes^{\mathcal{A}\left(-\mathcal{Z}^{g}\right)} \widehat{\mathrm{CFD}}\left(\boldsymbol{H}^{g}\right)\right) \otimes^{\mathcal{A}\left(\mathcal{Z}^{g}\right)} \widehat{\mathrm{CFD}}\left(-\boldsymbol{H}^{g}\right) .
$$

Theorem 2.4 The homotopy type of $\widehat{\mathrm{HF}}\left(Y, Y_{1}, Y_{2}, u, f_{1}, f_{2}\right)$ does not depend on the choice of Heegaard splitting $Y=Y_{1} \cup_{u} Y_{2}$ or the parametrizations $f_{1}, f_{2}$. Therefore it gives an invariant of $Y$ up to homotopy equivalence.

We will prove Theorem 2.4 combinatorially in Section 5. Given this theorem, we can write $\widehat{\mathrm{HF}}(Y)$ for $\widehat{\mathrm{HF}}\left(Y, Y_{1}, Y_{2}, u, f_{1}, f_{2}\right)$, for some choice of Heegaard splitting and parametrizations. From the construction, it is clear that this is equivalent to the definition of $\widehat{\mathrm{HF}}(Y)$ using holomorphic curves.

\section{Computations on $D A$ invariants}

In this section, we prove some preliminary results on type $D A$ bimodules, and perform some computations on the type $D A$ invariants of arcslides, in preparation for the proof of Theorem 2.2 in Section 4.

First, we give an outline for the proof of Theorem 2.2. We want to show that the combinatorial construction of $\widehat{\mathcal{D A}}(\phi, \tau)$ does not depend on the choice of factorization of $\phi$ into arcslides $\tau$. For this purpose, it is necessary to understand relations among arcslides. This is studied in detail in $[1 ; 2]$. The notions of pointed matched circles and arcslides correspond to linear chord diagrams and chord slides in these papers. We now give a summary of the results.

Locally, an arcslide can be viewed as one end of the $B$ pair sliding along the $C$ pair:

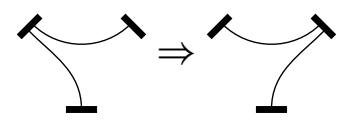


In this diagram, the three short segments denote portions of the straight line in the pointed matched circle. The upper, stationary arc denotes the $C$ pair; and the lower, moving arc denotes the $B$ pair.

There are five types of relations on arcslides, and together they generate all relations. The local diagrams for the five types of relations are as follows (see [2, Theorem 6.2, Figure 6.1]):

- Triangle

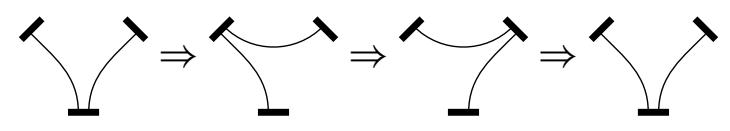

- Involution

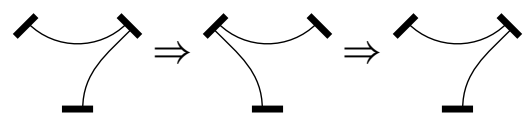

- Commutativity

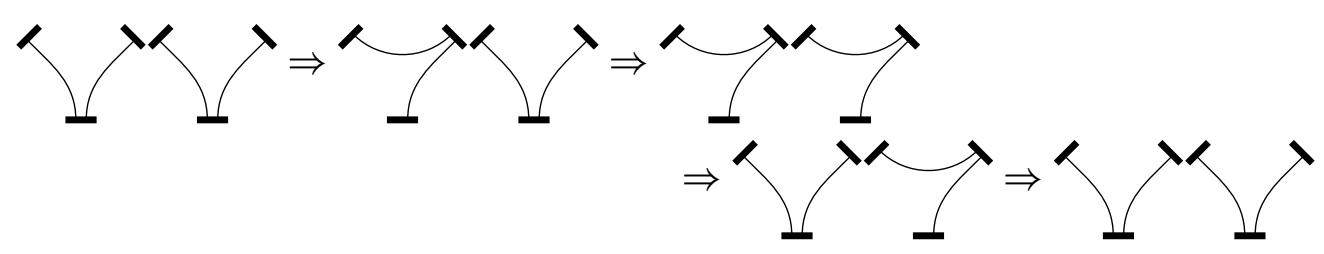

- Left pentagon

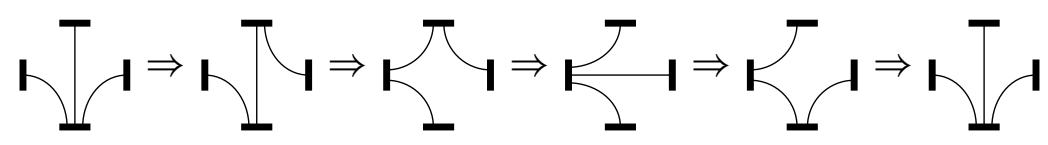

- Right pentagon

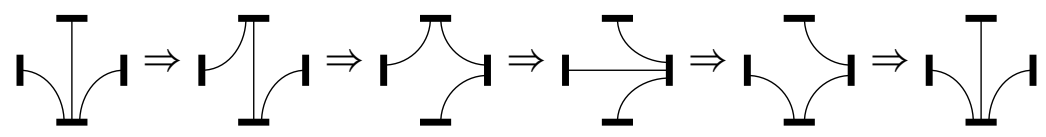

Each relation gives one way to factor the identity morphism $\mathbb{I}_{\mathcal{Z}}$ starting and ending at some pointed matched circle $\mathcal{Z}$. For proving Theorem 2.2, it suffices to check that for each such factorization

$$
\mathbb{I}_{\mathcal{Z}}=\tau_{n} \circ \cdots \circ \tau_{1},
$$

the corresponding homotopy equivalence

$$
\mathcal{A}(\mathcal{Z}) \mathbb{I}_{\mathcal{A}(\mathcal{Z})} \simeq \widehat{\mathcal{D A}}\left(\tau_{1}\right) \otimes \cdots \otimes \widehat{\mathcal{D A}}\left(\tau_{n}\right)
$$

holds. Note that in general, the starting and ending pointed matched circles of each $\tau_{i}$ may be different from $\mathcal{Z}$. This is the main reason why we need to consider strongly 
based mapping class groupoids, even if we are only interested in statements about strongly based mapping class groups.

The overall strategy for verifying (17) is as follows: from the description of $\widehat{\mathcal{D A}}\left(\tau_{i}\right)$, we can readily enumerate the set of generators on the right side of the equation. There are, however, more generators on the right side than on the left side. The cancellation lemma for type $D A$ bimodules describes conditions under which we can prove that a bimodule is homotopy equivalent to one with two fewer generators. Using it, we can remove generators from the right side in pairs, so that the set of remaining generators matches that on the left side. The cancellation lemma is stated and proven in Section 3A. It turns out that a type $D A$ bimodule with the same set of generators as $\mathcal{A ( Z )} \mathbb{I}_{\mathcal{A}(\mathcal{Z})}$, and with a few more properties in common with $\mathcal{A}(\mathcal{Z}) \mathbb{I}_{\mathcal{A}(\mathcal{Z})}$, must be homotopy equivalent to $\mathcal{A}(\mathcal{Z}) \mathbb{I}_{\mathcal{A}(\mathcal{Z})}$. We prove two lemmas of this kind, which we call rigidity lemmas, in Section 3B. The first lemma will be used to prove the involution relation, and the second lemma will be used for all other relations. The idea here is that once the involution relation is proven, we can show that $\widehat{\mathcal{D A}}(\tau)$ is quasi-invertible for any arcslide $\tau$, which implies that any box tensor product of such bimodules is also quasi-invertible (recall that a type $D A$ bimodule ${ }^{A} M_{B}$ is quasi-invertible if there exists ${ }^{B} N_{A}$ such that ${ }^{A} M_{B} \otimes^{B} N_{A} \simeq{ }^{A} \mathbb{I}_{A}$ ). This means checking the quasi-invertibility condition in the second lemma becomes trivial, and we can avoid checking the more involved condition in the first lemma that it replaces. We note here that the rigidity lemmas depend on specific properties of $\mathcal{A}(\mathcal{Z})$, and is not applicable to DG algebras in general.

To apply the cancellation lemma, and in the case of the involution relation, the rigidity lemma, we need to compute certain arrows in the type $D A$ action of the bimodule on the right side. To prepare for this, we review the construction of $\widehat{\mathcal{A A}}\left(\mathbb{I}_{\mathcal{Z}}\right)$ in Section $3 \mathrm{C}$, and compute in Section 3D some arrows in the type $D A$ action of $\widehat{\mathcal{D A}}(\tau)$ for arcslides $\tau$ (the components in the tensor product).

\section{A Cancellation lemmas}

In this section we state cancellation lemmas for type $D$ modules and type $D A$ bimodules over DG algebras. Both are generalizations of the cancellation lemma in the case of chain complexes. These results are well known; see, for example, [8, Section 2.6].

Let $A$ be a DG algebra over a ground ring $\boldsymbol{k}$, where $\boldsymbol{k}$ is a direct sum of copies of $\mathbb{F}_{2}$ (in our application, $A=\mathcal{A}(\mathcal{Z})$ and $\boldsymbol{k}$ is generated by the indecomposable idempotents). Let $M$ be a left type $D$ module over $A$ with a fixed set of generators $\mathcal{G}$. We can describe the action $\delta^{1}$ on $M$ in terms of coefficients as follows: for any $x \in \mathcal{G}$, expand $\delta^{1}(x)$ as

$$
\delta^{1}(x)=\sum_{\boldsymbol{y} \in \mathcal{G}} c_{x y} \otimes \boldsymbol{y}
$$




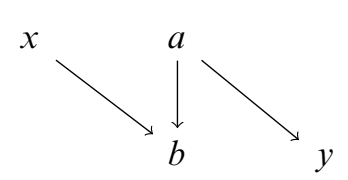

Figure 5: Standard example of a zigzag in $M$. This becomes $x \rightarrow$ $c_{x b} c_{a b}^{-1} c_{a y} \otimes y$ in $M^{\prime}$.

for some choice of $c_{x y}$.

Here the tensor product is implicitly taken over $\boldsymbol{k}$, and as a result there is some flexibility in the choice of $c_{x y}$. We generally choose $c_{x y}$ to consist of as few generators of $A$ as possible, except when choosing $c_{x y}$ to be invertible whenever possible.

Now suppose that for some $a, b \in \mathcal{G}$, the coefficient $c_{a b}$ is invertible in $A$, and $d\left(c_{a b}\right)=0$. Then there is a new type $D$ module $M^{\prime}$, generated by $\mathcal{G}^{\prime}=\mathcal{G} \backslash\{a, b\}$ and with type $D$ action

$$
\delta^{1^{\prime}}(x)=\sum_{y \in \mathcal{G}^{\prime}}\left(c_{x y}+c_{x b} c_{a b}^{-1} c_{a y}\right) \otimes y
$$

for any $x \in \mathcal{G}^{\prime}$. The first part of each term in the sum is simply the original $\delta^{1}$ (excluding terms involving $a$ and $b$ ). The second part is as follows: for each zigzag in $M$, as shown in Figure 5, the term $c_{x b} c_{a b}^{-1} c_{a y} \otimes y$ is added to $\delta^{1^{\prime}} x$. The coefficient can be read out by following the arrows from $x$ to $y$, treating a reversed arrow as taking inverse.

Theorem 3.1 (cancellation lemma for type $D$ modules) With the above definitions, the action $\delta^{1^{\prime}}$ on $M^{\prime}$ satisfies the type $D$ structure equation, and the resulting type $D$ module $M^{\prime}$ is homotopy equivalent to $M$.

Proof We prove this by giving explicit type $D$ morphisms and homotopies, in terms of coefficients as we did for the type $D$ action. The necessary data are morphisms $f: M \rightarrow M^{\prime}$ and $g: M^{\prime} \rightarrow M$, and homotopy $h: M \rightarrow M$, satisfying the identities $f \circ g=\mathbb{I}_{M^{\prime}}$ and $g \circ f=\mathbb{I}_{M}+h \circ \delta^{1}+\delta^{1} \circ h$. The morphisms $f$ and $g$ are given by

$$
\begin{gathered}
f(a)=0, \quad f(b)=\sum_{y \in \mathcal{G}^{\prime}} c_{a b}^{-1} c_{a y} \otimes y, \quad \text { and } \quad f(x)=1 \otimes x \text { for } x \in \mathcal{G}^{\prime} ; \\
g(x)=1 \otimes x+c_{x b} c_{a b}^{-1} \otimes a .
\end{gathered}
$$

Part of $f$ can be visualized using the zigzag by following arrows from $b$ to $y$. Likewise, part of $g$ can be visualized by following arrows from $x$ to $a$. The homotopy $h: M \rightarrow M$ is given by $h(b)=c_{a b}^{-1} \otimes a$ and $h(x)=0$ for any $x \neq b$. 
It remains to verify that $\delta^{1^{\prime}}$ satisfies the type $D$ structure equations, and the maps $f, g$, and $h$ satisfy the required identities. This can be done by converting the equations into their coefficient form. For example, the type $D$ structure equation

$$
\left(\mu_{2} \otimes \mathbb{I}_{M}\right) \circ\left(\mathbb{I}_{A} \otimes \delta^{1}\right) \circ \delta^{1}+\left(\mu_{1} \otimes \mathbb{I}_{M}\right) \circ \delta^{1}=0,
$$

when written in terms of coefficients, becomes

$$
d\left(c_{x y}\right)+\sum_{z \in \mathcal{G}} c_{x z} c_{z y}=0
$$

for any $x, y \in \mathcal{G}$.

The structure equation for a type $D$ morphism $\phi: M \rightarrow N$ is

$$
\left(\mu_{2} \otimes \mathbb{I}_{N}\right) \circ\left(\mathbb{I}_{A} \otimes \delta_{N}^{1}\right) \circ \phi^{1}+\left(\mu_{2} \otimes \mathbb{I}_{N}\right) \circ\left(\mathbb{I}_{A} \otimes \phi^{1}\right) \circ \delta_{M}^{1}+\left(\mu_{1} \otimes \mathbb{I}_{N}\right) \circ \phi^{1}=0 .
$$

For any $x \in \mathcal{G}(M)$ and $y \in \mathcal{G}(N)$, let $\phi_{x y}$ be the coefficient of $y$ in $\phi^{1}(x)$. Then, applying the above equation to an arbitrary generator $x$ of $M$, we see that the structure equation is equivalent to

$$
d\left(\phi_{x y}\right)+\sum_{z^{\prime} \in \mathcal{G}(N)} \phi_{x z^{\prime}} c_{z^{\prime} y, N}+\sum_{z \in \mathcal{G}(M)} c_{x z, M} \phi_{z y}=0
$$

for any $y \in \mathcal{G}(N)$.

The composition of two morphisms $\phi: M \rightarrow N$ and $\psi: N \rightarrow P$ is given by

$$
(\psi \circ \phi)^{1}=\left(\mu_{2} \otimes \mathbb{I}_{P}\right) \circ\left(\mathbb{I}_{A} \otimes \psi^{1}\right) \circ \phi^{1} .
$$

In terms of coefficients, this is

$$
(\psi \circ \phi)_{x y}=\sum_{z \in \mathcal{G}(N)} \phi_{x z} \psi_{z y}
$$

for any $x \in \mathcal{G}(M)$ and $y \in \mathcal{G}(P)$.

It is then routine to verify these equations, using the assumption that $c_{a b}$ is invertible and $d\left(c_{a b}\right)=0$.

The cancellation lemma for type $D A$ bimodules follows from that for type $D$ modules, by viewing type $D A$ bimodules over $A^{\prime}$ and $A$ as type $D$ modules over $\widehat{\operatorname{Cob}}(A) \otimes A^{\prime}$; see [12, Remark 2.2.35].

Definition 3.2 Given a strand algebra $A$, let $A_{+}$be the DG subalgebra of $A$ generated by the nonidempotent generators. The cobar resolution $\operatorname{Cob}(A)$ is defined as $T^{*}\left(A_{+}[1]^{*}\right)$, the tensor algebra of the dual of $A_{+}$. This can be given the structure of 
a DG algebra, whose product is that of tensor algebra, and whose differential consists of the following arrows:

- $\quad a_{1}^{*} \otimes \cdots \otimes b^{*} \otimes \cdots \otimes a_{k}^{*} \rightarrow a_{1}^{*} \otimes \cdots \otimes a_{i}^{*} \otimes \cdots \otimes a_{k}^{*}$ for each $i$ and term $b$ in $d a_{i}$,

- $a_{1}^{*} \otimes \cdots \otimes a_{i}^{*} \otimes \cdots \otimes a_{k}^{*} \rightarrow a_{1}^{*} \otimes \cdots \otimes b^{*} \otimes b^{*} \otimes \cdots \otimes a_{k}^{*}$ for each $i$ and generators $b, b^{\prime}$ such that $b b^{\prime}=a_{i}$.

Furthermore, we write $\widehat{\operatorname{Cob}}(A)$ to denote the completion of $\operatorname{Cob}(A)$ with respect to the length filtration, that is, an element of $\widehat{\operatorname{Cob}}(A)$ is a formal sum of elements in $\left(A_{+}[1]^{*}\right)^{\otimes i}$ for possibly infinitely many $i$.

The category of type $D A$ bimodules over $A^{\prime}$ on the $D$-side and $A$ on the $A$-side is equivalent to the category of type $D$ modules over $\widehat{\operatorname{Cob}}(A) \otimes A^{\prime}$, where the arrow

$$
\delta_{1+i}^{1}:\left(x ; a_{1}, \ldots, a_{i}\right) \rightarrow a^{\prime} \otimes y
$$

in the action of a type $D A$ bimodule $M$ corresponds to the arrow

$$
\delta^{1}: x \rightarrow\left(a_{1}^{*} \otimes \cdots \otimes a_{i}^{*}\right) \otimes a^{\prime} \otimes y
$$

in the action of the type $D$ module corresponding to $M$.

Using this correspondence, we can define coefficients on a type $D A$ bimodule.

Definition 3.3 Given two generators $x, y$ of $M$, define the coefficient $C_{x y}$ to be the formal sum, in $\widehat{\operatorname{Cob}}\left(A^{\prime}\right) \otimes A$, of $\left(a_{1}^{*} \otimes \cdots \otimes a_{i}^{*}\right) \otimes a^{\prime}$ over all arrows of the form $\delta_{1+i}^{1}:\left(x ; a_{1}, \ldots, a_{i}\right) \rightarrow a^{\prime} \otimes y$. As in the type $D$ case, we choose $a^{\prime}$ to be invertible whenever possible when writing the action in terms of arrows.

This allows us to state the cancellation lemma for type $D A$ bimodules, following immediately from the cancellation lemma in the type $D$ case, and the equivalence of categories.

Theorem 3.4 (cancellation lemma for type $D A$ bimodules) Let $A^{\prime} M_{A}$ be a type $D A$ bimodule, with a fixed set $\mathcal{G}$ of generators. Suppose there are $x, y \in \mathcal{G}$ such that $C_{x y}=1 \otimes a$ with $a \in A$ invertible and $d a=0$. Then $C_{x y}^{-1}=1 \otimes a^{-1}$, and the type $D A$ bimodule $M^{\prime}$ generated by $\mathcal{G}^{\prime}=\mathcal{G} \backslash\{x, y\}$ and with coefficients $C_{a b}^{\prime}=$ $C_{a b}+C_{a y} C_{x y}^{-1} C_{x b}$ is homotopy equivalent to $M$. 
We end with a remark on grading. If $M$ is graded by a grading set $S_{M}$, and if every generator being cancelled is homogeneous in grading, then $M^{\prime}$ is also graded by $S_{M}$, with the grading of each generator in $M^{\prime}$ equal to the grading of the corresponding generator in $M$. The arrows that are added to $M^{\prime}$ satisfy the grading constraints, because they come from traversing a zigzag as in Figure 5, where each of the three arrows in the zigzag satisfy the grading constraints. The homogeneity condition of the cancelled generators will be automatically satisfied in our case.

\section{B Characterization of the identity bimodule}

In this section, we prove two lemmas describing conditions under which we can assert a type $D A$ bimodule ${ }^{\mathcal{A}(\mathcal{Z})} M_{\mathcal{A}(\mathcal{Z})}$ is homotopy equivalent to the identity bimodule $\mathcal{A}(\mathcal{Z}) \mathbb{I}_{\mathcal{A}(\mathcal{Z})}$. The main result we use is the characterization of $\widehat{\mathcal{D D}}\left(\mathbb{I}_{\mathcal{Z}}\right)$ given in [11]. We will start by reviewing that result here.

Definition 3.5 [11, Definition 3.1] The diagonal subalgebra of $\mathcal{A}(\mathcal{Z}) \otimes \mathcal{A}(-\mathcal{Z})$ is the algebra generated by $a \otimes b$, where $a$ and $b$ satisfy the following conditions: $\operatorname{mult}(a)=\operatorname{mult}(\bar{b})$, the left idempotents of $a$ and $b$ are complementary, and the right idempotents of $a$ and $b$ are complementary.

Proposition 3.6 [11, Proposition 3.8, proof of Theorem 1] Let $M$ be a left type $D D$ bimodule over $\mathcal{A}(\mathcal{Z})$ and $\mathcal{A}(-\mathcal{Z})$, where $\mathcal{Z}$ has genus greater than one. Suppose $M$ satisfies the following conditions, then $M$ is isomorphic to $\widehat{\mathcal{D D}}\left(\mathbb{I}_{\mathcal{Z}}\right)$ :

(1) The generators of $M$ are in one-to-one correspondence with the idempotents of $\mathcal{A}(\mathcal{Z})$, so that the generator corresponding to idempotent $i$ has (type $D$ ) idempotents $i$ and $\overline{o(i)}$.

(2) For any arrow $\boldsymbol{x} \rightarrow a \otimes b \otimes \boldsymbol{y}$ in the differential of $M$, the element $a \otimes b$ lies in the diagonal subalgebra.

(3) $\quad M$ is graded by a $\lambda$-free grading set $S$, with a left-right $G(\mathcal{Z})-G(\mathcal{Z})$ action.

(4) The differential in $M$ contains all arrows of the form

$$
\boldsymbol{x} \rightarrow a(\xi) \otimes \overline{a(\xi)} \otimes \boldsymbol{y},
$$

where $\xi$ is a length-1 chord.

In the case where $\mathcal{Z}$ has genus one, if $M$ satisfies an additional stability condition, in the sense of [11, Definition 1.8], then we can still conclude that $M=\widehat{\mathcal{D D}}\left(\mathbb{I}_{\mathcal{Z}}\right)$.

The following result will be used in the proof of the second lemma. 
Proposition 3.7 Suppose a type $D A$ bimodule ${ }^{\mathcal{A}(\mathcal{Z})} M_{\mathcal{A}(\mathcal{Z})}$ satisfies the following two conditions:

(1) $M$ is homotopy equivalent to the identity bimodule $\mathcal{A ( Z )} \mathbb{I}_{\mathcal{A}(\mathcal{Z})}$.

(2) The generators of $M$ are in one-to-one correspondence with the idempotents of $\mathcal{A}(\mathcal{Z})$, so that the generator corresponding to idempotent $i$ has both left (type $D$ ) and right (type $A$ ) idempotent equal to $i$.

Then the type $D A$ action on $M$ contains all arrows of the form

$$
\delta_{2}^{1}:(\boldsymbol{x}, a(\xi)) \rightarrow a(\xi) \otimes \boldsymbol{y},
$$

where $\xi$ is a length-1 chord.

Proof Consider generators $\boldsymbol{x}, \boldsymbol{y}$ corresponding to idempotents $i, j \in \mathcal{A}(\mathcal{Z})$, and $\xi$ a length- 1 chord, such that the idempotent matches in the arrow (24). We want to show that (24) does exist as an arrow.

Let $T_{D}$ be a type $D$ module over $\mathcal{A}(\mathcal{Z})$ with two generators $\boldsymbol{x}_{D}$ and $\boldsymbol{y}_{D}$, whose idempotents are $i$ and $j$, such that $\delta^{1}\left(\boldsymbol{x}_{D}\right)=a(\xi) \otimes \boldsymbol{y}_{D}$ and $\delta^{1}\left(\boldsymbol{y}_{D}\right)=0$. Since $d(a(\xi))=0$, it is clear that $\delta^{1}$ satisfies the type $D$ structure equation.

Likewise, let $T_{A}$ be the $A_{\infty}$-module over $\mathcal{A}(\mathcal{Z})$ with two generators $\boldsymbol{x}_{A}$ and $\boldsymbol{y}_{A}$ whose idempotents are $i$ and $j$, and $m_{1,1}:\left(\boldsymbol{x}_{A}, a(\xi)\right) \rightarrow \boldsymbol{y}_{A}$ is the only arrow in the $A_{\infty}$-action.

Consider the tensor product $T_{A} \otimes N \otimes T_{D}$, with $N=M$ or $N=\mathbb{I}$. This is a chain complex with two generators $\boldsymbol{x}_{A} \otimes \boldsymbol{x} \otimes \boldsymbol{x}_{D}$ and $\boldsymbol{y}_{A} \otimes \boldsymbol{y} \otimes \boldsymbol{y}_{D}$, and there is an arrow between these two if and only if the arrow (24) exists in $N$ for the given $\boldsymbol{x}, \boldsymbol{y}$ and $a(\xi)$. In particular, $T_{A} \otimes \mathbb{I} \otimes T_{D}$ has zero homology. By assumption, $M \simeq \mathbb{I}$, so $T_{A} \otimes M \otimes T_{D}$ must also have zero homology. This shows the arrow (24) exists in $M$.

Remark The argument in the above proof only works when $\xi$ has length 1 . If otherwise, we may have $d(a(\xi)) \neq 0$, and $\delta^{1}$ on $T_{D}$ no longer satisfies the type $D$ structure equation. Indeed, in the case where $\xi$ has length 2 , we may have:

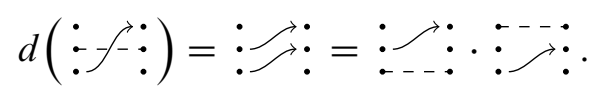

Hence, if there are generators $\boldsymbol{x}_{D}$ and $\boldsymbol{y}_{D}$ in $T_{D}$ with arrow $\boldsymbol{x}_{D} \rightarrow a(\xi) \otimes \boldsymbol{y}_{D}$, where the idempotents of $\boldsymbol{x}_{D}$ and $\boldsymbol{y}_{D}$ contain the middle point, then there must be an additional generator $z_{D}$ with appropriate arrows from $x_{D}$ to $z_{D}$ and from $z_{D}$ to $y_{D}$, so that the type $D$ structure equation remains satisfied. This is why we may have, for example, arrow (DA4) instead of (DA2) in ${ }^{\mathcal{A}(\mathcal{Z})} M_{\mathcal{A}(\mathcal{Z})}$, according the computations in Section 3D1. 
We now state and prove the lemmas on the characterization of ${ }^{\mathcal{A}(\mathcal{Z})} \mathbb{I}_{\mathcal{A}(\mathcal{Z})}$.

Lemma 3.8 Let $M={ }^{\mathcal{A}(\mathcal{Z})} M_{\mathcal{A}(\mathcal{Z})}$ be a left-right type $D A$ bimodule over $\mathcal{A}(\mathcal{Z})-\mathcal{A}(\mathcal{Z})$. Suppose $M$ satisfies the following properties, then $M$ is homotopy equivalent to the identity bimodule ${ }^{\mathcal{A}(\mathcal{Z})} \mathbb{I}_{\mathcal{A}(\mathcal{Z})}$.

- (ID-1) The generators of $M$ are in one-to-one correspondence with the idempotents of $\mathcal{A}(\mathcal{Z})$, so that the generator corresponding to idempotent $i$ has both left (type $D$ ) and right (type $A$ ) idempotent equal to $i$.

- (ID-2) $M$ can be graded by a principal left-right $G(\mathcal{Z})-G(\mathcal{Z})$ set, such that the induced map $\phi \in \operatorname{Out}(G(\mathcal{Z}), G(\mathcal{Z})$ ) (as in [11, Lemma 6.4]) is the identity map, and there is a choice of refined relative grading with every generator having grading zero. (The choice of grading refinement for $G(\mathcal{Z})$ is arbitrary but must be the same on both sides).

- (ID-3) The type DA action on $M$ contains all arrows of the form

$$
\delta_{2}^{1}:(\boldsymbol{x}, a(\xi)) \rightarrow a(\xi) \otimes \boldsymbol{y}
$$

where $\xi$ is a length-1 chord.

- (ID-4) $M$ is stable in the sense of [11, Definition 1.8] (this condition is only necessary when $\mathcal{Z}$ is the unique genus 1 pointed matched circle).

Proof Consider the type $D D$ bimodule $M_{D D}=M \otimes \widehat{\mathcal{D D}}\left(\mathbb{I}_{\mathcal{Z}}\right)$. We check that $M_{D D}$ satisfies all the conditions of Proposition 3.6, which will show that $M_{D D}$ is isomorphic to $\widehat{\mathcal{D D}}\left(\mathbb{I}_{\mathcal{Z}}\right)$. Since $\widehat{\mathcal{D D}}\left(\mathbb{I}_{\mathcal{Z}}\right)$ is quasi-invertible, this implies $M \simeq \mathbb{I}$.

Using the fact that relative grading can be chosen on $\widehat{\mathcal{D D}}\left(\mathbb{I}_{\mathcal{Z}}\right)$ so that every generator has grading zero, condition (ID-2) on the grading of $M$ implies a similar condition on the grading of $M_{D D}$. The constraint that the type $D D$ action must respect the grading implies that for each arrow

$$
\boldsymbol{x} \rightarrow a \otimes b \otimes \boldsymbol{y}
$$

the multiplicities of $a$ and $\bar{b}$ must be the same. The idempotent conditions on the diagonal subalgebra follow from the constraints on idempotents on each arrow, and the fact that both $\boldsymbol{x}$ and $\boldsymbol{y}$ have complementary idempotents. This verifies condition (2) of Proposition 3.6.

The other deductions are trivial. (ID-1), (ID-2) and (ID-3) imply conditions (1), (3) and (4), respectively. Condition (ID-4) implies the stability of $M_{D D}$, needed for the genus 1 case.

The condition (ID-3) in the previous lemma can still be difficult to verify in actual computations. It is possible to replace it as follows. 
Lemma 3.9 With the same notation as in Lemma 3.8, if $M$ satisfies the conditions (ID-1), (ID-2), (ID-4), and the following condition, then it is homotopy equivalent to $\mathbb{I}$.

- (ID-3') $M$ is invertible, with a quasi-inverse $M^{\prime}$ that satisfies y conditions (ID-1) and (ID-2).

Proof It suffices to show that (ID-3'), together with the other conditions, implies (ID-3). We first show that $\delta_{1}^{1}=0$ on both $M$ and $M^{\prime}$, that is, there are no arrows of the form

$$
\delta_{1}^{1}: x \rightarrow a \otimes y
$$

By the grading constraints on any arrow, the algebra generator $a$ must have multiplicity zero. That is, it must be an idempotent in $\mathcal{A}(\mathcal{Z})$. However, this would mean that the grading of $\boldsymbol{x}$ and $\boldsymbol{y}$ differ by $\lambda$, contradicting the assumption that all generators in $M$ (or $M^{\prime}$ ) have grading zero.

Both $M$ and its quasi-inverse $M^{\prime}$ also satisfy (ID-1), so by [12, Lemma 2.2.50] they can be represented as ${ }^{\mathcal{A}(\mathcal{Z})}[\phi]_{\mathcal{A}(\mathcal{Z})}$ and ${ }^{\mathcal{A}(\mathcal{Z})}\left[\phi^{\prime}\right]_{\mathcal{A}(\mathcal{Z})}$ respectively, for $A_{\infty}$-algebra morphisms $\phi, \phi^{\prime}: \mathcal{A}(\mathcal{Z}) \rightarrow \mathcal{A}(\mathcal{Z})$. Then $M^{\prime} \otimes M$ is represented by ${ }^{\mathcal{A}(\mathcal{Z})}\left[\phi^{\prime} \circ \phi\right]_{\mathcal{A}(\mathcal{Z})}$.

Since $M^{\prime} \otimes M$ satisfies the grading condition (ID-2), the map $\phi^{\prime} \circ \phi$ must preserve gradings. This means that for $a=a(\xi)$ where $\xi$ is a length- 1 chord, the only possible term in $\left(\phi^{\prime} \circ \phi\right)(a)$ is $a$. Since $M \otimes M^{\prime}$ is homotopy equivalent to identity, by Proposition 3.7, we have $\left(\phi^{\prime} \circ \phi\right)(a)=a$, which implies $\phi(a) \neq 0$. By the same grading argument, either $\phi(a)=0$ or $\phi(a)=a$. So we must have $\phi(a)=a$. This shows $\phi$ is the identity map on length- 1 chords, which implies condition (ID-3).

\section{C Combinatorial model of $\widehat{\mathrm{CFAA}}\left(\mathbb{I}_{\mathcal{Z}}\right)$}

In this section, we review the construction of the combinatorial model $\widehat{\mathcal{A A}}\left(\mathbb{I}_{\mathcal{Z}}\right)$ of $\widehat{\mathrm{CFA} A}\left(\mathbb{I}_{\mathcal{Z}}\right)$ given in [21], in preparation for computing some arrows in $\widehat{\mathcal{D A}}(\tau)$ for arcslides $\tau$ in the next section.

The construction begins with (11). After expanding the definitions, this gives a model of $\widehat{\mathrm{CFA} A}\left(\mathbb{I}_{\mathcal{Z}}\right)$ generated by the set of pairs $\left[a_{1}, a_{2}\right]$, where $a_{1}$ and $a_{2}$ are generators of $\mathcal{A}(\mathcal{Z})$, such that the initial idempotents of $a_{1}$ and $a_{2}$ are complementary. The differential and type $A A$ action on these generators are given as [21, Proposition 1]. The smaller model $\widehat{\mathcal{A A}}\left(\mathbb{I}_{\mathcal{Z}}\right)$ is obtained from this using homological perturbation theory. This involves finding the homology $C^{\prime}$ of $C$, the chain complex underlying the larger model, and giving chain maps $f: C \rightarrow C^{\prime}, g: C^{\prime} \rightarrow C$, and homotopy $H: C \rightarrow C$ verifying the homotopy equivalence between $C$ and $C^{\prime}$. The homology $C^{\prime}$ is generated by those $\left[a_{1}, a_{2}\right]$ where both $a_{1}$ and $a_{2}$ are idempotents (which are then 
complementary). The chain maps $f$ and $g$ are the obvious projection and inclusion maps. The homotopy $H$ is summarized in [21, Figures 6 and 9].

From homological perturbation theory, we obtain the following description of the smaller model: the $A_{\infty}$-bimodule $\widehat{\mathcal{A A}}\left(\mathbb{I}_{\mathcal{Z}}\right)_{\mathcal{A}(-\mathcal{Z}), \mathcal{A}(\mathcal{Z})}$ is generated by pairs of complementary idempotents $i^{\prime} \otimes i$, where $i \in \mathcal{A}(\mathcal{Z})$ and $i^{\prime}=\overline{o(i)} \in \mathcal{A}(-\mathcal{Z})$. The generator $i^{\prime} \otimes i$ has type $A$ idempotents $i^{\prime}$ and $i$. Each arrow in the type $A A$ action of $\widehat{\mathcal{A A}}\left(\mathbb{I}_{\mathcal{Z}}\right)$ comes from a sequence of moves between generators of $C$. There are three types of moves, the first two of which carry a coefficient.

- Move $A_{1}$ If $c \bar{b}^{\prime} \neq 0$, with $b^{\prime} \in \mathcal{A}(-\mathcal{Z})$, move from $\left[c \bar{b}^{\prime}, a_{2}\right]$ to $\left[c, a_{2}\right]$ with coefficient $b^{\prime}$.

- Move $\boldsymbol{A}_{2}$ If $a_{2} b \neq 0$, with $b \in \mathcal{A}(\mathcal{Z})$, move from $\left[a_{1}, a_{2}\right]$ to $\left[a_{1}, a_{2} b\right]$ with coefficient $b$.

- Move $\boldsymbol{H}$ Apply one of the arrows in the homotopy map $H$.

Each arrow then corresponds to a sequence $\left[a_{1,1}, a_{1,2}\right], \ldots,\left[a_{2 n, 1}, a_{2 n, 2}\right]$ of generators of $C$, satisfying the following conditions:

- $\left[a_{1,1}, a_{1,2}\right]=[o(i), i]$ and $\left[a_{2 n, 1}, a_{2 n, 2}\right]=[o(j), j]$ for some idempotents $i, j \in$ $\mathcal{A}(\mathcal{Z})$.

- Each $\left[a_{2 k, 1}, a_{2 k, 2}\right]$ is obtained from $\left[a_{2 k-1,1}, a_{2 k-1,2}\right]$ by applying either move $A_{1}$ or $A_{2}$.

- Each $\left[a_{2 k+1,1}, a_{2 k+1,2}\right]$ is obtained from $\left[a_{2 k, 1}, a_{2 k, 2}\right]$ by applying move $H$.

Let $b_{1}^{\prime}, \ldots, b_{p}^{\prime}$ be the ordered sequence of coefficients for moves of type $A_{1}$, and $b_{1}, \ldots, b_{q}$ be the ordered sequence of coefficients for moves of type $A_{2}$, then such a sequence of generators of $C$ gives rise to an arrow

$$
m_{1, p, q}:\left(i^{\prime} \otimes i ; b_{1}^{\prime}, \ldots, b_{p}^{\prime} ; b_{1}, \ldots, b_{q}\right) \rightarrow j^{\prime} \otimes j,
$$

where $i^{\prime}=\overline{o(i)}$ and $j^{\prime}=\overline{o(j)}$.

An important property of $\widehat{\mathcal{A A}}\left(\mathbb{I}_{\mathcal{Z}}\right)$, which follows directly from this construction, is that for any arrow in the type $A A$ action, the total multiplicity of the $\mathcal{A}(-\mathcal{Z})$ inputs (that is, the sum of multiplicities of $b_{1}^{\prime}, \ldots, b_{p}^{\prime}$ ) equals that of the $\mathcal{A}(\mathcal{Z})$ inputs (the sum of multiplicities of $b_{1}, \ldots, b_{q}$ ). From the definition using holomorphic curves, this is clear since each arrow comes from a domain in the standard Heegaard diagram of the identity diffeomorphism. We also note that $\widehat{\mathcal{A A}}\left(\mathbb{I}_{\mathcal{Z}}\right)$ can be given a refined relative grading where all generators have grading zero. 
The definition of the homotopy map $H$ involves first defining a specific ordering $<_{\mathcal{Z}}$ on the $4 g-1$ intervals of the pointed matched circle $\mathcal{Z}$. This means the determination of arrows is not local, in the sense that if we restrict to a certain interval of $\mathcal{Z}$, containing points paired outside the interval, then the type $A A$ arrows restricted to that interval may depend on how $\mathcal{Z}$ is configured outside the interval. However, we note that if all points are paired within the interval, then the ordering $<_{\mathcal{Z}}$ on these points (and therefore the type $A A$ arrows) is independent of outside configurations (this follows directly from how the ordering $<_{\mathcal{Z}}$ is defined). In particular, $\widehat{\mathcal{A A}}\left(\mathbb{I}_{\mathcal{Z}}\right)$ behaves well with respect to stabilization. That is, if $\stackrel{\circ}{\mathcal{Z}}=\mathcal{Z} \# \mathcal{Z}^{1}$, then $\widehat{\mathcal{A A}}\left(\mathbb{I}_{\mathcal{Z}}\right)$ is isomorphic to the appropriate restriction of $\widehat{\mathcal{A A}}\left(\mathbb{I}_{\mathcal{Z}}\right)$.

\section{D Certain arrows in $\widehat{\mathcal{D A}}$ of arcslides}

In this section we compute some of the arrows in $\widehat{\mathcal{D A}}(\tau)$ for a general arcslide $\tau$, using (16). From its description in the previous section, one can expect arrows in $\widehat{\mathcal{A A}}\left(\mathbb{I}_{\mathcal{Z}}\right)$ to be extremely complicated in general. The same would then be true for arrows in $\widehat{\mathcal{D A}}(\tau)$. We manage this complexity by focusing only on arrows whose algebra coefficients have a small total length (say length 1 or 2 on each side). It turns out that these are sufficient to prove the necessary properties of the box tensor products of $\widehat{\mathcal{D A}}\left(\tau_{i}\right)$ that we will need to consider.

Since the algebra coefficients have small total length, the domain corresponding to the arrow is supported in a small part of the Heegaard diagram. For arcslides, the parts of the Heegaard diagram that we are particularly interested in are the differences with the Heegaard diagram for the identity diffeomorphism, that is, around the points $b_{1}, c_{1}, c_{2}$ and $b_{1}^{\prime}$.

One source of complexity comes from the fact that the definition of the homotopy map $H$ in the construction of $\widehat{\mathcal{A A}}\left(\mathbb{I}_{\mathcal{Z}}\right)$ depends on the ordering $<_{\mathcal{Z}}$ on the intervals of the pointed matched circle. In a local situation, if we cannot tell which interval comes first in the ordering, we will need to cover all possible cases. Note that only the restriction of $<_{\mathcal{Z}}$ to the intervals covered by the algebra coefficients matter for determining the arrows.

When we show a set of local arrows in a given local situation and restriction of the ordering $<_{\mathcal{Z}}$, we intend to make the following assertions:

- There is an arrow for every way of extending the local arrow by completing the pointed matched circle and adding the appropriate number of horizontal lines to the algebra coefficients.

- Every arrow in the bimodule action whose algebra coefficients lie within the area shown can be obtained by extending one of the local arrows. 
We now begin with the simplest case: arrows in the type $A A$ action on $\widehat{\mathcal{A A}}\left(\mathbb{I}_{\mathcal{Z}}\right)$ where the algebra coefficients have length 1 on either side. The coefficients must then cover the same interval. The sequence of pairs $\left[a_{i, 1}, a_{i, 2}\right]$ is

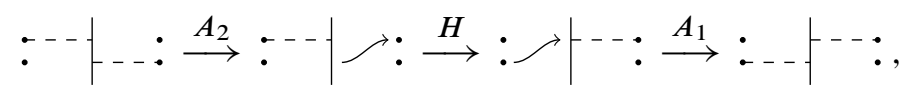

where the middle $H$ is [21, Case 3] of the homotopy map in the multiplicity-one case given there. This gives the arrow

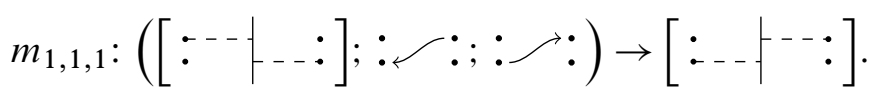

From (AA1), we obtain a simple method of deriving arrows in $\widehat{\mathcal{D A}}(\tau)$ from arrows in $\widehat{\mathcal{D D}}(\tau)$, in cases where the second coefficient of the type $D D$ arrow has length 1 (the second algebra action is the one that is involved in the box tensor product). For each type $D D$ arrow $\delta^{1}: \boldsymbol{x} \rightarrow a_{1} \otimes a_{2} \otimes \boldsymbol{y}$, where $a_{2}$ has length 1 , there corresponds a type $D A$ arrow $\delta_{2}^{1}:\left(\boldsymbol{x}, \bar{a}_{2}\right) \rightarrow a_{1} \otimes \boldsymbol{y}$, where by abuse of notation we use the same symbol to denote corresponding generators of $\widehat{\mathcal{D A}}(\tau)$ and $\widehat{\mathcal{D D}}(\tau)$.

As an application, we give a combinatorial proof of the following corollary.

Corollary 3.10 The tensor product $\widehat{\mathcal{A A}}\left(\mathbb{I}_{\mathcal{Z}}\right) \otimes \widehat{\mathcal{D D}}\left(\mathbb{I}_{\mathcal{Z}}\right)$ is homotopy equivalent to $\mathbb{I}$.

Proof Directly check each of the conditions in Lemma 3.8. For condition (ID-2), we use the refined relative grading on $\widehat{\mathcal{A A}}\left(\mathbb{I}_{\mathcal{Z}}\right)$ with all generators having grading zero. For condition (ID-3), use the type $A A$ arrows computed here. For (ID-4), use the stabilization property of $\widehat{\mathcal{A A}}\left(\mathbb{I}_{\mathcal{Z}}\right)$ discussed at the end of Section $3 \mathrm{C}$.

$3 D 1$ Type $\boldsymbol{A A}$ on a size- 2 interval: the disjoint pairs case The next simplest case for type $A A$ arrows is the size- 2 interval. First, we assume that no two of the three points are paired with each other. There are four subcases, depending on whether the middle idempotent is occupied on the left or on the right, and whether the lower or the upper interval comes first in the ordering $<_{\mathcal{Z}}$.

Case 1 The middle idempotent is on the left, the lower interval comes first in ordering. The only sequence covering the size- 2 interval is

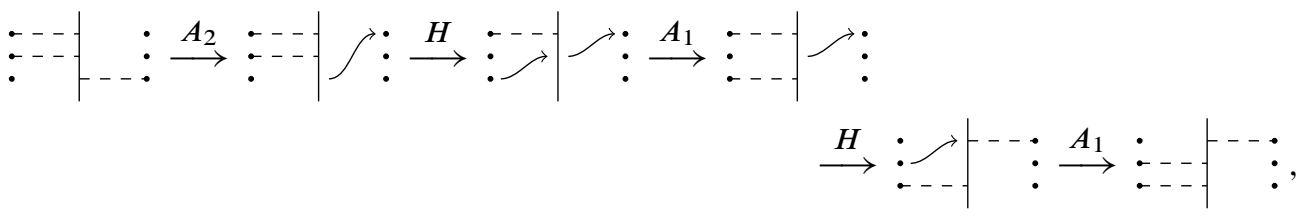


giving the arrow

(AA2)

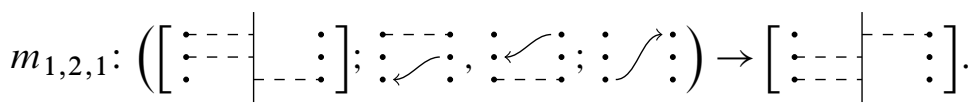

Note that in the first $H$-move, we shift only the lower part of the strand to the left, since the lower interval comes first in the ordering.

Case 2 The middle is idempotent on the left, the upper interval comes first in ordering. The only sequence covering the size- 2 interval is

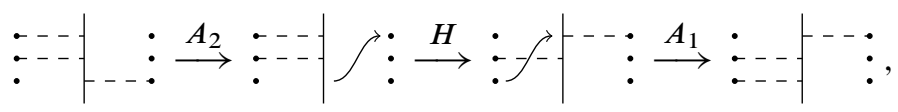

giving the arrow

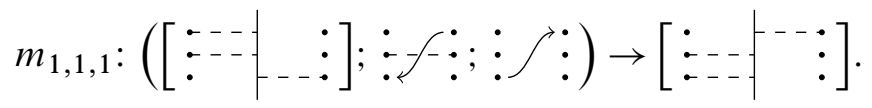

Here the upper interval comes first, so we shift the entire strand to the left in the first $H$-move.

Case 3 The middle idempotent is on the right, the upper interval comes first in ordering. In this case there are two possible sequences covering the size- 2 interval. The first one is

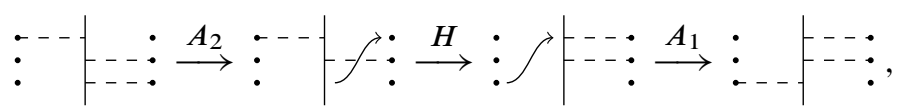

giving the arrow

(AA4)

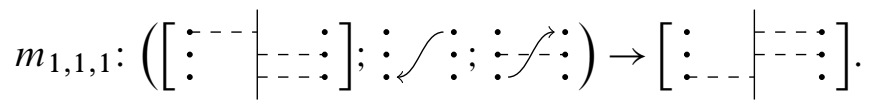

The second one is

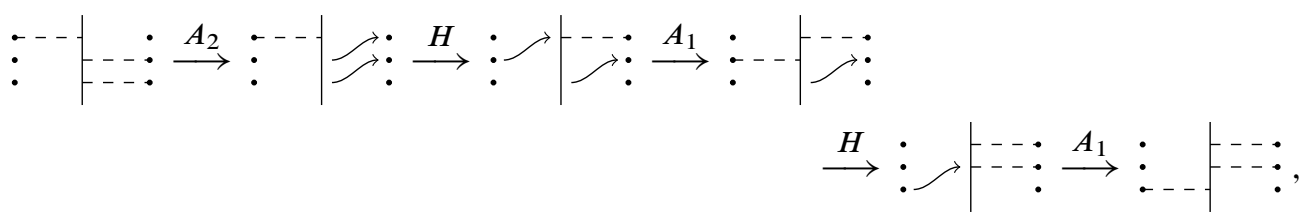

giving the arrow

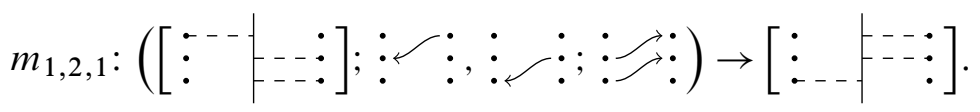


Case 4 The middle idempotent is on the right, the lower interval comes first in ordering. The only sequence covering the size- 2 interval is
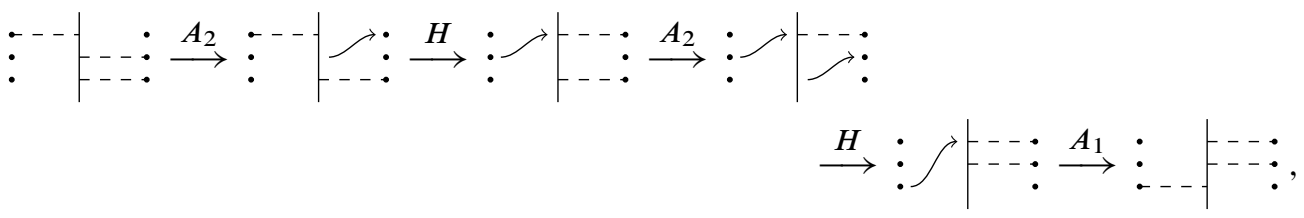

giving the arrow

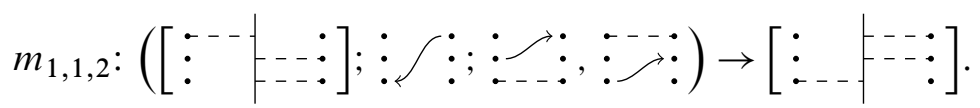

As examples, we show the computation of type $D A$ arrows in $\widehat{\mathcal{A A}}\left(\mathbb{I}_{\mathcal{Z}}\right) \otimes \widehat{\mathcal{D D}}\left(\mathbb{I}_{\mathcal{Z}}\right)$ that cover a size- 2 interval. While the results in the remainder of this section will not be used directly in what follows, it serves as a model for the calculations of similar arrows in $\widehat{\mathcal{D A}}(\tau)$ for an arcslide $\tau$.

To compute the type $D A$ arrows, we combine the previous results with what is known about type $D D$ arrows in $\widehat{\mathcal{D D}}\left(\mathbb{I}_{\mathcal{Z}}\right)$. On the size- 2 interval, the possibilities are given below (on each line, $\delta^{1}: \boldsymbol{x} \rightarrow\left(a, a^{\prime}\right) \otimes \boldsymbol{y}$ represents the arrow $\delta^{1}: \boldsymbol{x} \rightarrow a \otimes a^{\prime} \otimes \boldsymbol{y}$, where $a \in \mathcal{A}(\mathcal{Z})$ and $a^{\prime} \in \mathcal{A}(-\mathcal{Z})$ ):

(DD1)

(DD2)

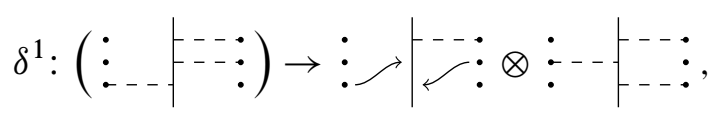

(DD3)

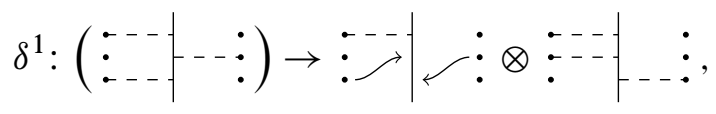

(DD4)

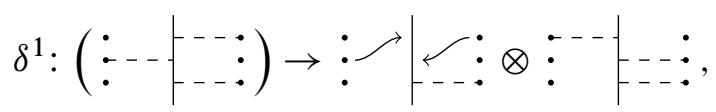

(DD5)

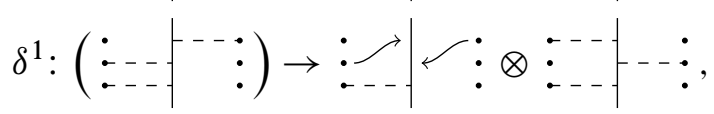

(DD6)

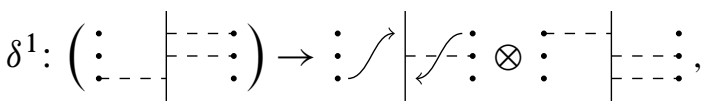

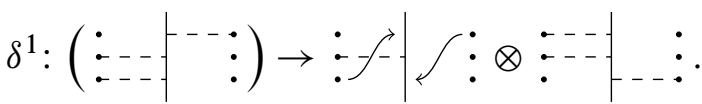

It is now a matter of combining these following the rules of the box tensor product. In the figures below, for both type $D D$ and type $A A$ bimodules, we will show the first algebra action on the left and the second algebra action on the right. This is purely for ease of visualization, and does not indicate which side the algebras act on. Indeed, both actions on the type $D D$ bimodule are on the left, and both actions on the type $A A$ bimodule are on the right. Nevertheless, we will often talk about left action or left 


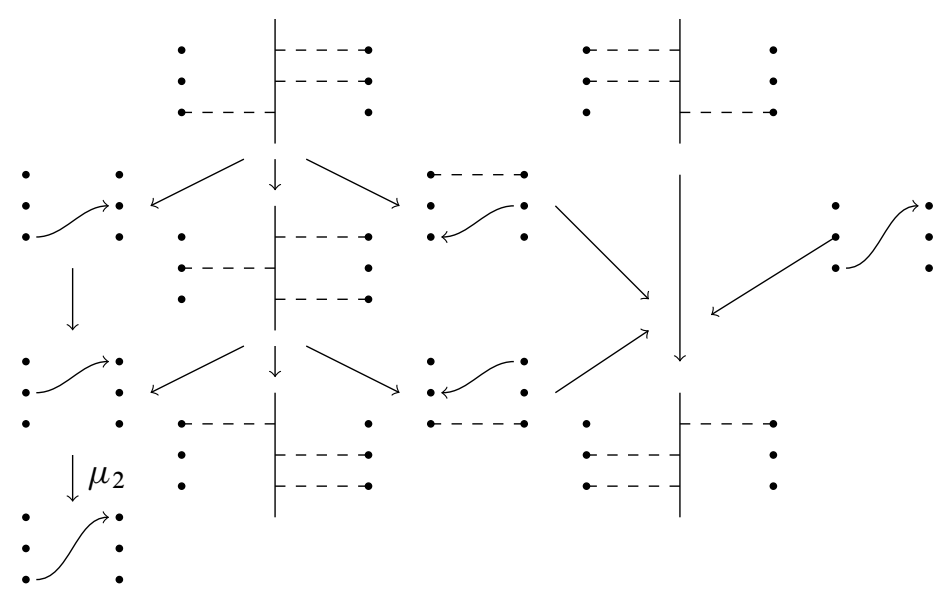

Figure 6: Formation of type $D A$ operation, case 1

idempotents to match how the figures are drawn. Moreover, we will put the $D D$ arrows on the left, and $A A$ arrows on the right, since we are tensoring the second action in $\widehat{\mathcal{D D}}\left(\mathbb{I}_{\mathcal{Z}}\right)$ with the first action in $\widehat{\mathcal{A A}}\left(\mathbb{I}_{\mathcal{Z}}\right)$.

Each type $D A$ arrow comes from a single type $A A$ arrow and zero or more type $D D$ arrows. The left outputs (in $\mathcal{A}(\mathcal{Z})$ ) of the type $D D$ arrows are multiplied together to give the overall type $D$ output, while the right outputs (in $\mathcal{A}(-\mathcal{Z})$ ) are given as the left inputs to the type $A A$ arrow. The overall type $A$ inputs in $\mathcal{A}(\mathcal{Z})$ are given as the right inputs to the type $A A$ arrow.

The right idempotent of the $D D$ generator must agree with the left idempotent of the $A A$ generator. The left idempotent of the $D D$ generator and the right idempotent of the $A A$ generator then combine to form the idempotent of the resulting $D A$ generator.

We now look at each of the four cases.

Case 1 The middle idempotent is on the left, the lower interval comes first in ordering. This combination is shown in Figure 6. We use (DD1), (DD3), and (AA2). The resulting arrow is

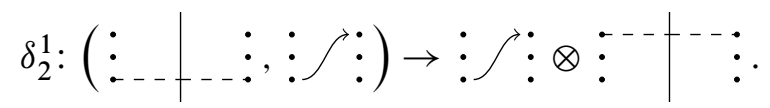

Case 2 The middle idempotent is on the left, the upper interval comes first in ordering; see Figure 7 We use (DD5) and (AA3). The resulting arrow is the same as in (DA1), so in this case the order of the two intervals already does not matter at the $D A$ level. 


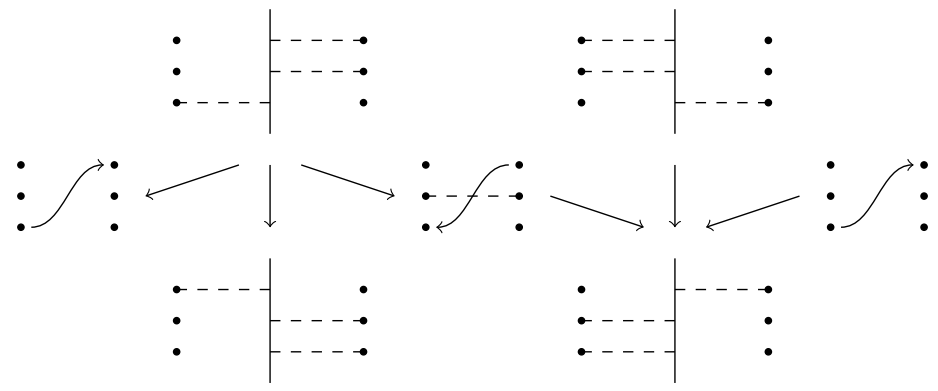

Figure 7: Formation of type $D A$ operation, case 2

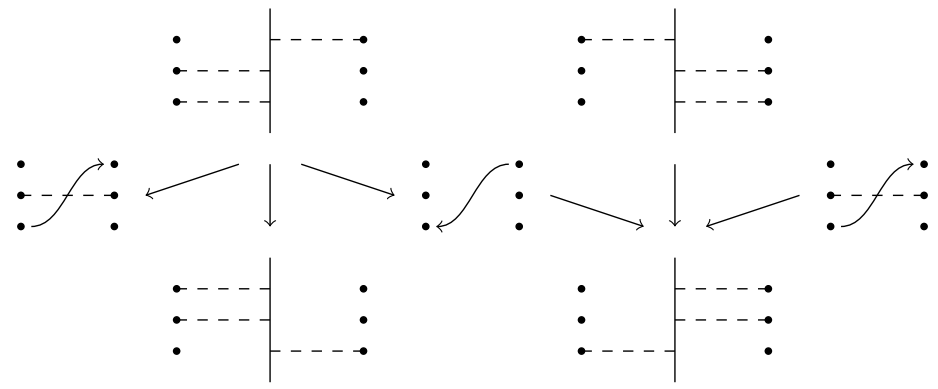

Figure 8: Formation of type $D A$ operation, case 3

Case 3 The middle idempotent is on the right, the upper interval comes first in ordering; see Figure 8. We use (DD6) and (AA4), and the resulting arrow is

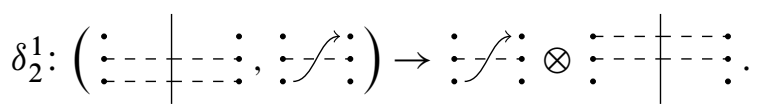

Another combination, using (DD4), (DD2), and (AA5), gives the arrow

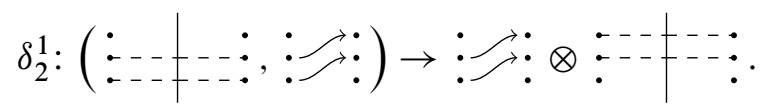

Case 4 The middle idempotent is on the right, the lower interval comes first in ordering, shown in Figure 9. We use (DD6) and (AA6), and the resulting arrow is

(DA4)

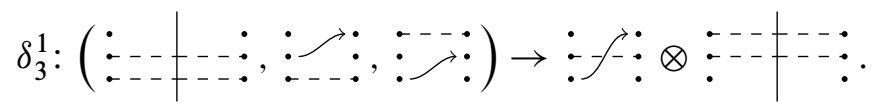

This arrow shows that the model $\widehat{\mathcal{A A}}\left(\mathbb{I}_{\mathcal{Z}}\right) \otimes \widehat{\mathcal{D D}}\left(\mathbb{I}_{\mathcal{Z}}\right)$ of $\widehat{\mathrm{CFDA}}\left(\mathbb{I}_{\mathcal{Z}}\right)$ is not exactly the same, but only homotopy equivalent to $\mathbb{I}$. 


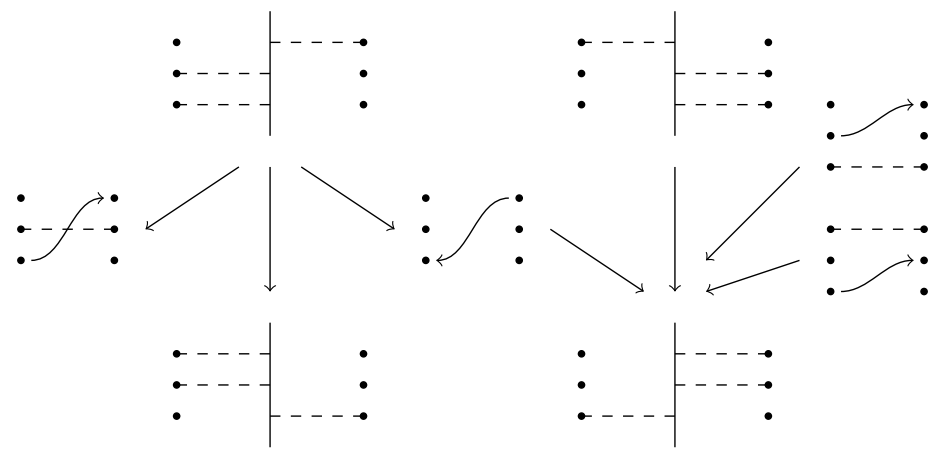

Figure 9: Formation of type $D A$ operation, case 4

$3 D 2$ Pieces of arcslide We now compute some simple arrows in $\widehat{\mathcal{D A}}(\tau)$ for an arcslide $\tau$. Since the method used here is similar to that in the previous section, we will show only the results.

First, we consider the case where $b_{1}$ is directly above $c_{1}$, and compute the arrows in $\widehat{\mathcal{D A}}(\tau)$ corresponding to the region of the Heegaard diagram around $b_{1}$. The Heegaard diagram around $b_{1}$ is this:

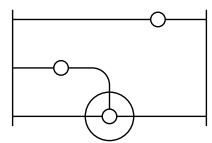

The possible type $D D$ arrows are the following:

(DD7)

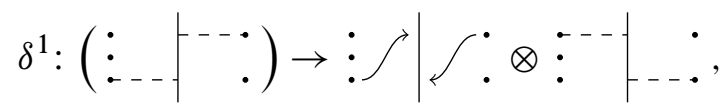

(DD8)

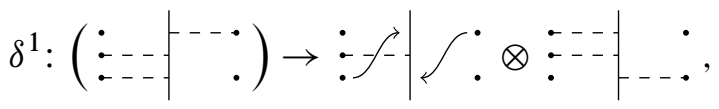

(DD9)

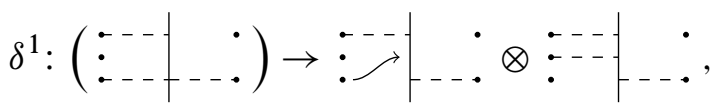

(DD10)

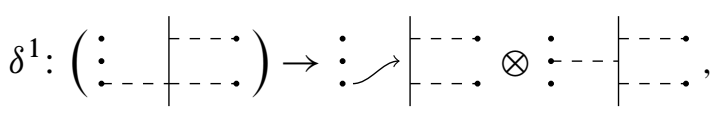

(DD11)

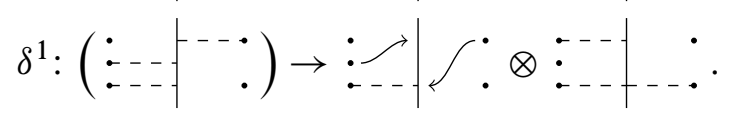

This comes directly from [11]. The only potentially tricky part is figuring out the possible locations of idempotents. For example, in the arrow $\delta^{1}: \boldsymbol{x} \rightarrow(a(\sigma), 1) \otimes \boldsymbol{y}$ (third and fourth arrows above; $\sigma$ is the chord $c_{1} \rightarrow b_{1}$ ), the left idempotent of $\boldsymbol{y}$ must be occupied at the $B$ pair and unoccupied at the $C$ pair. Since generators of $\widehat{\mathcal{D D}}(\tau)$ either have complementary idempotents or idempotents that are both occupied at $C$, 
the right idempotent of $\boldsymbol{y}$ must be occupied at $C$ (so $\boldsymbol{y}$ is of type $X$ ). From the idempotent of $\boldsymbol{y}$, we can deduce that of $\boldsymbol{x}$, and see that $\boldsymbol{x}$ is of type $Y$. Similar arguments are used to list possible idempotents in the other cases.

Computing the type $D A$ arrows in this case is relatively straightforward, as we are combining with the arrow (AA1) on a size- 1 interval. The results are as follows, where (DA5)-(DA9) follow respectively from (DD7)-(DD11):

(DA6)

(DA7)

(DA8)

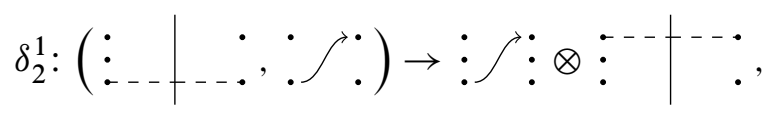

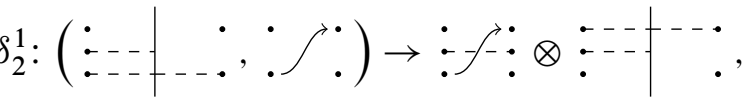

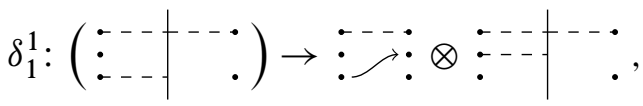

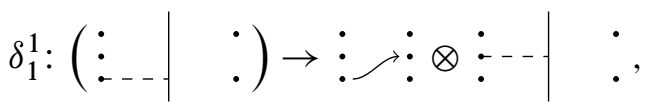

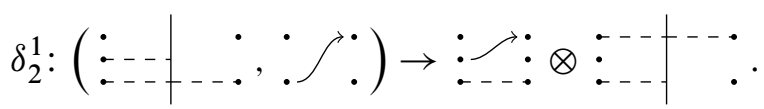

Now we consider other side of the same case, computing arrows in $\widehat{\mathcal{D A}}(\tau)$ corresponding to the region around $b_{1}^{\prime}$. Since $b_{1}$ is directly above $c_{1}$, we have $b_{1}^{\prime}$ directly below $c_{2}$, and the Heegaard diagram around $b_{1}^{\prime}$ is this:

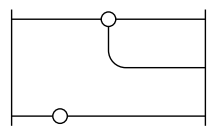

The type $D D$ operations are these:

(DD12)

(DD13)

(DD14)

(DD15)

(DD16)

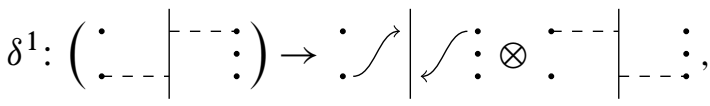

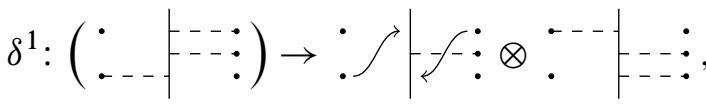

$$
\begin{aligned}
& \delta^{1}:\left(\begin{array}{c|c|c|}
\cdots & - \\
\cdots
\end{array}\right) \rightarrow \cdots \vdots \otimes \ldots,
\end{aligned}
$$

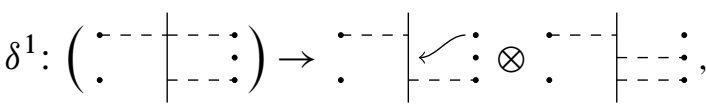

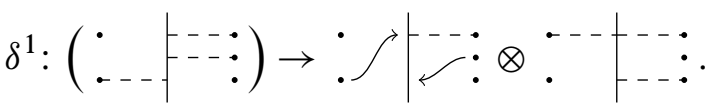


This time we will need to combine with type $A A$ arrows on a size- 2 interval, emulating the method in Section 3D1. The results are the following:

(DA10)

(DA11)

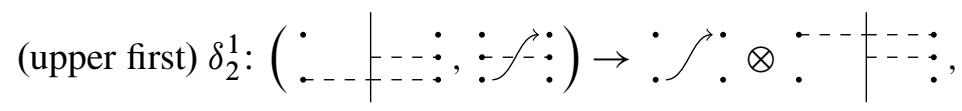

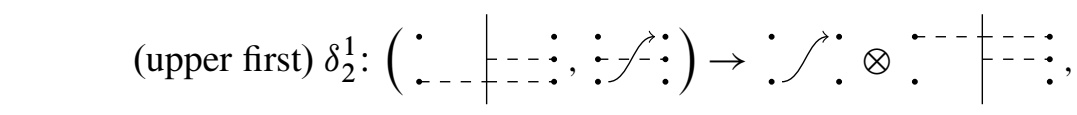

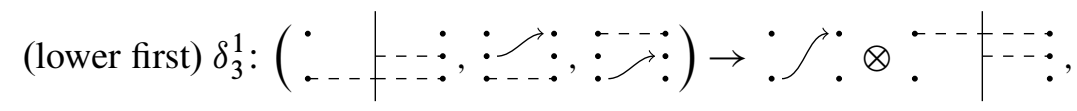

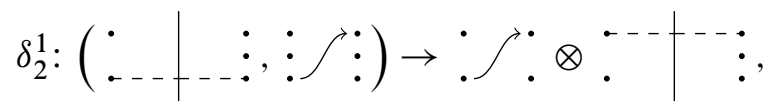

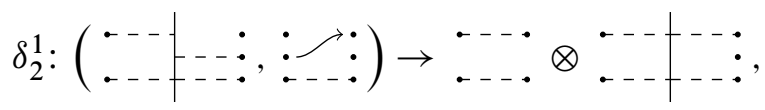

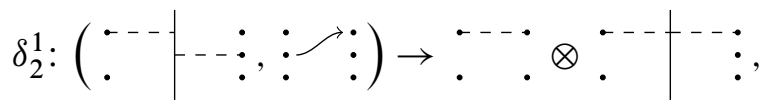

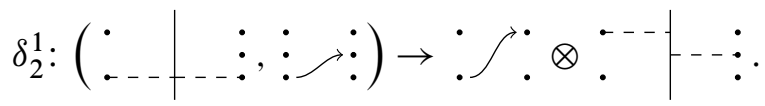

The first arrow follows from (DD12) and (AA4) only if the upper interval comes first in the ordering $<_{\mathcal{Z}^{\prime}}$ for the right pointed matched circle. The second arrow follows from (DD12) and (AA6) only if the lower interval comes first in the ordering. The third arrow does not depend on ordering. However, it is formed in different ways for the two orderings: if upper interval comes first, it follows from (DD13) and (AA3); otherwise it follows (DD16), (DD15), and (AA2). The last three arrows are independent of ordering. They follow from (AA1) and respectively (DD14)-(DD16).

The cases where $b_{1}$ is directly below $c_{1}$ (and therefore $b_{1}^{\prime}$ is directly above $c_{2}$ ) are very similar. Here is the Heegaard diagram around $b_{1}$ :

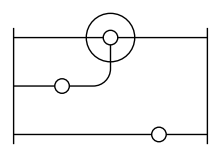

The first two DA arrows are the same as (DA5) and (DA6), and the last three are modified appropriately from (DA7)-(DA9). The Heegaard diagram around $b_{1}^{\prime}$ is this:

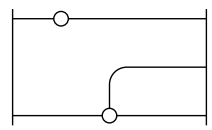

The first three $D A$ arrows are the same as arrows are modified appropriately from (DA13)-(DA15). 
3D3 Type $\boldsymbol{A A}$ on a size- 2 interval: the paired case We now consider the case of a size- 2 interval, where the top and bottom points are paired with each other. Here the lower interval immediately precedes the upper interval in the ordering $<_{\mathcal{Z}}$. There are no arrows starting at generators where the middle idempotent is on the same side as the idempotent containing the top and bottom points. Starting at generators where the middle idempotent is to the right, there is a sequence

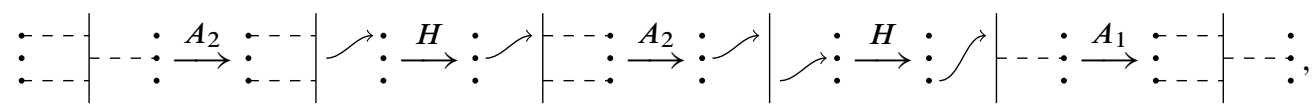

giving the arrow

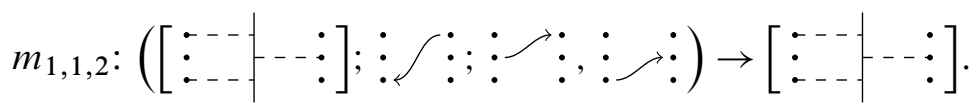

Starting at generators where the middle idempotent is to the left, there is a sequence

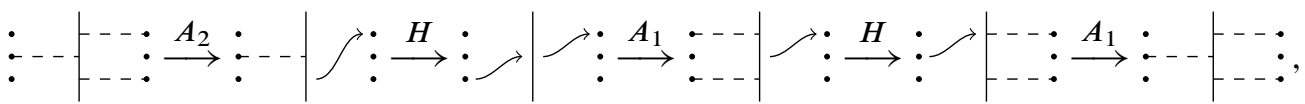

giving the arrow

(AA8)

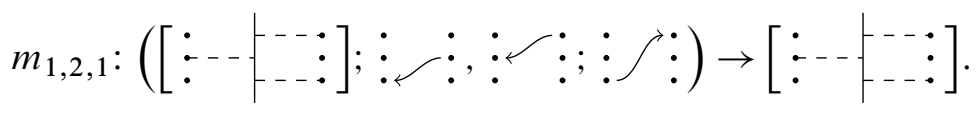

Furthermore, there are several infinite series of arrows formed by repeating the moves used above. We list the two arrows that will be used later in the paper:

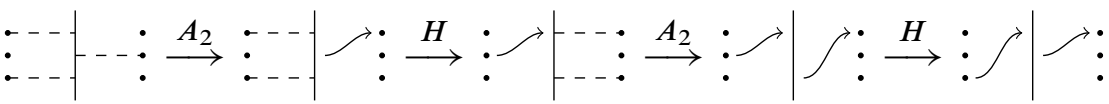

gives the arrow

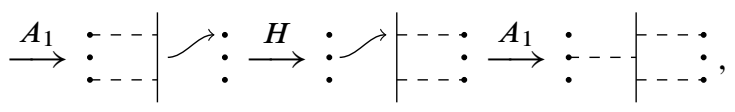

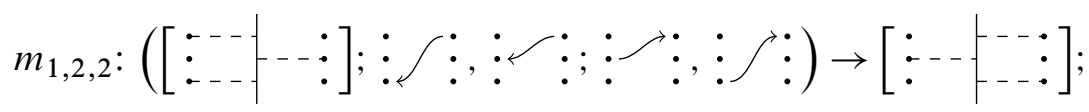

and

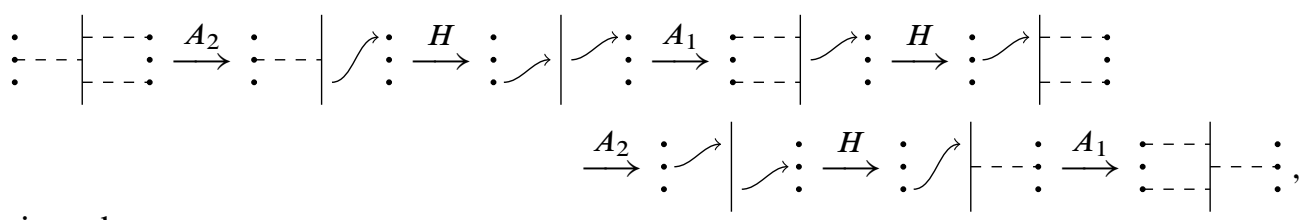

gives the arrow

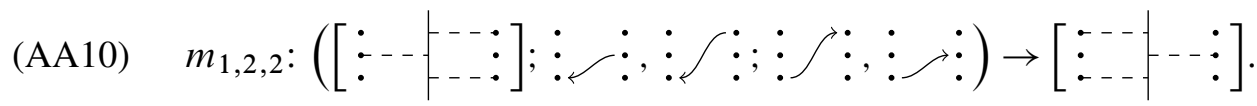


Note that in the derivation of the first arrow, we used [21, Case 3] of the homotopy map $H$ in the multiplicity greater than one case. From this we see that the type $A A$ bimodule can have infinitely many arrows. However, in our examples, only a finite number of them will be used when constructing the action on type $D A$ invariants.

$3 D 4$ Short underslide Using results from the previous section, we compute type $D A$ arrows for the short underslide. These are underslides where $b_{1}$ is the only point between $c_{1}$ and $c_{2}$. Hence $b_{1}$ and $b_{1}^{\prime}$ are located in the same region of the Heegaard diagram, which is the only region of interest. Here is the diagram for the case where $b_{1}$ is directly above $c_{1}$ :

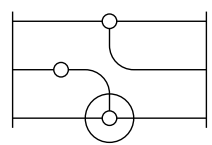

The possible type $D D$ arrows are the following:

(DD17)

(DD18)

(DD19)

(DD20)

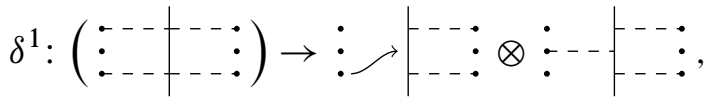

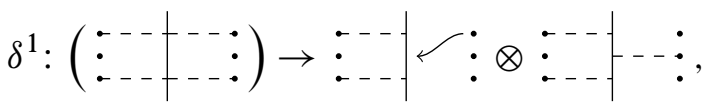

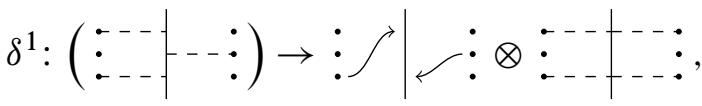

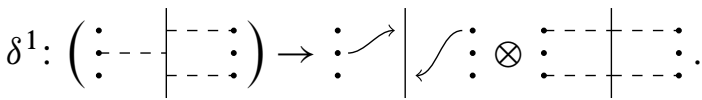

These give rise to type $D A$ arrows

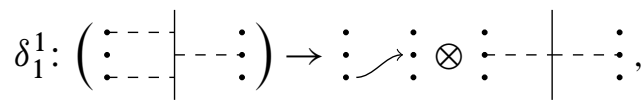

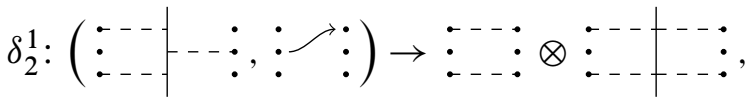

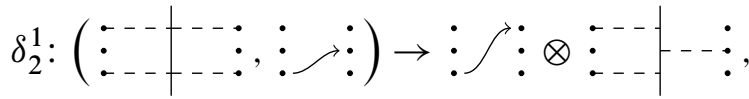

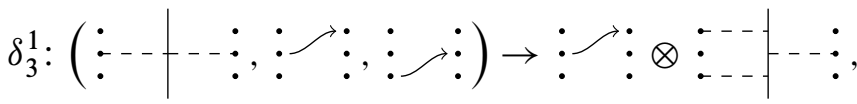

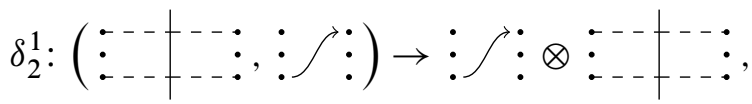

$$
\begin{aligned}
& \delta_{3}^{1}:(\vdots-\cdots, \vdots \vdots, \vdots \vdots) \rightarrow \vdots \vdots \otimes \vdots+\cdots
\end{aligned}
$$


Here arrows (DA16)-(DA18) follow respectively from (DD17)-(DD19). Arrow (DA19) follows from (DD20) and (AA7). Arrow (DA20) follows from (DD19), (DD18), and (AA8). Arrow (DA21) follows from (DD20), (DD18), and (AA9).

The diagram for the case where $b_{1}$ is directly below $c_{1}$ is this:

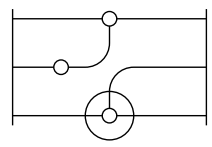

The possible type $D D$ arrows are the following:

(DD21)

(DD22)

(DD23)

(DD24)

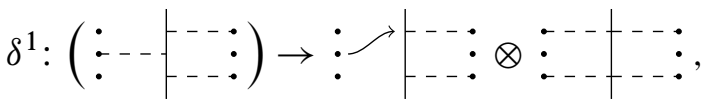

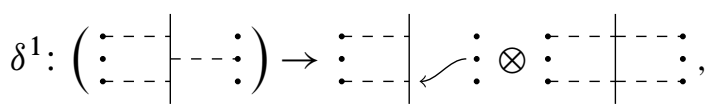

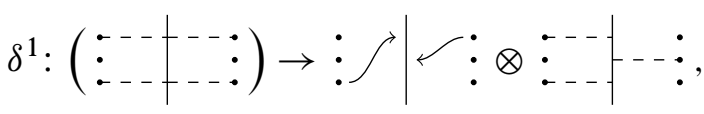

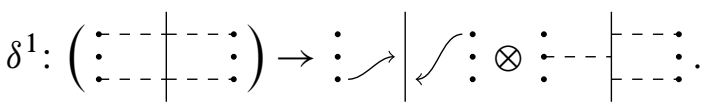

These give rise to type $D A$ arrows:

(DA22)

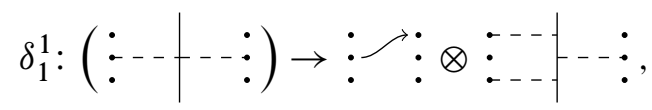

(DA23)

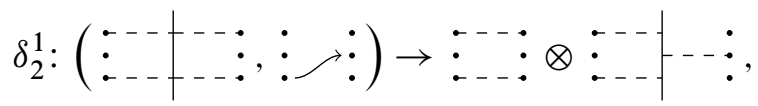

(DA24)

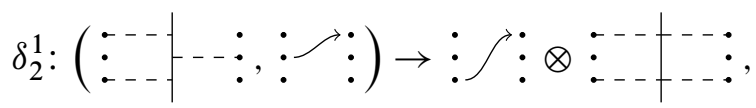

(DA25)

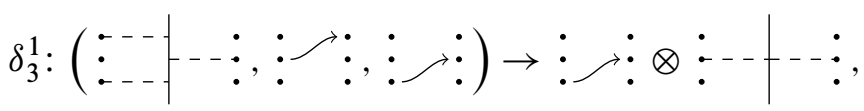

(DA26)

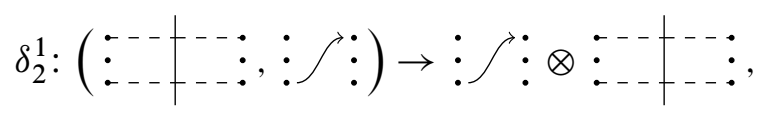

(DA27)

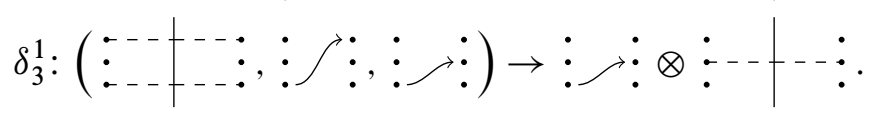

Here arrows (DA22)-(DA24) follow respectively from (DD21)-(DD23). Arrow (DA25) follows from (DD24) and (AA7). Arrow (DA26) follows from (DD22), (DD23), and (AA8). Arrow (DA27) follows from (DD22), (DD24), and (AA10). 
3D5 Type $\boldsymbol{A A}$ on two separated intervals with pairing To consider more local situations for the arcslide, we will need type $A A$ arrows on two separated intervals, such that either the two inner positions or the two outer positions are paired. These two cases are very similar, so we will only write out the first case here.

In this case, the upper interval immediately precedes the lower interval in the ordering $<_{\mathcal{Z}}$. If the middle idempotent (consisting of the two paired inner points) is occupied on the left, then it is not possible to multiply both intervals to the right as the first step. So the only possible sequence of $\left[a_{i, 1}, a_{i, 2}\right]$ is the following:

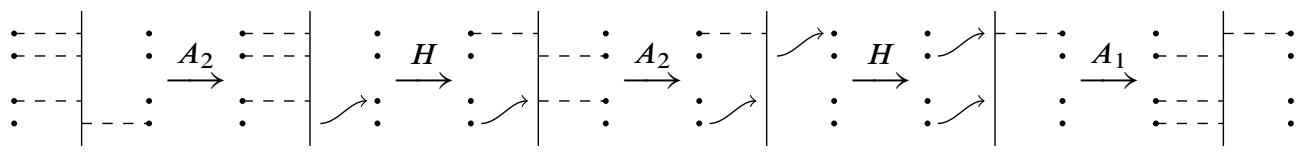

giving the arrow

(AA11)

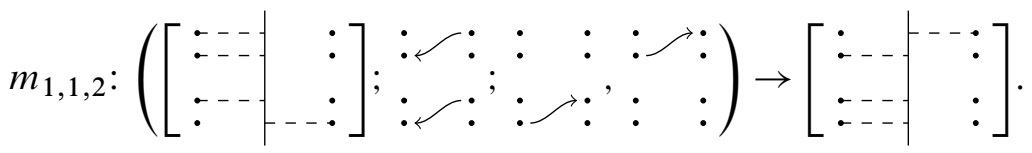

In these diagrams, the two middle positions are paired, and there can be an arbitrary number of points between them in the full pointed matched circle. Since no arrow in $\widehat{\mathcal{D D}}\left(\mathbb{I}_{\mathcal{Z}}\right)$ gives off an algebra element with two separate strands, this cannot be used to form a type $D A$ arrow for the identity.

If the middle idempotent is occupied on the right, it is possible to multiply both intervals to the right as the first step, but not possible to multiply only the lower interval. So the only sequence is

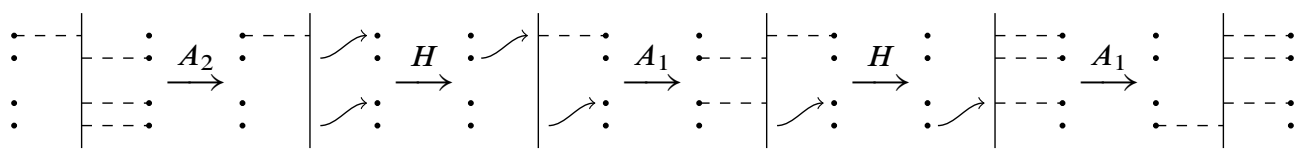

giving the arrow

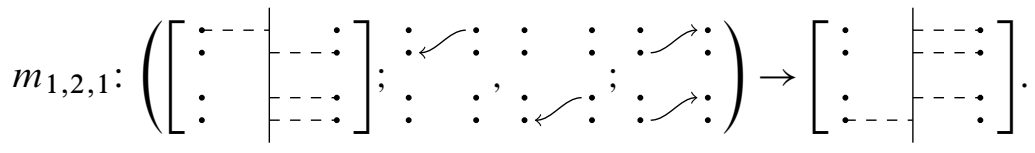

This leads to the following type $D A$ arrow for identity:

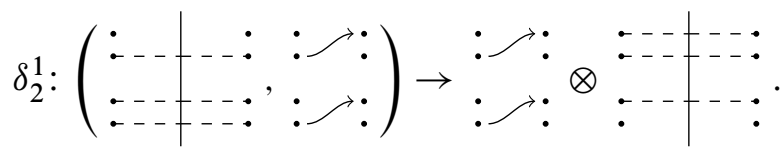


3D6 More pieces of arcslide We now compute some arrows whose corresponding domains touch both $c_{1}$ and $c_{2}$. We focus on the overslide cases; the underslide cases are similar. First, if $b_{1}$ is directly above $c_{1}$, the local Heegaard diagram is as follows. We focus on arrows whose domain is restricted inside this diagram:

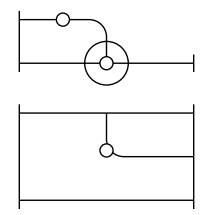

The two horizontal lines where the 1 -handle is attached contain the $\alpha$-arcs for the $C$ pair. Immediately above and below are the points $b_{1}$ on the left and $b_{1}^{\prime}$ on the right. For clarity, we list all type $D D$ and $D A$ arrows in this region, even though some may already have been covered in previous cases. These are the type $D D$ arrows:

(DD25)

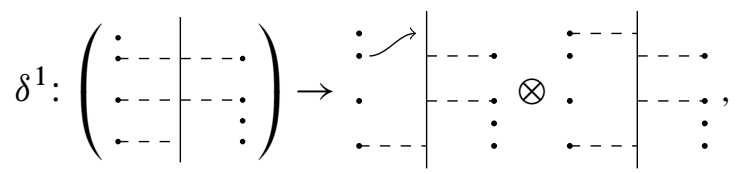

(DD26)

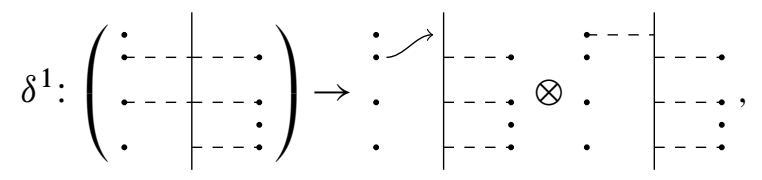

(DD27)

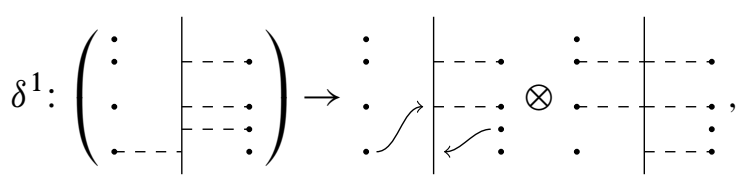

(DD28)

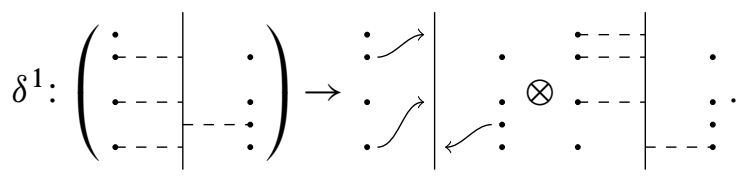

These give rise to the following type DA arrows. Here (DA29)-(DA32) follow respectively from (DD25)-(DD28):

(DA29)

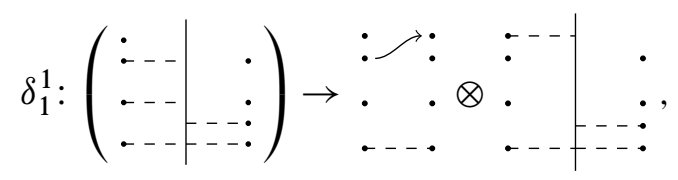

(DA30)

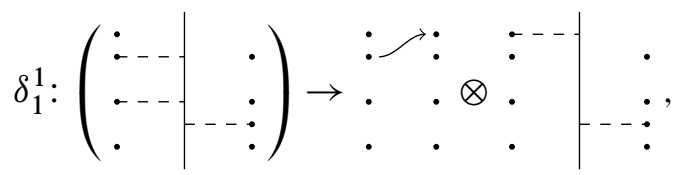


(DA31)

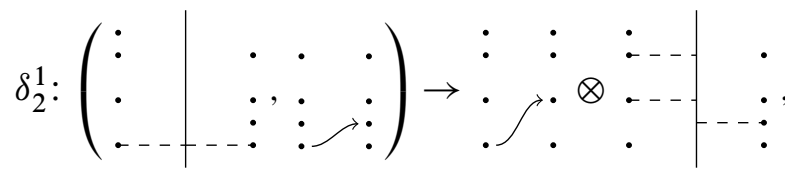

(DA32)

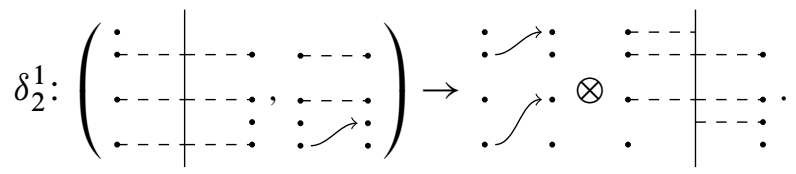

The case where $b_{1}$ is directly below $c_{1}$ is again more complicated. Here is the local Heegaard diagram:

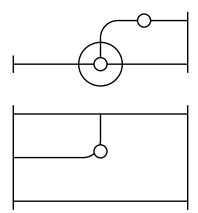

The type $D D$ arrows are these:

(DD29)

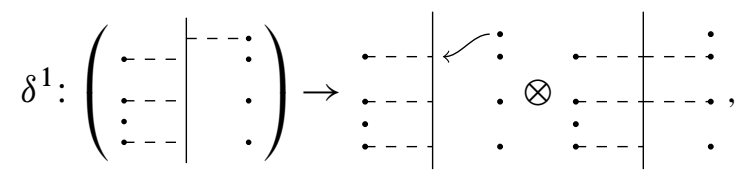

(DD30)

(DD31)

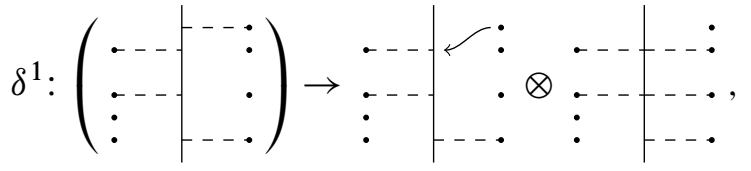

(DD32)

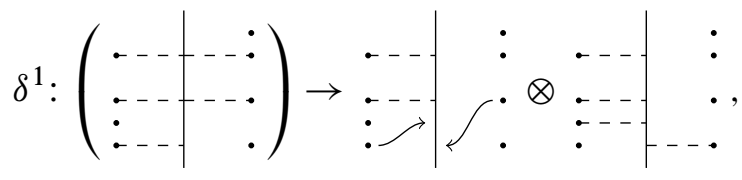

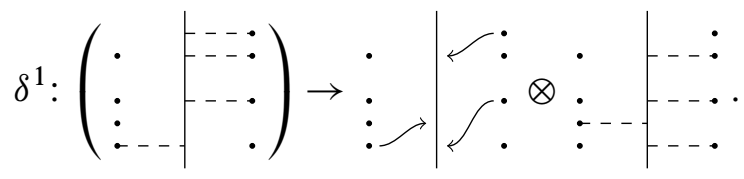

The resulting type $D A$ arrows are the following:

(DA33)

(DA34)

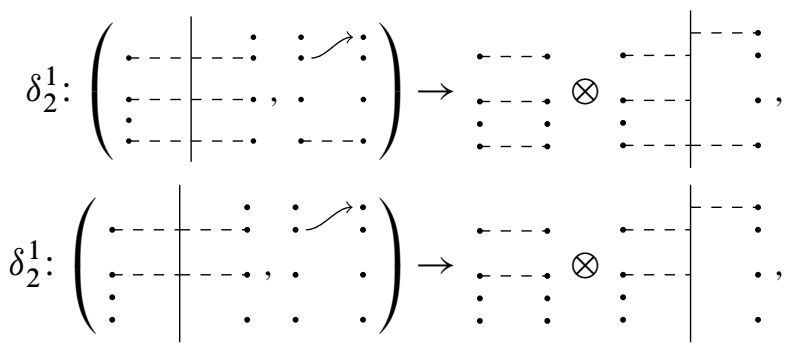


(DA35)

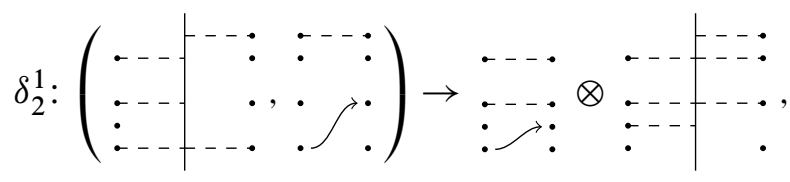

(DA36)

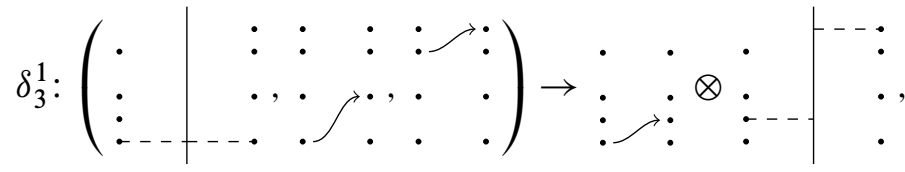

(DA37)

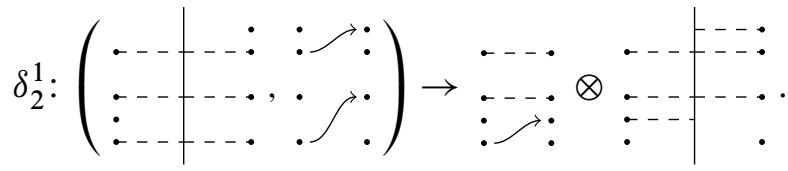

Here, arrows (DA33)-(DA35) follow respectively from (DD29)-(DD31). Arrow (DA36) follows from (DD32) and (AA11). Arrow (DA37) follows from (DD29), (DD31), and (AA12).

\section{Relations on the mapping class groupoid}

In this section, we conclude the proof of Theorem 2.2. In Section 4A, we describe how to enumerate the set of generators of a box tensor product of $\widehat{\mathcal{D A}}\left(\tau_{i}\right)$, where $\tau_{i}$ are arcslides, and how properties of $\widehat{\mathcal{D D}}\left(\tau_{i}\right)$ carry over to properties of $\widehat{\mathcal{D A}}\left(\tau_{i}\right)$ and their box tensor products. With all these preparations in place, we prove (17) for the involution relation in Section $4 \mathrm{~B}$, and for the other relations in Section 4C.

\section{A Compositions of arcslides}

Given an arcslide $\tau$, the description of the set of generators of $\widehat{\mathcal{D A}}(\tau)$ follows from that of $\widehat{\mathcal{D D}}(\tau)$ and $\widehat{\mathcal{A A}}\left(\mathbb{I}_{\mathcal{Z}}\right)$. The generators are classified by their idempotents on the two sides (type $D$ idempotent on the left and type $A$ idempotent on the right). As before, we use the canonical identification of pairs of points between the pointed matched circles on the two sides. There are two types of generators in $\widehat{\mathcal{D A}}(\tau)$ :

- Type $X$ The idempotents on the two sides contain the same pairs.

- Type $Y$ The idempotents on the two sides differ at exactly one pair, with the $C$ pair occupied on the left and $B$ pair occupied on the right.

Using this, and the definition of box tensor product, we can enumerate the set of generators of

$$
\widehat{\mathcal{D A}}\left(\tau_{1}\right) \otimes \cdots \otimes \widehat{\mathcal{D A}}\left(\tau_{n}\right)
$$

for a sequence of arcslides $\tau_{1}, \ldots, \tau_{n}$. We now describe the procedure in detail. 
First, we combine the identification between pairs of points on the starting and ending pointed matched circles of a single arcslide to obtain an identification of pairs on all pointed matched circles appearing in the sequence. Note that even if the starting and ending pointed matched circle of a sequence is the same, the identification of pairs between the two, induced by the sequence of arcslides, may not be the identity. See the triangle relation for an example.

With this identification of pairs throughout a sequence, we can talk about a pair of points in the sequence. These are pairs of points, one for each pointed matched circle in the sequence, that are identified to be the same. We assign a number from 1 to $d$ to each pair of points in the sequence that served as either the $B$ pair or the $C$ pair of some arcslide, where $d$ is the total number of such pairs.

Each generator of the box tensor product $\widehat{\mathcal{D A}}\left(\tau_{1}\right) \otimes \cdots \otimes \widehat{\mathcal{D A}}\left(\tau_{n}\right)$ is of the form $\boldsymbol{x}_{1} \otimes \cdots \otimes \boldsymbol{x}_{n}$, where $\boldsymbol{x}_{i}$ is a generator of $\widehat{\mathcal{D A}}\left(\tau_{i}\right)$ for each $1 \leq i \leq n$, and the right idempotent of $\boldsymbol{x}_{i}$ agrees with the left idempotent of $\boldsymbol{x}_{i+1}$ for each $1 \leq i<n$. A generator $x_{1} \otimes \cdots \otimes x_{n}$ is determined by the set of occupied pairs at the starting and ending pointed matched circles, and at each pointed matched circles in the middle. It is clear that each unnumbered pair must be either occupied throughout or unoccupied throughout. For the numbered pairs, the only possible changes are as follows: suppose that for a certain arcslide $\tau_{i}$ in the sequence, the $B$ pair is numbered $b_{i}$ and the $C$ pair is numbered $c_{i}$; then it is possible to have $c_{i}$, but not $b_{i}$, occupied in the left idempotent of $\boldsymbol{x}_{i}$, and $b_{i}$, but not $c_{i}$, occupied in the right idempotent, with all other pairs staying the same. This corresponds to choosing $\boldsymbol{x}_{i}$ to have type $Y$.

We can therefore specify a type of generators by specifying which of the numbered pairs are occupied at each pointed matched circle. At each arcslide, the generator is either type $X$ or type $Y$. In the first case, the occupied pairs must be the same before and after, and in the second case, the $C$ pair occurs before and is replaced by the $B$ pair. To choose a specific generator of a given type, it remains to choose which unnumbered pairs to occupy throughout, so that the total number of occupied pairs is $g$ (half of the total $2 g$ pairs).

We now study the involution relation as an example. This is the simplest case, which nevertheless illustrates most of the reasoning required. One possible Heegaard diagram for the involution relation is shown in Figure 10. There are two numbered pairs, that is, $d=2$. Pair 1 served as the $C$ pair and pair 2 served as the $B$ pair for both arcslides. The possible types of generators are
() $X_{()} X_{(),(1)} X_{(1)} X_{(1)}$,
(2) $X$
$X_{(2)} X_{(2)}$
(12) $X_{(12)}$
$X_{(12)} X_{(12)}$
$X_{(12)}$
(1) $X_{(1)} Y_{(2)}$
$Y_{(2)},{ }_{(1)} Y_{(2)} X_{(2)}$ 


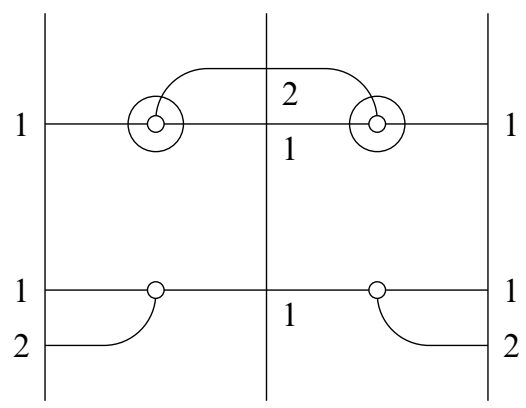

Figure 10: Heegaard diagram for the involution relation

For generators with $X$ at both positions, any combination of occupying pairs is possible. For generators with $X$ at first position and $Y$ at second position, pair 1 and not pair 2 are occupied in the middle, so 1 can be replaced by 2 at the end. This implies pair 1 and not pair 2 are occupied at the beginning, as well. The same reasoning can be used for type $Y X$, and to show that type $Y Y$ is not possible.

Later, we may use $(*)$ to denote an arbitrary subset of the numbered pairs, that stays the same for a given generator. So for example, we may collect the first four types above into $(*) X_{(*)} X_{(*)}$.

We now state several general facts about $\widehat{\mathcal{D A}}(\tau)$ and the box tensor products of such bimodules. These follow from the corresponding facts about $\widehat{\mathcal{D D}}(\tau)$ in Section $2 \mathrm{E}$, and the definition of $\widehat{\mathcal{D A}}(\tau)$ as $\widehat{\mathcal{A A}}\left(\mathbb{I}_{\mathcal{Z}}\right) \otimes \widehat{\mathcal{D D}}(\tau)$.

Remark 4.1 (relation with Heegaard diagram) Just as in the type $D D$ case, each generator of $\widehat{\mathcal{D A}}(\tau)$ corresponds to a tuple of points in the standard Heegaard diagram for the arcslide, with its left (type $D$ ) idempotent the set of unoccupied $\alpha$-arcs on the left, and its right (type $A$ ) idempotent the set of occupied $\alpha$-arcs on the right. When Heegaard diagrams of arcslides are glued side-by-side along their boundaries, the result is a larger Heegaard diagram that now contains $\alpha$-circles. Note that the boundaries that are glued along are removed from the resulting diagram. Each generator $\boldsymbol{x}_{1} \otimes \cdots \otimes \boldsymbol{x}_{n}$ of the box tensor product corresponds to a tuple of points, with each $\alpha$ and $\beta$ circles containing exactly one point, and each $\alpha$-arc containing at most one point.

As in the type $D D$ case, each arrow in $\widehat{\mathcal{D A}}(\tau)$ corresponds to a domain away from the basepoint in the Heegaard diagram of the arcslide. Likewise, each arrow in the box tensor product $\widehat{\mathcal{D A}}\left(\tau_{1}\right) \otimes \cdots \otimes \widehat{\mathcal{D A}}\left(\tau_{n}\right)$ corresponds to a domain in the Heegaard diagram obtained by gluing the diagrams for $\tau_{1}, \ldots, \tau_{n}$ in sequence. The multiplicity of the domain on the left (resp. right) boundary equals the total multiplicity of the algebra coefficients on the left (resp. right) of the arrow. The relation $\partial\left(\partial^{\alpha} B\right)=\boldsymbol{y}-\boldsymbol{x}$, when domain $B$ represents an arrow from $\boldsymbol{x}$ to $\boldsymbol{y}$, still holds. 
Remark 4.2 (grading) The remarks on the grading set of $\widehat{\mathcal{D D}}(\tau)$ for an arcslide $\tau$ extends to a similar statement for $\widehat{\mathcal{D A}}(\tau)$, and by taking box tensor products, extends to $\widehat{\mathcal{D A}}(\phi, \tau)$. Given $\phi: F^{\circ}\left(\mathcal{Z}_{1}\right) \rightarrow F^{\circ}\left(\mathcal{Z}_{2}\right)$ and a factorization $\tau$ of $\phi$, the bimodule $\widehat{\mathcal{D A}}(\phi, \tau)$ is graded by a set $S$ with free and transitive left-right actions by $G\left(\mathcal{Z}_{1}\right)$ and $G\left(\mathcal{Z}_{2}\right)$. The grading set induces an element of $\operatorname{Out}\left(G\left(\mathcal{Z}_{1}\right), G\left(\mathcal{Z}_{2}\right)\right)$. If $\phi$ begins and ends at the same pointed matched circle $\mathcal{Z}$, then it induces an element of the outer automorphism group $\operatorname{Out}(G(\mathcal{Z}), G(\mathcal{Z}))$. That element can be found from the action of $\phi$ on the homology of the surface. In particular, the identity morphism on $F^{\circ}(\mathcal{Z})$ induces the identity outer isomorphism on $G(\mathcal{Z})$.

Remark 4.3 (stabilization) Given $\tau: F^{\circ}\left(\mathcal{Z}_{1}\right) \rightarrow F^{\circ}\left(\mathcal{Z}_{2}\right)$ and its stabilization $\stackrel{\circ}{\tau}$ : $F\left(\stackrel{\circ}{\mathcal{Z}}_{1}\right) \rightarrow F\left(\stackrel{\circ}{\mathcal{Z}}_{2}\right)$, the bimodule $\widehat{\mathcal{D A}}(\tau)$ is again an appropriate restriction of $\widehat{\mathcal{D A}}(\stackrel{\circ}{\tau})$. This follows from the corresponding relations between $\widehat{\mathcal{A A}}(\mathcal{Z})$ and $\widehat{\mathcal{A A}}(\mathcal{Z})$, for any pointed matched circle $\mathcal{Z}$. Taking box tensor products, the stabilization property extends to a relation between $\widehat{\mathcal{D A}}(\phi, \tau)$ and $\widehat{\mathcal{D A}}(\stackrel{\circ}{\phi}, \stackrel{\circ}{\boldsymbol{\tau}})$, where $\stackrel{\circ}{\phi}$ is the element of the mapping class groupoid that acts as identity on the adjoined $\mathcal{Z}^{1}$, and as $\phi$ elsewhere, and where $\stackrel{\circ}{\tau}$ is the extension of the factorization $\boldsymbol{\tau}$.

The corresponding duality statements will be left to the end of Section 4 . By then we will have defined all the other bimodule invariants for surface diffeomorphisms.

\section{B The involution relation}

In this section we will verify the involution relation. Figure 10 shows one of the possible cases: overslide in the upward direction. The computations for overslide in the downward direction, and for underslides over a pair of points at distance greater than 2 from each other, are similar.

Recall that the box tensor product is generated by three types of generators:

$$
{ }_{(*)} X_{(*)} X_{(*)}, \quad{ }_{(1)} X_{(1)} Y_{(2)}, \quad{ }_{(1)} Y_{(2)} X_{(2)} .
$$

For each type $X Y$ generator, there is a corresponding type $Y X$ generator that occupies the same unnumbered pairs. The plan is to cancel out pairs of $X Y$ and $Y X$ generators using this correspondence, and show that the resulting bimodule satisfies the four conditions in Lemma 3.8 .

There are five domains that contribute type $D A$ arrows of interest. They are shown in Figure 11. Domain $C$ contributes an arrow with no $A$-side inputs and idempotent $D$-side output from any $X Y$ generator to the corresponding $Y X$ generator. This allows us to cancel all $X Y$ and $Y X$ generators using the cancellation lemma. We now focus on the resulting bimodule, with the type $X X$ generators. 


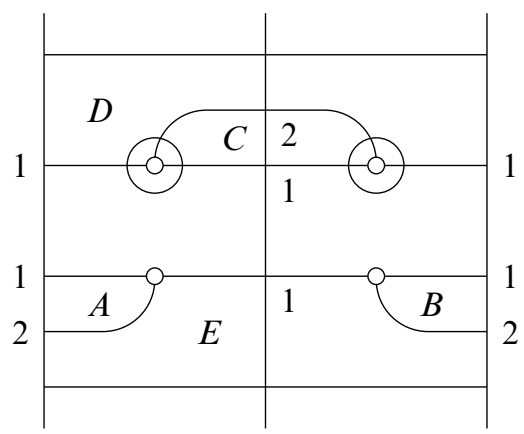

Figure 11: Domains $A-E$ are connected components of $\mathcal{H} \backslash\{\boldsymbol{\alpha}, \boldsymbol{\beta}\}$ containing the respective letters. The domains that contribute the type $D A$ arrows of interest are $A, B, C, D+C$, and $E+C$. If the upper point in pair 1 (resp. the visible point in pair 2) is the topmost (resp. bottommost) point in the pointed matched circle, then the domain $D+C$ (resp. $E+C$ ) does not exist.

This bimodule clearly satisfies (ID-1). Condition (ID-2) can be checked by explicit grading computations, which use only the combinatorial features of the Heegaard diagram. In particular, the fact that the induced map $\phi \in \operatorname{Out}(G(\mathcal{Z}), G(\mathcal{Z}))$ is the identity is equivalent to the fact that the action of this composition of arcslides on $H_{1}(F(\mathcal{Z}))$ is the identity. The stability condition (ID-4) follows from Remark 4.3.

It remains to verify (ID-3). For this, we need to classify all arrows whose coefficients have length at most one on both boundaries. Such an arrow either exists before applying the cancellation lemma, or is produced via a zigzag. In the first case, they correspond to one connected domain between type $X X$ generators. They include trivial horizontal strips in regions away from the slide, the domain $D+C$, and the domain $E+C$. In the second case, the zigzag must be of the form

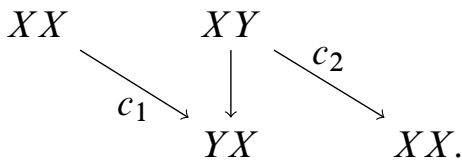

The coefficient $c_{1}$ must have length one on the left boundary and length zero on the right, and $c_{2}$ must have length zero on the left and length one on the right, or viceversa. Looking at the Heegaard diagram, the only possibility is that $c_{1}$ is produced by domain $A$ and $c_{2}$ by domain $B$.

In what follows, we show that for each of the domains $A, B, D+C$, and $E+C$, and any starting and ending generators with matching idempotents, there is exactly one arrow. These arrows, together with the ones coming from simple horizontal strips, cover each length- 1 interval exactly once, which verifies (ID-3). 
Domains $A$ and $B$ are straightforward since they involve only length- 1 coefficients. The next case is the domain $D+C$. If pair 2 is not occupied, the arrow follows from (DA5) and (DA12):
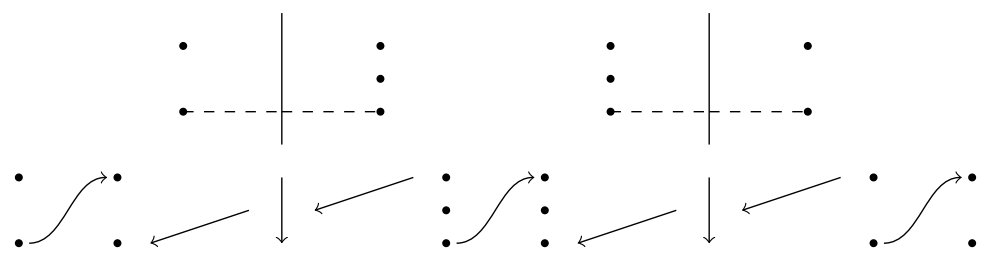

If pair 2 is occupied, then the type $D A$ arrows on the left side depends on the ordering. However, in either case we get the same arrow after box tensoring. If the upper interval comes first, it follows from (DA6) and (DA10):
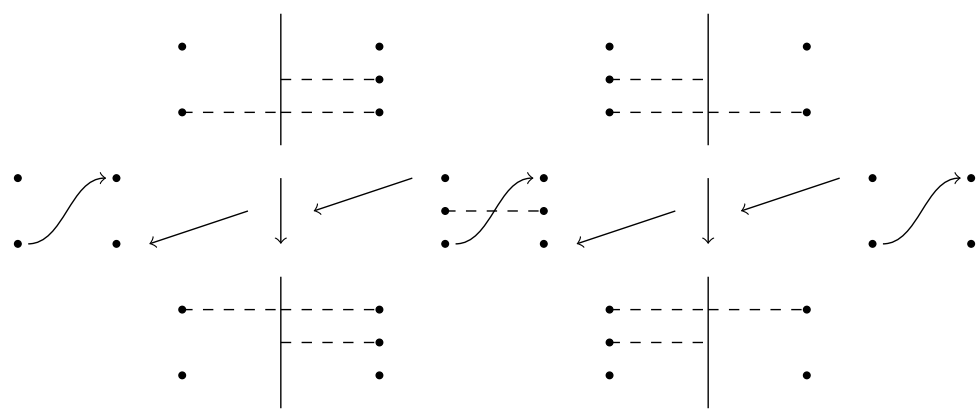

Otherwise, it follows from (DA9), (DA7), and (DA11):

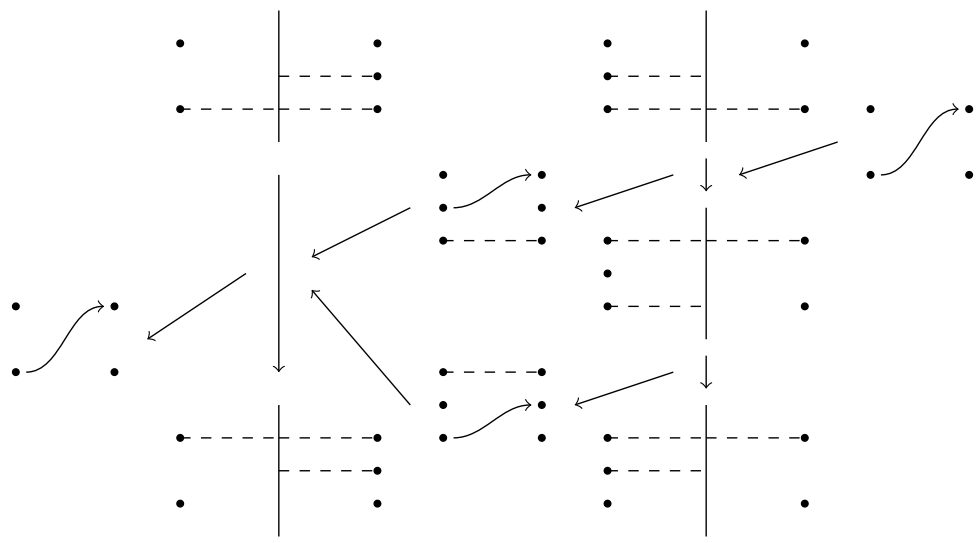


Now for the domain $E+C$. If pair 1 is occupied, the arrow follows from (DA32) and (DA37):

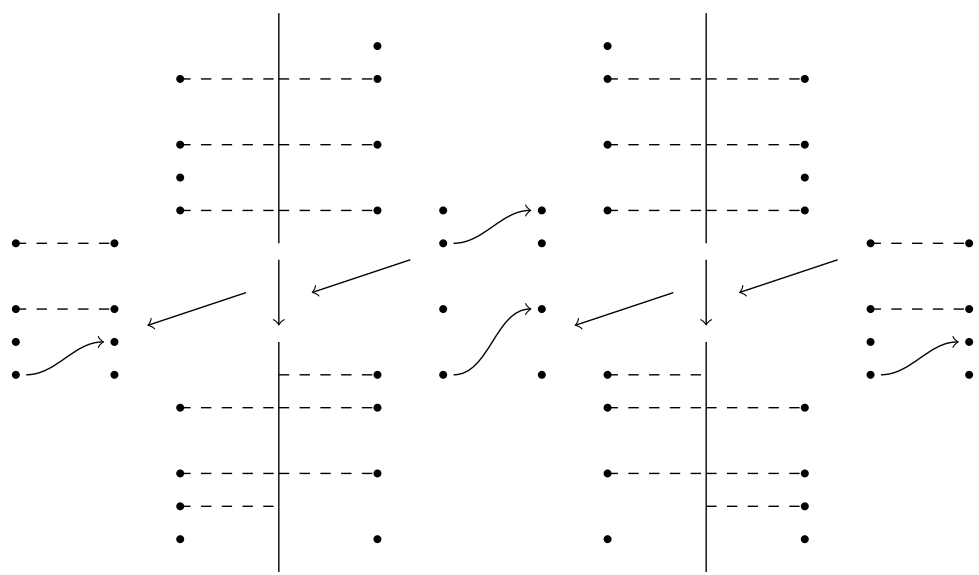

If pair 1 is unoccupied, the arrow follows from (DA31), (DA30), and (DA36):

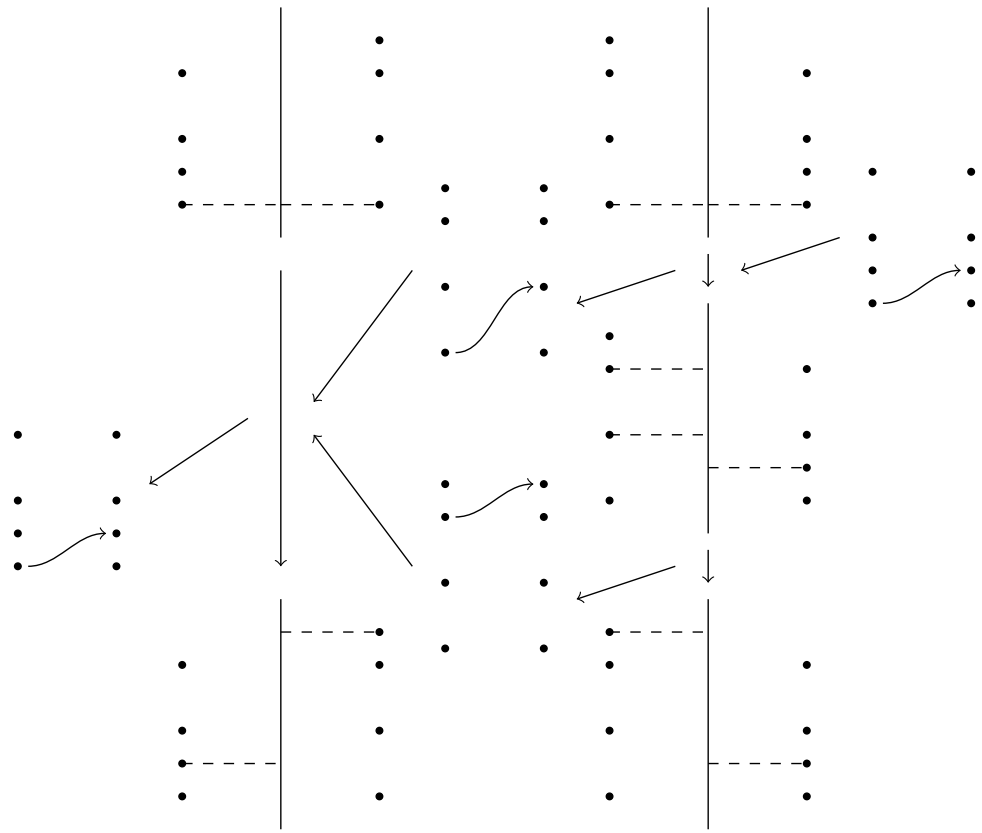

This finishes the verification of the involution relation, except for the case of a short underslide. The computations in that case involve size- 2 intervals where the top and bottom points are paired, so we consider them separately. The diagram is shown in Figure 12. 


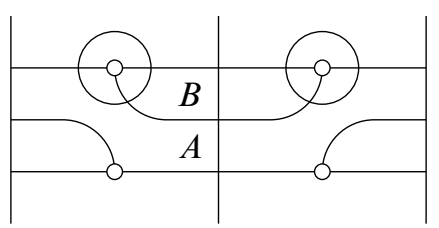

Figure 12: Diagram for involution in the short underslide case. Domains $A$ and $B$ are connected components of $\mathcal{H} \backslash\{\boldsymbol{\alpha}, \boldsymbol{\beta}\}$ containing the respective letters.

The only difference in the verification is computing the arrow covering the upper length- 1 interval. This arrow comes from the domain $A+2 B$, and is produced by (DA22), (DA24), and (DA21):

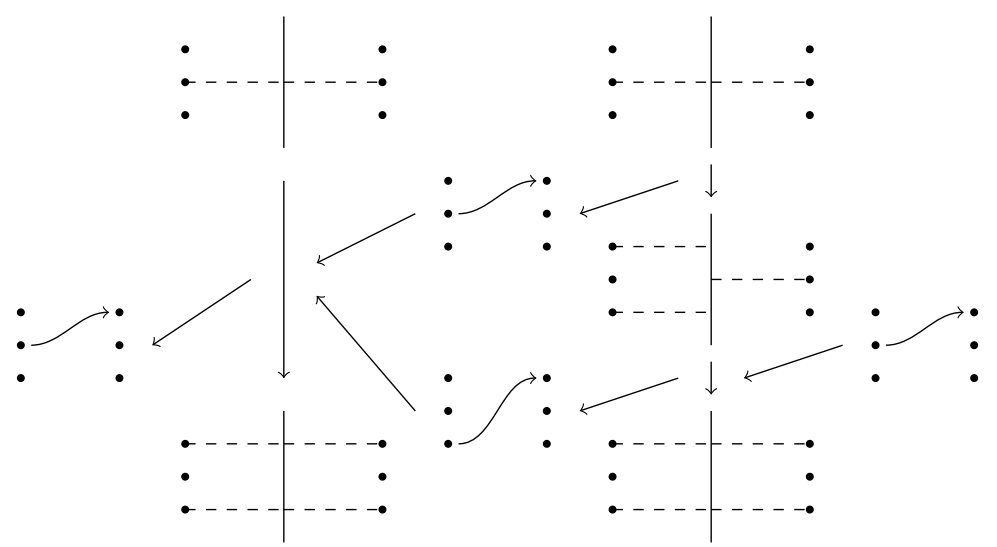

This concludes all cases of the involution relation. Two results follow immediately from this relation.

Corollary 4.4 The bimodule $\widehat{\mathcal{D A}}(\tau)$ is quasi-invertible, and the same is true for any box tensor product of such bimodules.

Proof For a single arcslide, the quasi-inverse is given by $\widehat{\mathcal{D A}}\left(\tau^{-1}\right)$. It is clear that box tensor products of quasi-invertible bimodules are also quasi-invertible.

The computations here allow us to prove a uniqueness statement on $\widehat{\mathcal{D D}}(\tau)$. A similar statement is proven in [11].

Corollary 4.5 Let $\tau: F^{\circ}\left(\mathcal{Z}_{1}\right) \rightarrow F^{\circ}\left(\mathcal{Z}_{2}\right)$ be an arcslide. If a bimodule $\mathcal{A}\left(\mathcal{Z}_{1}\right), \mathcal{A}\left(-\mathcal{Z}_{2}\right) M$ is stable, has the same generators and gradings as $\widehat{\mathcal{D D}}(\tau)$, and its type $D D$ action matches that of $\widehat{\mathcal{D D}}(\tau)$ on all arrows with total lengths of coefficients at most 3 , then $M$ is homotopy equivalent to $\widehat{\mathcal{D D}}(\tau)$. 


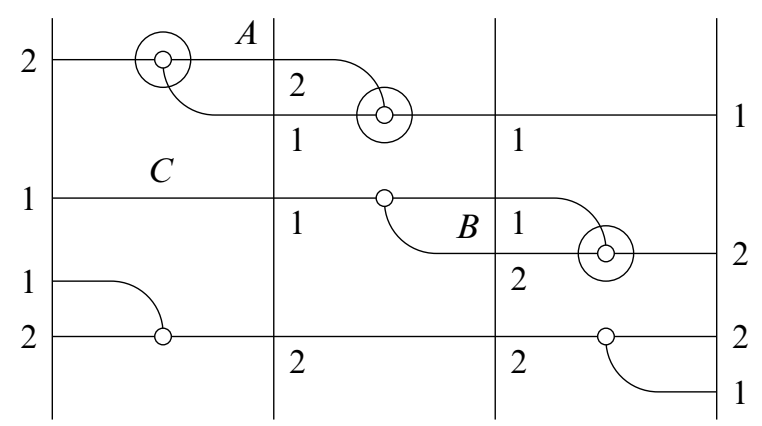

Figure 13: Heegaard diagram for the triangle relation. Domains $A, B$ and $C$ are connected components of $\mathcal{H} \backslash\{\boldsymbol{\alpha}, \boldsymbol{\beta}\}$ containing the respective letters.

Proof Let $M_{D A}=\widehat{\mathcal{A A}}(\mathbb{I}) \otimes M$. Since we only used type $D D$ arrows whose coefficients have total length at most 3 in this section, we can perform the same computations on $M_{D A}$ as on $\widehat{\mathcal{D A}}(\tau)$, showing that

$$
M_{D A} \otimes \widehat{\mathcal{D A}}\left(\tau^{-1}\right) \simeq \mathbb{I} \simeq \widehat{\mathcal{D A}}(\tau) \otimes \widehat{\mathcal{D A}}\left(\tau^{-1}\right) .
$$

Since $\widehat{\mathcal{D A}}\left(\tau^{-1}\right)$ is quasi-invertible, we see $M_{D A}$ is homotopy equivalent to $\widehat{\mathcal{D A}}(\tau)$. Since $\widehat{\mathcal{A A}}(\mathbb{I})$ is also quasi-invertible, we see $M$ is homotopy equivalent to $\widehat{\mathcal{D D}}(\tau)$.

\section{C Other relations on arcslides}

For each of the other relations on arcslides, we check the conditions in Lemma 3.9. Condition (ID-2) is checked by grading computations as before. (ID-3) follows from Corollary 4.4, with the quasi-inverse $M^{\prime}$ being the box tensor product of the inverse arcslides in the opposite order. (ID-4) follows from Remark 4.3. It remains to verify (ID-1); with the same technique given here we can show (ID-1) for the inverse $M^{\prime}$.

4C1 Triangle For the triangle relation, the Heegaard diagram for one of the possible cases is shown in Figure 13. The other cases differ from this one only by switching the ordering of the points and underslides with overslides. The enumeration of generators, and which pairs of generators can be cancelled, are essentially similar.

The roles of the numbered pairs are as follows:

- Arcslide $1 \quad C=2, B=1$.

- Arcslide $2 C=1, B=2$.

- Arcslide $3 C=2, B=1$.

Only the sequence $Y X Y$ is forbidden. For that sequence, pair 1 must be occupied after the first arcslide, and therefore after the second arcslide, so type $Y$ is not possible at the third arcslide. 
The possible types are these:
${ }_{(*)} X_{(*)} X_{(*)} X_{(*)}$,
(2) $Y_{(1)} X_{(1)} X_{(1)}$,
(1) $X_{(1)} Y_{(2)} X_{(2)}$,
(2) $X_{(2)} X_{(2)} Y_{(1)}$,
(2) $Y_{(1)} Y_{(2)} X_{(2)}$,
(1) $X_{(1)} Y_{(2)} Y_{(1)}$,
(2) $Y_{(1)} Y_{(2)} Y_{(1)}$,
(1) $X_{(1)} Y_{(2)} X_{(2)}$.

There are two domains that give rise to cancellable arrows: domain $A$ and $B$ as shown in the figure. Domain $A$ gives rise to arrows from $Y Y *$ to $X X *$, and domain $B$ gives rise to arrows from $* Y Y$ to $* X X$. So the cancellable arrows are these:

$$
\begin{gathered}
Y Y Y \rightarrow X X Y, \quad Y Y Y \rightarrow Y X X \\
{\text { (1) } X Y Y_{(1)}} \rightarrow_{(1)} X X X_{(1)}, \quad{ }_{(2)} Y Y X_{(2)} \rightarrow_{(2)} X X X_{(2)} .
\end{gathered}
$$

We choose to cancel everything except the second set $Y Y Y \rightarrow Y X X$ (cancelling the first set of arrows eliminates the option of cancelling the second). These are the remaining generators:
(2) $Y X X_{(1)}$,
(1) $X Y X_{(2)}$,
() $X X X_{()}$,
(12) $X X X_{(12)}$

Since each type of idempotents at the two ends occurs exactly once, we have verified (ID-1). Note that pair 1 at the left becomes pair 2 at the right, and vice versa, under the bijection of pairs coming from the equality of pointed matched circles at the two ends. For the triangle relation, it is not immediately clear that there exists a refined relative grading where all generators have grading zero, so we give more details on verifying this condition. Choose a generator in class ${ }_{(2)} Y X X_{(1)}$ as the base generator (with refined grading zero). To verify that any generator of class (1) $X Y X_{(2)}$ has grading zero, it suffices to check that any potential domain connecting them has the expected grading. The domain $B+C$ is such a domain. Its grading can be computed to be the same as that of a simple horizontal strip in the Heegaard diagram for identity, with the same boundaries at the two sides. If the genus is greater than 2, then generators of type ${ }_{(} X X X_{()}$and ${ }_{(12)} X X X_{(12)}$ exist. They are connected to (2) $Y X X_{(1)}$ or (1) $X Y X_{(2)}$ by horizontal strips above either $A$ or $B$. These domains also have the same gradings as the simple horizontal strips in the diagram for identity with the same boundaries, so the latter two types of generators must also have grading zero.

4C2 Commutativity The Heegaard diagram for one of the cases of the commutativity relation is shown in Figure 14; as in the triangle case, the other possibilities are similar. The role of the numbered pairs are as follows:

- Arcslide $1 \quad C=1, B=2$.

- Arcslide $2 C=3, B=4$.

- Arcslide $3 C=1, B=2$.

- Arcslide $4 C=3, B=4$. 


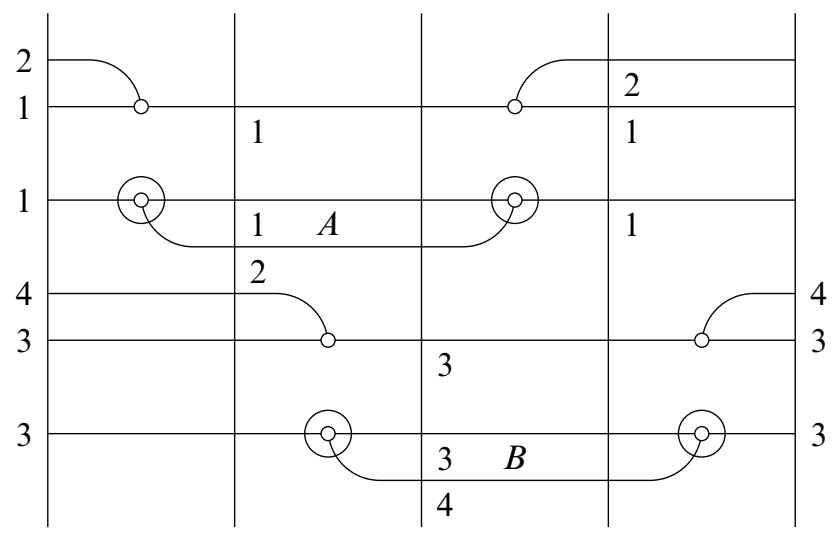

Figure 14: Heegaard diagram for the commutativity relation. Domains $A$ and $B$ are connected components of $\mathcal{H} \backslash\{\boldsymbol{\alpha}, \boldsymbol{\beta}\}$ containing the respective letters.

The restriction on the types is that at most one of the types at arcslides 1 and 3 can be $Y$, and at most one at arcslides 2 and 4 can be $Y$. The possibilities are:
${ }_{(*)} X_{(*)} X_{(*)} X_{(*)} X_{(*)}$,
${ }_{(1)} Y_{(2)} X_{(2)} X_{(2)} X_{(2)}(3$ or 4$)$,
(1) $X_{(1)} X_{(1)} Y_{(2)} X_{(2)}(3$ or 4$)$,
(3) $X_{(3)} Y_{(4)} X_{(4)} X_{(4)}(1$ or 2$)$,
(3) $X_{(3)} X_{(3)} X_{(3)} Y_{(4)}(1$ or 2$)$,
(13) $Y_{(23)} Y_{(24)} X_{(24)} X_{(24)}$,
(13) $X_{(13)} Y_{(14)} Y_{(24)} X_{(24)}$,
(13) $X_{(13)} X_{(13)} Y_{(23)} Y_{(24)}$,

(13) $Y_{(23)} X_{(23)} X_{(23)} Y_{(24)}$,

where ( 3 or 4 ) means "with pairs 3 and/or 4 possibly added to each idempotent".

The two domains giving rise to cancellable arrows are labelled $A$ and $B$ in the Figure 14 . Domain $A$ gives rise to arrows from $Y a X b$ to $X a Y b$, for any valid choice of $a, b \in$ $\{X, Y\}$. Likewise, domain $B$ gives rise to arrows from $a Y b X$ to $a X b Y$. So the cancellable arrows are:

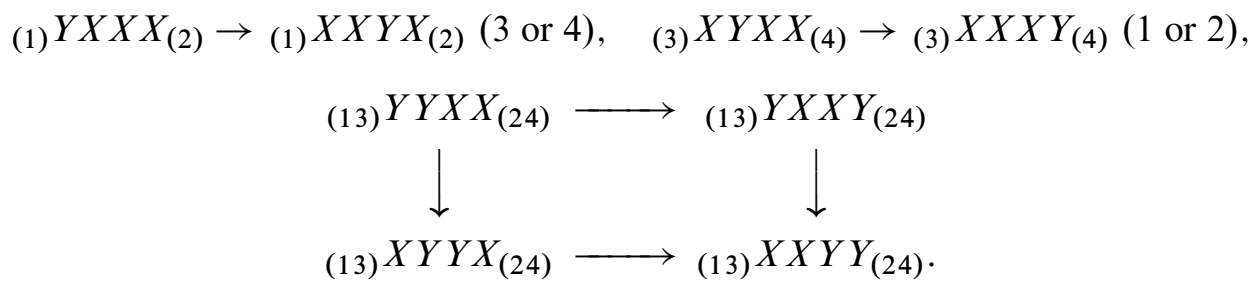

The first two arrows cancel all generators with one $Y$. For generators with two $Y$ 's, we can either cancel both horizontal arrows or both vertical arrows in the square above. In the end, only generators of type ${ }_{(*)} X X X X_{(*)}$ remain, which verifies (ID-1). 


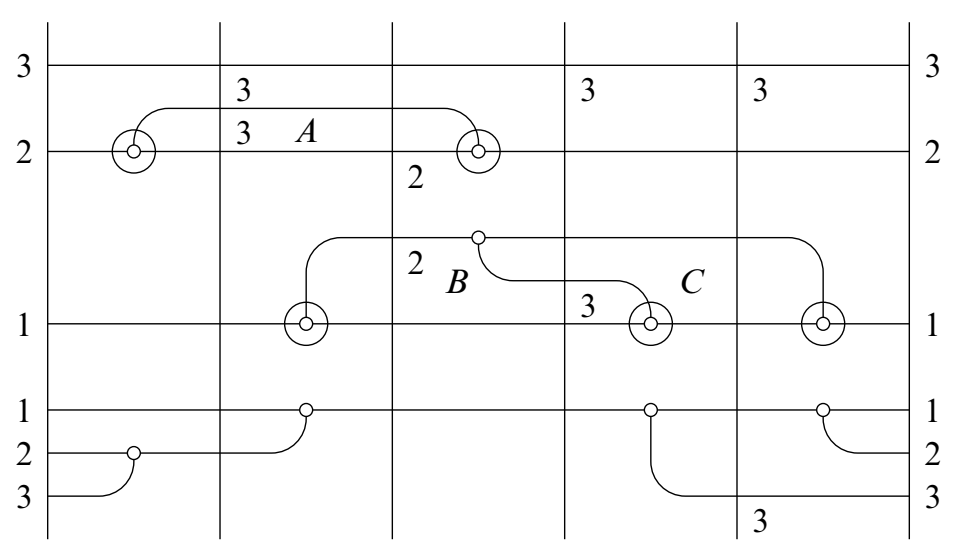

Figure 15: Heegaard diagram for the pentagon relation. Domains $A, B$, and $C$ are connected components of $\mathcal{H} \backslash\{\boldsymbol{\alpha}, \boldsymbol{\beta}\}$ containing the respective letters.

4C3 Left and right pentagon The Heegaard diagram for one of the cases of the left pentagon relation is shown in Figure 15. Other cases of the left and right pentagon relation are similar.

The role of the numbered pairs are as follows:

- Arcslide $1 C=2, B=3$.

- Arcslide $2 C=1, B=2$.

- Arcslide $3 C=2, B=3$.

- Arcslide $4 C=1, B=3$.

- Arcslide $5 C=1, B=2$.

The possible types are:

- ${ }_{(*)} X_{(*)} X_{(*)} X_{(*)} X_{(*)} X_{(*)}$,

- (12) $Y_{(13)} Y_{(23)} X_{(23)} X_{(23)} X_{(23)}$,

- (12) $X_{(12)} X_{(12)} Y_{(13)} X_{(13)} Y_{(23)}$,

- ${ }_{(12)} Y_{(13)} X_{(13)} X_{(13)} X_{(13)} Y_{(23)}$,

- (1) $X_{(1)} Y_{(2)} Y_{(3)} X_{(3)} X_{(3)}$,

- ${ }_{(2)} Y_{(3)} X_{(3)} X_{(3)} X_{(3)} X_{(3)}$, (12) $Y_{(13)} X_{(13)} X_{(13)} X_{(13)} X_{(13)}$,

- ${ }_{(1)} X_{(1)} Y_{(2)} X_{(2)} X_{(2)} X_{(2)}$, (13) $X_{(13)} Y_{(23)} X_{(23)} X_{(23)} X_{(23)}$,

- ${ }_{(2)} X_{(2)} X_{(2)} Y_{(3)} X_{(3)} X_{(3)}$, (12) $X_{(12)} X_{(12)} Y_{(13)} X_{(13)} X_{(13)}$,

- ${ }_{(1)} X_{(1)} X_{(1)} X_{(1)} Y_{(3)} X_{(3)}$, (12) $X_{(12)} X_{(12)} X_{(12)} Y_{(23)} X_{(23)}$,

- ${ }_{(1)} X_{(1)} X_{(1)} X_{(1)} X_{(1)} Y_{(2)}$, (13) $X_{(13)} X_{(13)} X_{(13)} X_{(13)} Y_{(23)}$. 


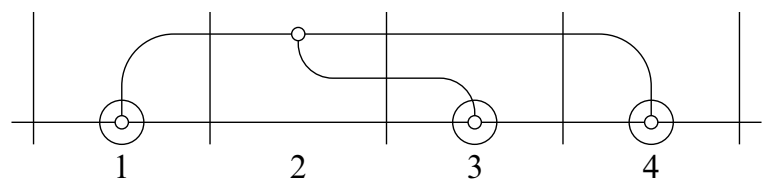

Figure 16: Domain $B+C$

Domains $A, B$, and $C$, respectively, give rise to the following arrows:

$$
X X Y * * \rightarrow Y X X * *, \quad * X X Y * \rightarrow * Y Y X *, \quad * * Y X Y \rightarrow * * X Y X .
$$

Other domains that may give arrows are $B+C$ and $A+B$. We first analyze $B+C$, showing that it will always contribute an arrow whenever idempotent matches. The calculation involves box tensoring the four type $D A$ bimodules as shown in Figure 16.

The arrow needed in the fourth piece is simple. For the third, there are several ways to cover the domain. First, if pair 3 is unoccupied in the middle pieces, use (DA5):

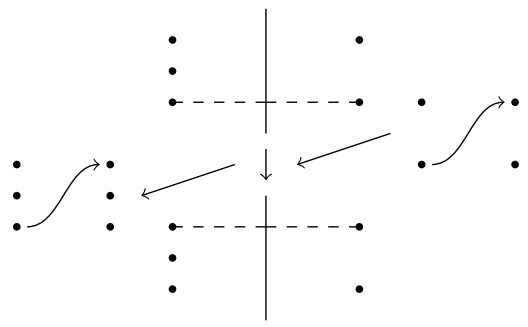

If pair 3 is occupied, there are two different ways: (DA6), or (DA9) and (DA7):

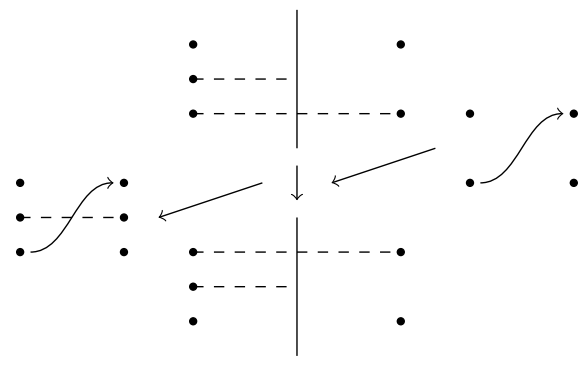

and

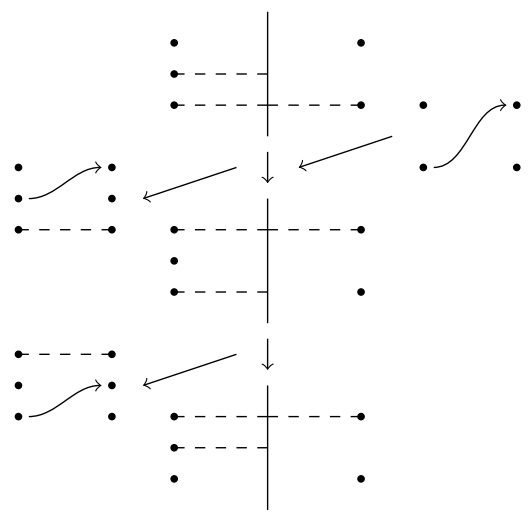

Now looking at the possible arrows in the second piece, we see there is always exactly one way to continue forming the arrow in the box tensor product to the second piece (and then trivially to the first piece). If pair 3 is unoccupied, we use (DA12). If pair 3 is occupied, we use either (DA10) or (DA11), depending on the ordering $<_{\mathcal{Z}}$. This 
shows that the domain $B+C$ gives rise to the following arrows:

$$
\begin{aligned}
*_{(1)} X_{(1)} X_{(1)} X_{(1)} Y_{(2)} & \rightarrow *_{(1)} Y_{(2)} X_{(2)} X_{(2)} X_{(2)}, \\
*_{(13)} X_{(13)} X_{(13)} X_{(13)} Y_{(23)} & \rightarrow *{ }_{(13)} Y_{(23)} X_{(23)} X_{(23)} X_{(23)}
\end{aligned}
$$

Finally, we consider the domain $A+B$. This domain potentially contributes arrows of the form $X X X X * \rightarrow Y Y X X *$. The only possible choice of idempotents is

$$
{ }_{(12)} X_{(12)} X_{(12)} X_{(12)} Y_{(23)} X_{(23)} \rightarrow{ }_{(12)} Y_{(13)} Y_{(23)} X_{(23)} X_{(23)} X_{(23)}
$$

Rather than computing the type $D A$ arrows for this domain like in the previous case, we note that the sequence

$$
{ }_{(12)} X X Y X Y_{(23)} \rightarrow{ }_{(12)} Y X X X Y_{(23)} \rightarrow{ }_{(12)} Y Y X X X X_{(23)}
$$

must cancel against something in the type $D A$ structure equation. This is possible only if the domain $A+B$ contributes an arrow.

In summary, the cancellable arrows are these:

$$
\begin{aligned}
& \text { (1) } X X X X Y_{(2)} \rightarrow \text { (1) } X Y X X X_{(2)}, \quad \text { (13) } X X X X Y_{(23)} \rightarrow \text { (13) } X Y X X X_{(23)} \text {, } \\
& \text { (2) } X X Y X X_{(3)} \rightarrow{ }_{(2)} Y X X X X_{(3)}, \quad \text { (12) } X X Y X X_{(13)} \rightarrow{ }_{(12)} Y X X X X X_{(13)} \text {, } \\
& \text { (1) } X X X Y X_{(3)} \rightarrow{ }_{(1)} X Y Y X X_{(3)} \text {, }
\end{aligned}
$$$$
\text { (12) } X X Y X Y_{(23)}
$$

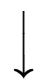

(12) $X X X Y X_{(23)}$
(12) $Y X X X Y_{(23)}$

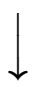

(12) $Y Y X X X_{(23)}$

The four types of generators starting with (12) and ending with (23) form the square above, and are cancelled using either the horizontal or vertical arrows. The other ten types of generators containing at least one $Y$ are cancelled using the first five arrows. So only generators of type ${ }_{(*)} X X X X X_{(*)}$ remain, which verifies (ID-1).

This concludes the proof of Theorem 2.2, showing that the bimodule $\widehat{\mathcal{D A}}(\phi, \tau)$ is independent of the choice of factorization $\tau$ up to homotopy equivalence. This allows us to write $\widehat{\mathrm{CFDA}}(\phi)$ for the homotopy equivalence class of $\widehat{\mathcal{D A}}(\phi, \tau)$, and define the other invariants $\widehat{\mathrm{CFDD}}(\phi), \widehat{\mathrm{CFA} A}(\phi)$, and $\widehat{\mathrm{CFAD}}(\phi)$ combinatorially by box tensoring with appropriate identity bimodules.

We finish with a discussion of how duality on $\widehat{\mathcal{D D}}(\tau)$ extends to the other bimodule invariants. 
Lemma 4.6 For any element $\phi: F^{\circ}\left(\mathcal{Z}_{1}\right) \rightarrow F^{\circ}\left(\mathcal{Z}_{2}\right)$ of the strongly based mapping class groupoid, we have

$$
\mathcal{A}\left(-\mathcal{Z}_{1}\right) \widehat{\mathrm{CFAD}}\left(\phi^{-1}\right)_{\mathcal{A}\left(-\mathcal{Z}_{2}\right)} \simeq \overline{\mathcal{A}\left(\mathcal{Z}_{1}\right) \mathrm{C} \widehat{\mathrm{FDA}}(\phi)_{\mathcal{A}\left(\mathcal{Z}_{2}\right)}} .
$$

Proof First, we consider the case of an arcslide $\tau$. Using the definition of $\widehat{\mathrm{CFAD}}$ and the fact that $\widehat{\mathrm{CFA}} \mathrm{A}(\mathbb{I})$ and $\widehat{\mathrm{CFDD}}(\mathbb{I})$ are quasi-inverses, we have

$$
\mathcal{A}\left(\mathcal{Z}_{2}\right), \mathcal{A}\left(-\mathcal{Z}_{1}\right) \widehat{\operatorname{CFDD}}\left(\tau^{-1}\right) \simeq \mathcal{A}\left(-\mathcal{Z}_{1}\right) \widehat{\operatorname{CFAD}}\left(\tau^{-1}\right)_{\mathcal{A}\left(-\mathcal{Z}_{2}\right)} \otimes^{\mathcal{A}\left(\mathcal{Z}_{2}\right), \mathcal{A}\left(-\mathcal{Z}_{2}\right)} \widehat{\mathrm{CFDD}}\left(\mathbb{I}_{\mathcal{Z}_{2}}\right) .
$$

On the other hand,

$$
\begin{aligned}
& \mathcal{A}\left(-\mathcal{Z}_{1}\right), \mathcal{A}\left(\mathcal{Z}_{2}\right) \widehat{\widehat{\mathrm{FDD}}(\tau)} \simeq \overline{\mathcal{A}\left(\mathcal{Z}_{1}\right) \mathrm{C} \widehat{\mathrm{FD} A}(\tau)_{\mathcal{A}\left(\mathcal{Z}_{2}\right)} \otimes \mathcal{A}\left(\mathcal{Z}_{2}\right), \mathcal{A}\left(-\mathcal{Z}_{2}\right) \widehat{\mathrm{CFDD}}\left(\mathbb{I}_{\mathcal{Z}_{2}}\right)} \\
& \left.\simeq \mathcal{A}\left(-\mathcal{Z}_{1}\right) \widehat{\widehat{\operatorname{CFDA}}(\tau)}\right)_{\mathcal{A}\left(-\mathcal{Z}_{2}\right)} \otimes^{\mathcal{A}\left(-\mathcal{Z}_{2}\right), \mathcal{A}\left(\mathcal{Z}_{2}\right)} \overline{\widehat{\operatorname{CDDD}}\left(\mathbb{I}_{\mathcal{Z}_{2}}\right)} .
\end{aligned}
$$

By the remarks on duality at the end of Section $2 \mathrm{E}$, we see $\widehat{\mathrm{CFDD}}\left(\tau^{-1}\right)$ and $\widehat{\widehat{\mathrm{CDDD}}(\tau)}$ are homotopy equivalent after switching the two algebra actions. It is also clear from the construction of $\widehat{\mathrm{CFDD}}\left(\mathbb{I}_{\mathcal{Z}_{2}}\right)$ that it is isomorphic to $\widehat{\mathrm{CFDD}}\left(\mathbb{I}_{\mathcal{Z}_{2}}\right)$ after switching the algebra actions. This implies (25) for arcslides $\tau$.

For a general surface diffeomorphism $\phi$, factor it into arcslides $\tau_{i}$. The statement then follows from the case of arcslides, and the fact that taking duals distributes over the box tensor product.

\section{The 3-manifold invariant}

In this section, we prove Theorem 2.4, showing that the homotopy type of the chain complex $\widehat{\mathrm{HF}}$ given in Construction 2.3 does not depend on the choices made. There are two main components of the proof, given by the two lemmas below.

Let $\mathrm{MCG}_{0}\left(\mathcal{Z}^{g}\right)$ denote the strongly based mapping class group on $F_{g, 1}$, parametrized by the genus $g$ split pointed matched circle $\mathcal{Z}^{g}$. Recall that $\boldsymbol{H}^{g}$ denotes the 0 -framed handlebody, and its orientation reversal $-\boldsymbol{H}^{g}$ is the $\boldsymbol{\infty}$-framed handlebody.

Lemma 5.1 (stabilization) Let $\psi$ be an element of $\mathrm{MCG}_{0}\left(\mathcal{Z}^{g}\right)$. Consider $F_{g+1,1}$, parametrized by $\mathcal{Z}^{g+1}$ as the surface obtained from $F_{g, 1}$ by adding a handle in a neighborhood of the basepoint. Let $\dot{\psi}$ be the element of $\mathrm{MCG}_{0}\left(\mathcal{Z}^{g+1}\right)$ that fixes the new handle and acts as $\psi$ elsewhere. Then

$$
\begin{aligned}
\left(\widehat{\mathrm{CFA} A}(\psi) \otimes \widehat{\mathrm{CFD}}\left(\boldsymbol{H}^{g}\right)\right) & \otimes \widehat{\mathrm{CFD}}\left(-\boldsymbol{H}^{g}\right) \\
& \simeq\left(\widehat{\mathrm{CFA} A}(\stackrel{\circ}{\psi}) \otimes \widehat{\mathrm{CFD}}\left(\boldsymbol{H}^{g+1}\right)\right) \otimes \widehat{\mathrm{CFD}}\left(-\boldsymbol{H}^{g+1}\right) .
\end{aligned}
$$


Definition 5.2 Define $\mathrm{MCG}_{0}^{\beta}\left(\mathcal{Z}^{g}\right)$ to be the subgroup of $\mathrm{MCG}_{0}\left(\mathcal{Z}^{g}\right)$ consisting of maps that extend to automorphisms of $\boldsymbol{H}^{g}$. Likewise, define $\operatorname{MCG}_{0}^{\alpha}\left(\mathcal{Z}^{g}\right)$ to be the subgroup of $\mathrm{MCG}_{0}\left(\mathcal{Z}^{g}\right)$ consisting of maps that extend to automorphisms of $-\boldsymbol{H}^{g}$ (using identification $\mathcal{Z}^{g}=-\mathcal{Z}^{g}$ to consider $-\boldsymbol{H}^{g}$ as parametrized by $\mathcal{Z}^{g}$ ).

Lemma 5.3 (reparametrization of the 0 -framed handlebody) For each element $\phi \in$ $\operatorname{MCG}_{0}^{\beta}\left(\mathcal{Z}^{g}\right)$, we have

$$
\mathcal{A}\left(-\mathcal{Z}^{g}\right) \widehat{\mathrm{CFAD}}(\phi)_{\mathcal{A}\left(-\mathcal{Z}^{g}\right)} \otimes^{\mathcal{A}\left(-\mathcal{Z}^{g}\right)} \widehat{\mathrm{CFD}}\left(\boldsymbol{H}^{g}\right) \simeq \mathcal{A ( - \mathcal { Z } ^ { g } )} \widehat{\mathrm{CFD}}\left(\boldsymbol{H}^{g}\right) .
$$

We first show that these two lemmas imply Theorem 2.4.

Proof of Theorem 2.4 There are two choices made in Construction 2.3: the choice of Heegaard splitting $Y=Y_{1} \cup Y_{2}$, and choice of parametrizations of $Y_{1}$ and $Y_{2}$ by standard handlebodies. It is well known that any two Heegaard splittings become isotopic after a finite number of stabilizations. Also, any stabilization can be isotopied to the standard one, adding a handle in a neighborhood of the basepoint. If $\psi$ is a valid choice of element in $\mathrm{MCG}_{0}\left(\mathcal{Z}^{g}\right)$ in the second stage of the construction, then $\stackrel{\circ}{\psi}$ is a valid choice of element in $\mathrm{MCG}_{0}\left(\mathcal{Z}^{g+1}\right)$ after a standard stabilization. So Lemma 5.1 implies that Construction 2.3 is invariant under stabilizations.

Now we consider choice of parametrizations of $Y_{1}$ and $Y_{2}$. Recall $\psi=\bar{f}_{2 *}^{-1} \circ u \circ f_{1 *}$, where $u: \partial Y_{1} \rightarrow-\partial Y_{2}$ is the gluing map, $f_{1}: \boldsymbol{H}^{g} \rightarrow Y_{1}$ is the parametrization of $Y_{1}$ by $\boldsymbol{H}^{g}$, and $f_{2}:-\boldsymbol{H}^{g} \rightarrow Y_{2}$ is the parametrization of $Y_{2}$ by $-\boldsymbol{H}^{g}$. Hence, changing parametrization of $Y_{1}$ changes $\psi$ to $\psi^{\prime}=\psi \circ \phi_{1}$, where $\phi_{1} \in \operatorname{MCG}_{0}^{\beta}\left(\mathcal{Z}^{g}\right)$, and changing parametrization of $Y_{2}$ changes $\psi$ to $\psi^{\prime}=\bar{\phi}_{2}^{-1} \circ \psi$, where $\phi_{2} \in \operatorname{MCG}_{0}^{\alpha}\left(\mathcal{Z}^{g}\right)$.

It remains to show the following:

$$
\begin{aligned}
\widehat{\mathrm{CFA} A}\left(\psi \circ \phi_{1}\right) \otimes \widehat{\mathrm{CFD}}\left(\boldsymbol{H}^{g}\right) \simeq \widehat{\mathrm{CFA} A}(\psi) \otimes \widehat{\mathrm{CFD}}\left(\boldsymbol{H}^{g}\right), \\
\widehat{\mathrm{CFA}}\left(\bar{\phi}_{2}^{-1} \circ \psi\right) \otimes \widehat{\mathrm{CFD}}\left(-\boldsymbol{H}^{g}\right) \simeq \widehat{\mathrm{CFA} A}(\psi) \otimes \widehat{\operatorname{CFD}}\left(-\boldsymbol{H}^{g}\right),
\end{aligned}
$$

for $\phi_{1} \in \operatorname{MCG}_{0}^{\beta}\left(\mathcal{Z}^{g}\right)$ and $\phi_{2} \in \operatorname{MCG}_{0}^{\alpha}\left(\mathcal{Z}^{g}\right)$.

The first equation follows directly from Lemma 5.3:

$$
\begin{aligned}
\widehat{\mathrm{CFAA}}\left(\psi \circ \phi_{1}\right) \otimes \widehat{\mathrm{CFD}}\left(\boldsymbol{H}^{g}\right) & \simeq \widehat{\mathrm{CFA} A}(\psi) \otimes \widehat{\mathrm{CFAD}}\left(\phi_{1}\right) \otimes \widehat{\mathrm{CFD}}\left(\boldsymbol{H}^{g}\right) \\
& \simeq \widehat{\mathrm{CFA} A}(\psi) \otimes \widehat{\mathrm{CFD}}\left(\boldsymbol{H}^{g}\right) .
\end{aligned}
$$

For the second equation, by taking the dual of (27), and using Lemma 4.6, we get

$$
\mathcal{A}\left(\mathcal{Z}^{g}\right) \widehat{\mathrm{CFDA}}\left(\phi^{-1}\right)_{\mathcal{A}\left(\mathcal{Z}^{g}\right)} \otimes^{\mathcal{A}\left(\mathcal{Z}^{g}\right)} \widehat{\mathrm{CFD}}\left(-\boldsymbol{H}^{g}\right) \simeq \mathcal{A}\left(\mathcal{Z}^{g}\right) \widehat{\mathrm{CFD}}\left(-\boldsymbol{H}^{g}\right)
$$




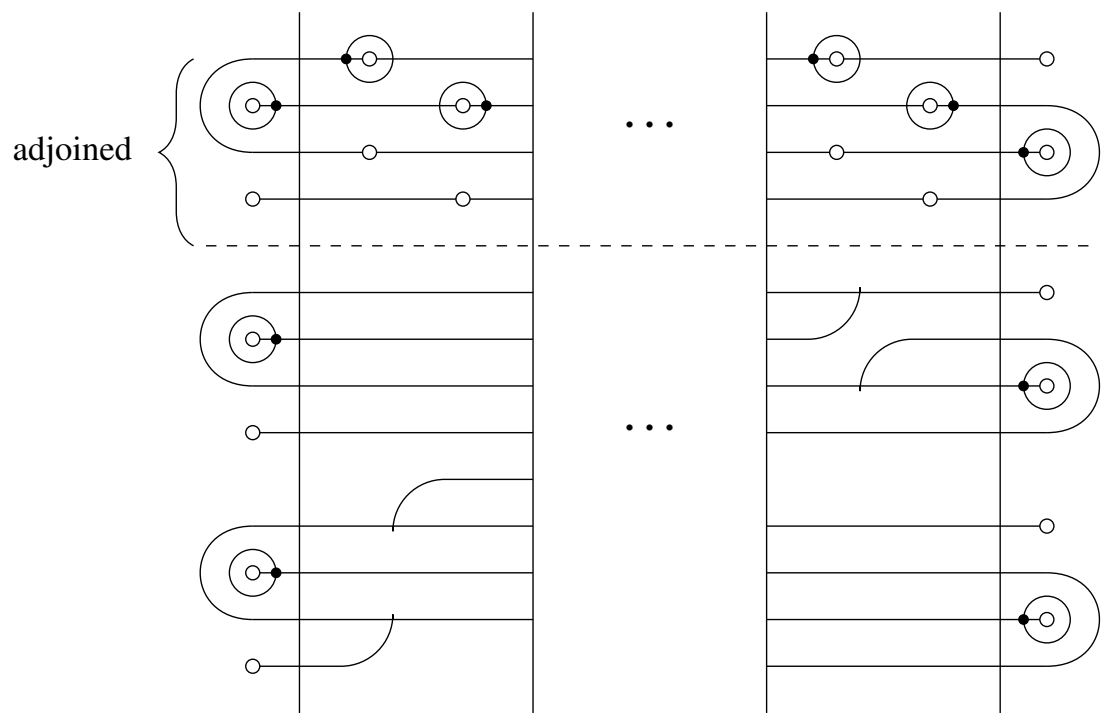

Figure 17: Proof of invariance under stabilization

for any $\phi \in \operatorname{MCG}_{0}^{\beta}\left(\mathcal{Z}^{g}\right)$. Then the second equation follows, as

$$
\begin{aligned}
\widehat{\mathrm{CFAA}}\left(\bar{\phi}_{2}^{-1} \circ \psi\right) \otimes \widehat{\mathrm{CFD}}\left(-\boldsymbol{H}^{g}\right) & \simeq \widehat{\mathrm{CFA} A}(\psi) \otimes \widehat{\mathrm{CFDA}}\left(\bar{\phi}_{2}^{-1}\right) \otimes \widehat{\mathrm{CFD}}\left(-\boldsymbol{H}^{g}\right) \\
& \simeq \widehat{\mathrm{CFA} A}(\psi) \otimes \widehat{\mathrm{CFD}}\left(-\boldsymbol{H}^{g}\right)
\end{aligned}
$$

since $\phi_{2} \in \operatorname{MCG}_{0}^{\alpha}\left(\mathcal{Z}^{g}\right)$ implies $\bar{\phi}_{2} \in \operatorname{MCG}_{0}^{\beta}\left(\mathcal{Z}^{g}\right)$.

Proof of Lemma 5.1 Choose factorization $\boldsymbol{\tau}$ for $\psi$, then $\stackrel{\circ}{\boldsymbol{\tau}}$ is a factorization for $\stackrel{\circ}{\psi}$.

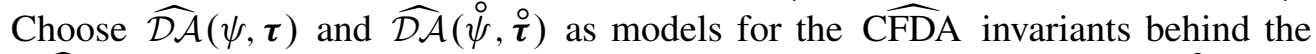
$\widehat{\mathrm{CFA} A}$ invariants. The lemma then follows from the stabilization property for $\widehat{\mathcal{D A}}(\psi, \tau)$. We can see this by comparing the Heegaard diagrams underlying the two sides of (26). First, the Heegaard diagram for $\widehat{\mathcal{D A}}(\stackrel{\circ}{\psi}, \stackrel{\circ}{\boldsymbol{\tau}})$ is constructed from that for $\widehat{\mathcal{D A}}(\psi, \boldsymbol{\tau})$ by adjoining a horizontal strip of diagrams for the identity diffeomorphism of the genus 1 surface at the top. Likewise, the Heegaard diagrams of $\boldsymbol{H}^{g+1}$ and $-\boldsymbol{H}^{g+1}$ are obtained from that of $\boldsymbol{H}^{g}$ and $-\boldsymbol{H}^{g}$ by adjoining diagrams of $\boldsymbol{H}^{1}$ and $-\boldsymbol{H}^{1}$ to the top. These constructions are combined in Figure 17.

By Remark 4.1, generators in the chain complex

$$
\left(\widehat{\mathrm{CFA} A}(\psi) \otimes \widehat{\mathrm{CFD}}\left(\boldsymbol{H}^{g}\right)\right) \otimes \widehat{\mathrm{CFD}}\left(-\boldsymbol{H}^{g}\right)
$$


correspond to certain tuples of intersection points in the part of the diagram below the dashed line in Figure 17, while generators in the chain complex

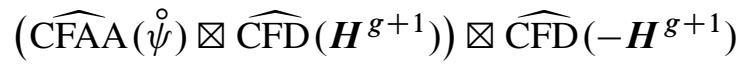

correspond to certain tuples of intersection points in the full diagram. Likewise, there is a correspondence between arrows in the type $D A$ action on the two sides, and domains in appropriate parts of the diagram.

The choice of intersection points in the adjoined portion of the diagram is forced (as marked in the figure), which means that it is the same for all generators in (30). So there is a one-to-one correspondence between generators in (29) and (30). Moreover, since there are no closed domains above the dashed line, all arrows in (30) automatically have domains restricted below the dashed line. By Remark 4.3, there is a one-to-one correspondence between these arrows and the arrows in (29). This shows the chain complexes (29) and (30) are isomorphic, proving Lemma 5.1.

For Lemma 5.3, we need to show

$$
\widehat{\mathrm{CFAD}}(\phi) \otimes \widehat{\mathrm{CFD}}\left(\boldsymbol{H}^{g}\right) \simeq \widehat{\mathrm{CFD}}\left(\boldsymbol{H}^{g}\right)
$$

for any $\phi \in \mathrm{MCG}_{0}^{\beta}\left(\mathcal{Z}_{g}\right)$. It suffices to verify the equation for a set of generators of $\operatorname{MCG}_{0}^{\beta}\left(\mathcal{Z}_{g}\right)$.

We find generators for the strongly based mapping class group by appealing to results on the usual mapping class group. Let $F_{g}$ be the genus $g$ surface with a basepoint. Let $\operatorname{MCG}\left(F_{g}\right)$ be the group of isotopy classes of diffeomorphisms on $F_{g}$ that fixes the basepoint, with isotopies also required to fix the basepoint. It is related to $\operatorname{MCG}_{0}\left(\mathcal{Z}_{g}\right)$ by a short exact sequence (see [4, Section 4.2.5]):

$$
0 \rightarrow \mathbb{Z} \stackrel{\tau_{\partial}}{\longrightarrow} \operatorname{MCG}_{0}\left(\mathcal{Z}_{g}\right) \rightarrow \operatorname{MCG}\left(F_{g}\right) \rightarrow 0
$$

Here $\tau_{\partial}$ maps the generator of $\mathbb{Z}$ to the boundary Dehn twist in $\operatorname{MCG}_{0}\left(\mathcal{Z}_{g}\right)$. This is the element that performs a Dehn twist along a loop parallel to the boundary of $F_{g, 1}$.

There is, likewise, a short exact sequence

$$
0 \rightarrow \mathbb{Z} \stackrel{\tau_{\partial}}{\longrightarrow} \operatorname{MCG}_{0}^{\beta}\left(\mathcal{Z}_{g}\right) \rightarrow \operatorname{MCG}^{\beta}\left(F_{g}\right) \rightarrow 0
$$

where $\operatorname{MCG}^{\beta}\left(F_{g}\right)$ is the subgroup of $\operatorname{MCG}\left(F_{g}\right)$ consisting of restrictions of automorphisms of the 0 -framed handlebody $\boldsymbol{H}^{g}$. This exact sequence shows that a generating set of $\mathrm{MCG}_{0}^{\beta}\left(\mathcal{Z}_{g}\right)$ can be obtained by adding the boundary Dehn twist to the lifting of a generating set of $\mathrm{MCG}^{\beta}\left(F_{g}\right)$. 
A generating set of $\mathrm{MCG}^{\beta}\left(F_{g}\right)$ is given in [20] (the corresponding notation in that paper is $\left.\mathrm{MCG}^{*}\left(F_{g}\right)\right)$. We reproduce the list of generators, together with the action of each generator on $\pi_{1}\left(F_{g}\right)$ here. For an element $\psi \in \operatorname{MCG}^{\beta}\left(F_{g}\right)$, let $\psi_{\sharp}: \pi_{1}\left(F_{g}\right) \rightarrow \pi_{1}\left(F_{g}\right)$ be its action on $\pi_{1}\left(F_{g}\right)$. We let $a_{1}, b_{1}, \ldots, a_{g}, b_{g}$ be a set of standard generators of $\pi_{1}\left(F_{g}\right)$, with each $b_{i}$ contractible in the handlebody, and each $a_{i}$ intersecting $b_{i}$ once. Let $s_{i}=a_{i}^{-1} b_{i}^{-1} a_{i} b_{i}$, so that $s_{n} \cdots s_{2} s_{1}=1$ is a relation in $\pi_{1}\left(F_{g}\right)$. In [20], a genus $g$ surface is considered as a sphere with $g$ handles attached. Each handle, together with its immediate base, is called a knob. We refer to that paper for diagrams and geometric description of these generators.

Theorem 5.4 (Suzuki [20]) The group $\operatorname{MCG}^{\beta}\left(F_{g}\right)$ is generated by $\rho, \omega_{1}, \tau_{1}, \rho_{12}, \theta_{12}$ and $\xi_{12}$, whose actions on $\pi_{1}\left(F_{g}\right)$ are the following:

- Cyclic translation of handles $\rho_{\sharp}: a_{i} \rightarrow a_{i+1}, b_{i} \rightarrow b_{i+1}$, where indices are taken modulo $g$.

- Twisting a knob $\omega_{1 \sharp}: a_{1} \rightarrow a_{1}^{-1} s_{1}^{-1}, b_{1} \rightarrow a_{1}^{-1} b_{1}^{-1} a_{1}, a_{j} \rightarrow a_{j}, b_{j} \rightarrow b_{j}$ for $2 \leq j \leq n$.

- Twisting a handle, or Dehn twist $\tau_{1 \sharp}: a_{1} \rightarrow a_{1} b_{1}^{-1}, b_{1} \rightarrow b_{1}, a_{j} \rightarrow a_{j}$, $b_{j} \rightarrow b_{j}$ for $2 \leq j \leq n$.

- Interchanging two knobs $\rho_{12 \sharp}: a_{1} \rightarrow s_{1}^{-1} a_{2} s_{1}, a_{2} \rightarrow a_{1}, b_{1} \rightarrow s_{1}^{-1} b_{2} s_{1}$, $b_{2} \rightarrow b_{1}, a_{j} \rightarrow a_{j}, b_{j} \rightarrow b_{j}$ for $3 \leq j \leq n$.

- Sliding along $a_{2} \theta_{12 \sharp}: a_{1} \rightarrow a_{1}\left(b_{2}^{-1} a_{2}^{-1} b_{2}\right), a_{j} \rightarrow a_{j}$ for $j \neq 1, b_{2} \rightarrow$ $a_{2} b_{2}\left(a_{1}^{-1} b_{1} a_{1}\right)\left(b_{2}^{-1} a_{2}^{-1} b_{2}\right), b_{j} \rightarrow b_{j}$ for $j \neq 2$.

- Sliding along $\boldsymbol{b}_{2} \xi_{12 \sharp}: a_{1} \rightarrow b_{1} a_{1} b_{2}^{-1} s_{2}\left(a_{1}^{-1} b_{1}^{-1} a_{1}\right), a_{2} \rightarrow a_{2} b_{2}\left(a_{1}^{-1} b_{1}^{-1} a_{1}\right) b_{2}^{-1}$, $a_{j} \rightarrow a_{j}$ for $j \neq 1,2, b_{i} \rightarrow b_{i}$ for $1 \leq i \leq g$.

Of these, only $\rho$ is nonlocal in the sense that it is not restricted to a part of the surface with fixed genus. All other generators are restricted to a genus 1 or 2 part of the surface. We can remove $\rho$ in favor of other local generators by writing

$$
\rho^{-1}=\rho_{12} \circ \rho_{23} \circ \cdots \circ \rho_{g-1, g} \circ\left(\omega_{g \sharp}\right)^{-2},
$$

where $\omega_{g \sharp}$ is similar to $\omega_{1 \sharp}$, except acting on the $g^{\text {th }}$ handle, and $\rho_{i, i+1}$ interchanges the $i^{\text {th }}$ and $(i+1)^{\text {th }}$ knobs. The equation can be verified by comparing the actions of two sides on $\pi_{1}\left(F_{g}\right)$ : the initial $\left(\omega_{g \sharp}\right)^{-2}$ has the effect of conjugating $a_{g}$ and $b_{g}$ by $s_{g}^{-1}$. After interchanging the knobs in succession, the action of the right side on $\pi_{1}\left(F_{g}\right)$ is $a_{1} \rightarrow s_{1}^{-1} s_{2}^{-1} \cdots s_{g}^{-1} a_{g} s_{g} \cdots s_{2} s_{1}, a_{2} \rightarrow a_{1}, a_{3} \rightarrow a_{2}$, and so on, and similarly for the $b_{i}$ 's. We then apply the relation $s_{g} \cdots s_{2} s_{1}=1$. 
Since the boundary Dehn twist equals $\rho^{g}$, the $g^{\text {th }}$ power of the cyclic translation of handles, the same generators also generate the group $\operatorname{MCG}_{0}^{\beta}\left(\mathcal{Z}_{g}\right)$. Thus, we have proven the following.

Corollary 5.5 The group $\mathrm{MCG}_{0}^{\beta}\left(F_{g}\right)$ is generated by $\omega_{1}, \omega_{g}, \tau_{1}, \theta_{12}, \xi_{12}$ and $\rho_{i, i+1}$ for $1 \leq i \leq g-1$.

Each of the generators in Corollary 5.5 is confined to one or two knobs on the surface. Our strategy will be to check (27) on a surface of the corresponding genus, that is, 1 or 2 , then extend the result to the general case. First, we compute a decomposition of these generators into arcslides. An arcslide with $B$ pair $\left(b_{1}, b_{2}\right)$ and $C$ pair $\left(c_{1}, c_{2}\right)$, with $b_{1}$ sliding over $c_{1}$, is denoted $b_{1} \rightarrow c_{1}$. The points are always labeled 0 to $4 g-1$ from left to right. The results are

$$
\begin{aligned}
\rho_{12}: 3 & \rightarrow 4,6 \rightarrow 7,5 \rightarrow 6,4 \rightarrow 5,2 \rightarrow 3,5 \rightarrow 6,4 \rightarrow 5,3 \rightarrow 4, \\
1 & \rightarrow 2,4 \rightarrow 5,3 \rightarrow 4,2 \rightarrow 3,0 \rightarrow 1,3 \rightarrow 4,2 \rightarrow 3,1 \rightarrow 2, \\
\theta_{12}: 4 & \rightarrow 3,1 \rightarrow 0,1 \rightarrow 2,5 \rightarrow 4,6 \rightarrow 5, \\
\xi_{12}: & \rightarrow 1,3 \rightarrow 4,6 \rightarrow 7,6 \rightarrow 5,2 \rightarrow 3,1 \rightarrow 2,3 \rightarrow 2, \\
\omega_{1}: 2 & \rightarrow 3,1 \rightarrow 2,2 \rightarrow 3,1 \rightarrow 2,2 \rightarrow 3,1 \rightarrow 2, \\
\tau_{1}: 2 & \rightarrow 3 .
\end{aligned}
$$

To verify these decompositions, we compute their actions on $\pi_{1}\left(F^{\circ}\left(\mathcal{Z}^{g}\right)\right)$. For any pointed matched circle $\mathcal{Z}$, recall that the surface with circle boundary $F^{\circ}(\mathcal{Z})$ is formed by attaching 1-handles to $Z$ along the matched pairs of points in $\boldsymbol{a} \subset Z$, then gluing in a solid disk on the other side. Choosing $z \in Z$ as the basepoint, the fundamental group of $F^{\circ}(\mathcal{Z})$ is generated freely by paths through the 1 -handles. We choose the following orientation for the generators of the fundamental group. For the genus 1 cases, we have
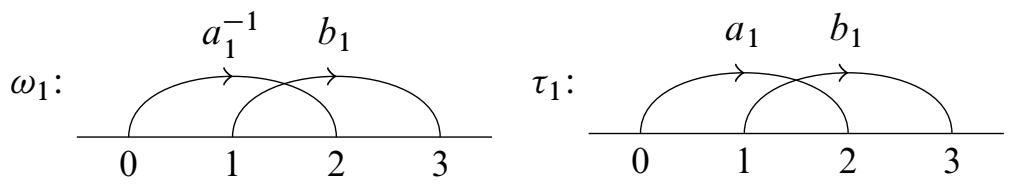

and for all genus 2 cases,

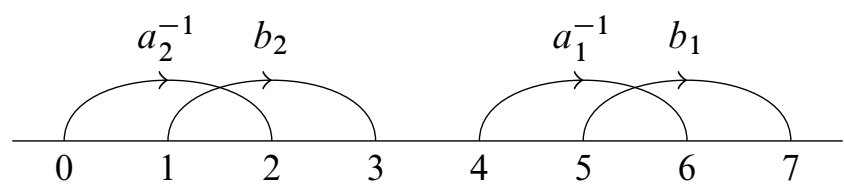




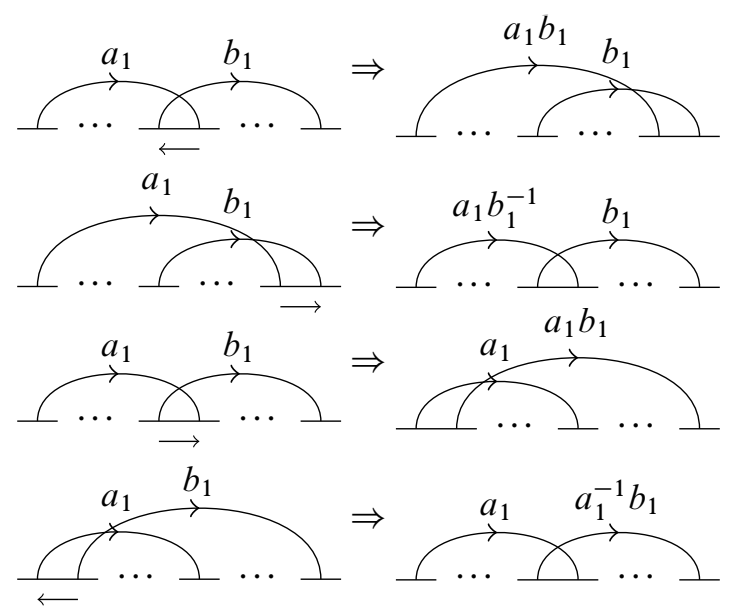

Figure 18: Actions of arcslides on the fundamental group (underslide)

An arcslide $\tau: \mathcal{Z}_{1} \rightarrow \mathcal{Z}_{2}$ induces an action

$$
\tau_{*}: \pi_{1}\left(F^{\circ}\left(\mathcal{Z}_{1}\right)\right) \rightarrow \pi_{1}\left(F^{\circ}\left(\mathcal{Z}_{2}\right)\right)
$$

on the fundamental groups. We describe this action by expressing each generator of $\pi_{1}\left(F^{\circ}\left(\mathcal{Z}_{2}\right)\right.$ ) (corresponding to a pair of matched points in $\left.\mathcal{Z}_{2}\right)$ in terms of the images under $\tau_{*}$ of generators of $\pi_{1}\left(F^{\circ}\left(\mathcal{Z}_{1}\right)\right)$. This can be computed from the definition of arcslides; see, for example, [11, Figure 3]. The results are shown in Figures 18 and 19. For example, the first diagram means that if the two displayed handles in the starting pointed matched circle correspond to generators $a_{1}$ and $b_{1}$, then the two displayed handles in the ending pointed matched circle correspond to $\tau_{*}\left(a_{1} b_{1}\right)$ and $\tau_{*}\left(b_{1}\right)$ (the relation for handles unaffected by the arcslide is clear).

As an example, we verify the decomposition of $\theta_{12}$ into arcslides. Only the two middle pairs, corresponding to generators $b_{2}$ and $a_{1}^{-1}$, are moved during this sequence of arcslides. We follow what happens to these two pairs in Table 1. We identify pairs of points in a sequence of arcslides as before. Each line in the table writes the generator corresponding to pairs identified with the initial $b_{2}$ and $a_{1}^{-1}$ pairs in terms of $\tau_{*}(\cdot)$ of the initial generators.

After this sequence of arcslides, the two middle pairs have switched positions. So the action is $a_{1}^{-1} \rightarrow b_{2}^{-1} a_{2} b_{2} a_{1}^{-1}$ and $b_{2} \rightarrow a_{2} b_{2} a_{1}^{-1} b_{1} a_{1} b_{2}^{-1} a_{2}^{-1} b_{2}$. The first equation can be rewritten as $a_{1} \rightarrow a_{1} b_{2}^{-1} a_{2}^{-1} b_{2}$. This agrees with the fundamental group action given in Theorem 5.4.

Proof of Lemma 5.3 The same argument as in the proof of Lemma 5.1 shows that if $\widehat{\mathrm{CFAD}}(\phi) \otimes \widehat{\mathrm{CFD}}\left(\boldsymbol{H}^{g}\right) \simeq \widehat{\mathrm{CFD}}\left(\boldsymbol{H}^{g}\right)$, then $\widehat{\mathrm{CFAD}}(\phi) \otimes \widehat{\mathrm{CFD}}\left(\boldsymbol{H}^{g+1}\right) \simeq \widehat{\mathrm{CFD}}\left(\boldsymbol{H}^{g+1}\right)$, 

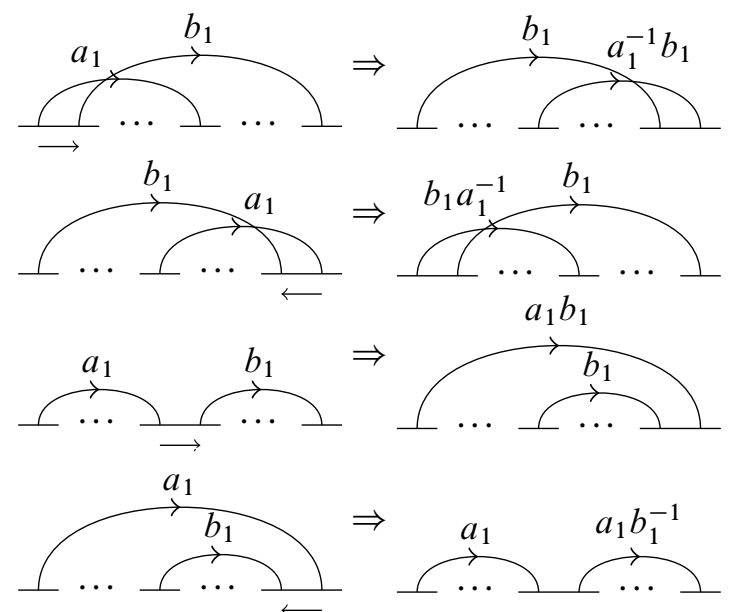

Figure 19: Actions of arcslides on the fundamental group (overslide)

\begin{tabular}{c|c|c} 
Arcslide & $b_{2}$ & $a_{1}^{-1}$ \\
\hline $4 \rightarrow 3$ & $b_{2}$ & $b_{2} a_{1}^{-1}$ \\
$1 \rightarrow 0$ & $b_{2}$ & $a_{2} b_{2} a_{1}^{-1}$ \\
$1 \rightarrow 2$ & $b_{2}^{-1} a_{2} b_{2} a_{1}^{-1}$ & $a_{2} b_{2} a_{1}^{-1}$ \\
$5 \rightarrow 4$ & $b_{2}^{-1} a_{2} b_{2} a_{1}^{-1}$ & $a_{2} b_{2} a_{1}^{-1} b_{1}$ \\
$6 \rightarrow 5$ & $b_{2}^{-1} a_{2} b_{2} a_{1}^{-1}$ & $a_{2} b_{2} a_{1}^{-1} b_{1} a_{1} b_{2}^{-1} a_{2}^{-1} b_{2}$
\end{tabular}

Table 1: Trajectories of generators $b_{2}$ and $a_{1}^{-1}$ under various arcslides.

where $\stackrel{\circ}{\phi}$ is the element of $\mathrm{MCG}_{0}\left(\mathcal{Z}^{g+1}\right)$ that fixes the new handle and acts as $\phi$ elsewhere. Here there is again an one-to-one correspondence on the generators between $\widehat{\mathrm{CFAD}}(\phi) \otimes \widehat{\mathrm{CFD}}\left(\boldsymbol{H}^{g}\right)$ and $\widehat{\mathrm{CFAD}}\left(\stackrel{\circ}{)} \otimes \widehat{\mathrm{CFD}}\left(\boldsymbol{H}^{g+1}\right)\right.$. There is exactly one domain in the adjoined portion that can (and does) contribute an arrow. The evaluation there is equivalent to the evaluation of $\widehat{\mathrm{CFAD}}\left(\mathbb{I}_{\mathcal{Z}}\right) \otimes \widehat{\mathrm{CFD}}\left(\boldsymbol{H}^{1}\right) \simeq \widehat{\mathrm{CFD}}\left(\boldsymbol{H}^{1}\right)$ on the genus 1 pointed matched circle, giving the arrow in $\widehat{\mathrm{CFD}}\left(\boldsymbol{H}^{g+1}\right)$ that is inside the adjoined pointed matched circle. The remaining domains must be outside the adjoined region, showing a one-to-one correspondence between arrows in $\widehat{\mathrm{CFAD}}(\phi) \otimes \widehat{\mathrm{CFD}}\left(\boldsymbol{H}^{g}\right)$, and

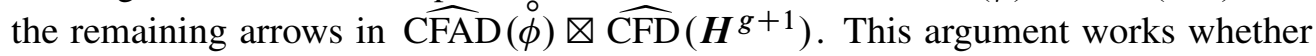
$\mathcal{Z}^{g+1}$ is formed as $\mathcal{Z}^{1} \# \mathcal{Z}^{g}$ or as $\mathcal{Z}^{g} \# \mathcal{Z}^{1}$.

From this, we see that it is sufficient to verify (27) for each of the generators of $\mathrm{MCG}_{0}^{\beta}\left(F_{g}\right)$ in its respective minimum genus (1 or 2$)$ case. 
To do so, we decompose each generator $\phi$ of $\mathrm{MCG}_{0}^{\beta}\left(F_{g}\right)$ (for $g=1$ or 2 depending on $\phi$ ) into arcslides $\tau_{n} \circ \cdots \circ \tau_{1}$, as given above. Then we directly compute the left side of (27) using the constructions for $\widehat{\mathcal{D A}}\left(\tau_{i}\right)$. This reduces to a finite computation, which we performed on a computer using a Python program (which implements the description of $\widehat{\mathcal{A A}}\left(\mathbb{I}_{\mathcal{Z}}\right)$ and the box tensor product). The code for the computation can be found at https://github.com/bzhan/auto2. The entire computation took less than 20 seconds.

This concludes the proof of Lemma 5.3, and therefore Theorem 2.4.

\section{References}

[1] J E Andersen, A J Bene, R C Penner, Groupoid extensions of mapping class representations for bordered surfaces, Topology Appl. 156 (2009) 2713-2725 MR2556030

[2] A J Bene, A chord diagrammatic presentation of the mapping class group of a once bordered surface, Geom. Dedicata 144 (2010) 171-190 MR2580425

[3] S Cautis, J Kamnitzer, Knot homology via derived categories of coherent sheaves, I: the sl(2)-case, Duke Math. J. 142 (2008) 511-588 MR2411561

[4] B Farb, D Margalit, A primer on mapping class groups, Princeton Mathematical Series 49, Princeton Univ. Press (2012) MR2850125

[5] M Khovanov, A functor-valued invariant of tangles, Algebr. Geom. Topol. 2 (2002) 665-741 MR1928174

[6] M Khovanov, P Seidel, Quivers, Floer cohomology, and braid group actions, J. Amer. Math. Soc. 15 (2002) 203-271 MR1862802

[7] M Khovanov, R Thomas, Braid cobordisms, triangulated categories, and flag varieties, Homology Homotopy Appl. 9 (2007) 19-94 MR2366943

[8] A S Levine, Knot doubling operators and bordered Heegaard Floer homology, J. Topol. 5 (2012) 651-712 MR2971610

[9] R Lipshitz, P S Ozsváth, D P Thurston, A faithful linear-categorical action of the mapping class group of a surface with boundary, J. Eur. Math. Soc. 15 (2013) 12791307 MR3055762

[10] R Lipshitz, P Ozsváth, D P Thurston, Bordered Heegaard Floer homology: invariance and pairing, preprint (2014) arXiv:0810.0687 To appear in J. Topol.

[11] R Lipshitz, PS Ozsváth, D P Thurston, Computing $\widehat{H F}$ by factoring mapping classes, Geom. Topol. 18 (2014) 2547-2681 MR3285222

[12] R Lipshitz, PS Ozsváth, DP Thurston, Bimodules in bordered Heegaard Floer homology, Geom. Topol. 19 (2015) 525-724 MR3336273 
[13] P Ozsváth, A I Stipsicz, Z Szabó, Combinatorial Heegaard Floer homology and nice Heegaard diagrams, Adv. Math. 231 (2012) 102-171 MR2935385

[14] P Ozsváth, Z Szabó, Holomorphic disks and three-manifold invariants: properties and applications, Ann. of Math. 159 (2004) 1159-1245 MR2113020

[15] P Ozsváth, Z Szabó, Holomorphic disks and topological invariants for closed threemanifolds, Ann. of Math. 159 (2004) 1027-1158 MR2113019

[16] S Sarkar, J Wang, An algorithm for computing some Heegaard Floer homologies, Ann. of Math. 171 (2010) 1213-1236 MR2630063

[17] P Seidel, I Smith, A link invariant from the symplectic geometry of nilpotent slices, Duke Math. J. 134 (2006) 453-514 MR2254624

[18] P Seidel, R Thomas, Braid group actions on derived categories of coherent sheaves, Duke Math. J. 108 (2001) 37-108 MR1831820

[19] K Siegel, A geometric proof of a faithful linear-categorical surface mapping class group action (2011) arXiv:1108.3676

[20] S Suzuki, On homeomorphisms of a 3-dimensional handlebody, Canad. J. Math. 29 (1977) 111-124 MR0433433

[21] B Zhan, Explicit Koszul-dualizing bimodules in bordered Heegaard Floer homology, Algebr. Geom. Topol. 16 (2016) 231-266 MR3470701

Department of Mathematics, Massachusetts Institute of Technology

Room 2-231B, 77 Massachusetts Avenue, Cambridge, MA 02139, United States

bzhan@mit.edu

Received: 5 July 2014 Revised: 9 January 2016 